\title{
Supporting Information \\ Synthesis of Dibenzofurans by \\ Cu-catalyzed Deborylative Ring Contraction of Dibenzoxaborins
}
Yuto Sumida, ${ }^{*}, \dagger, \|$ Ryu Harada,${ }^{\ddagger}$ Tomoe Sumida,${ }^{\dagger}$ Kohei Johmoto,${ }^{\S}$ Hidehiro Uekusa,${ }^{\S}$ and Takamitsu Hosoya ${ }^{* \dagger, \dagger}$
${ }^{\dagger}$ Laboratory for Chemical Biology, RIKEN Center for Biosystems Dynamics Research (BDR),
6-7-3 Minatojima-minamimachi, Chuo-ku, Kobe 650-0047, Japan
Laboratory of Chemical Bioscience, Institute of Biomaterials and Bioengineering,
Tokyo Medical and Dental University (TMDU),
2-3-10 Kanda-Surugadai, Chiyoda-ku, Tokyo 101-0062, Japan
${ }^{\S}$ Department of Chemistry, School of Science, Tokyo Institute of Technology,
2-12-1, Ookayama, Meguro-ku, Tokyo, 152-8551, Japan

"Present Address: Division of Pharmaceutical Sciences, Graduate School of Medical Sciences, Kanazawa University, Kakuma-machi, Kanazawa 920-1192, Japan

\section{Contents}

General Remarks $\quad$ S2

Detailed Results for Optimization of Reaction Conditions (Table S1) S4

Experimental Procedures $\quad$ S6

$\begin{array}{lr}\text { Characterization Data } & \text { S14 }\end{array}$

References for Supporting Information $\quad$ S27

$\begin{array}{lr}\text { Crystallographic Analyses } & \mathbf{S 2 8}\end{array}$

${ }^{1} \mathrm{H},{ }^{13} \mathrm{C}$, and ${ }^{19} \mathrm{~F}$ NMR Spectra of Compounds $\quad$ S37 


\section{General Remarks}

All manipulations of air- and/or moisture-sensitive compounds were performed in a glovebox UN$500 \mathrm{M}$ (UNICO) under an atmosphere of argon. All deborylative ring contractions were conducted in a $5 \mathrm{~mL}$ screw top V-vials (Wheaton) with a cap assembled with a septum (Wheaton). Aluminum heating block was used for all reactions that require heating, unless otherwise noted.

Analytical thin-layer chromatography (TLC) was performed on precoated $(0.25 \mathrm{~mm})$ silica-gel plates (Wako Pure Chemical Co., Wakogel ${ }^{\circledR}$ B5-F, or Merck, Merck Silica Gel $60 \mathrm{~F}_{254}$, Cat. No. 1.05715.0001). Preparative TLC was carried out using precoated silica-gel plates (Wako Pure Chemical Industries, Ltd., Wakogel ${ }^{\circledR}$ B5-F, Cat. No. 230-00043, or 0.50 mm: Merck, Merck Silica Gel $60 \mathrm{~F}_{254}$, Cat. No. 1.05744.0001. 1.0 mm: Merck, Merck Silica Gel $60 \mathrm{~F}_{254}$, Cat. No. 1.13895.0001. $2.0 \mathrm{~mm}$ : Merck, Merck Silica Gel $60 \mathrm{~F}_{254}$, Cat. No. 1.05717.0001). Column chromatography was conducted by hand using silica-gel (Kanto Chemical Co., Inc., Silica Gel 60N, Cat. No. 37563-84, Silica Gel 60, Cat. No. 37562-85, or SiliCycle Inc., SiliaFlash, Cat. No. R10030B) or Biotage ZIP ${ }^{\circledR}$ sphere cartridge [silica] $5 \mathrm{~g}$ (Cat. No. 445-0500-DZ-20), $10 \mathrm{~g}$ (Cat. No. 445-1000-EZ-20), $30 \mathrm{~g}$ (Cat. No. 445-3000-FZ-20) or 80 g (Cat. No. 445-8000-JZ-20) with medium pressure liquid chromatography (Yamazen, W-Prep 2XY A-type or AI-580 and Parallel Frac FR-360). Recycling preparative HPLC (High performance liquid chromatography) was performed by LC-908-C60 system (Japan Analytical Industry Co., Ltd.) with high resolution GPC column (Japan Analytical Industry Co., Ltd., JAIGEL-1H or YMC Co., Ltd., YMC-GPC T2000).

Melting points (mp) were measured with a Micro Melting Point System MP-J3 (Yanaco New Science Inc.) or an OptiMelt automated melting point apparatus (Stanford Research Systems, Inc.) and were uncorrected.

${ }^{1} \mathrm{H} \mathrm{NMR}(500$ or $400 \mathrm{MHz})$ and ${ }^{13} \mathrm{C} \mathrm{NMR}(126$ or $100 \mathrm{MHz})$ spectra obtained from measurements at ambient temperature were taken on a Bruker AVANCE 500 spectrometer, a Bruker AVANCE 400 spectrometer, or a JEOL 400SS spectrometer. ${ }^{19} \mathrm{~F}$ NMR $(372.5 \mathrm{MHz})$ spectra were obtained with a JEOL 400SS spectrometer. ${ }^{11} \mathrm{~B}$ NMR $(128$ or $127 \mathrm{MHz})$ spectra were obtained with a Bruker AVANCE 400 spectrometer or a JEOL 400SS spectrometer. Chloroform- $d_{1}\left(\mathrm{CDCl}_{3}\right)$ containing $0.03 \%$ tetramethylsilane (TMS) $(>99.8 \% \mathrm{D}$, Cambridge Isotope Laboratories, Inc., Cat. No. DLM-7 or Acros Organics, Cat. No. 368651000), $\mathrm{CD}_{3} \mathrm{OD}$ (Cambridge Isotope Laboratories, Inc., Cat. No. DLM-24), and acetone- $d_{6}(99.96 \% \mathrm{D}$, Cambridge Isotope Laboratories, Inc., Cat. No. DLM-38) were used as solvents for NMR measurements at ambient temperature. Chemical shifts $(\delta)$ for ${ }^{1} \mathrm{H}$ NMR are given in parts per million (ppm) relative to relative to TMS $\left(\delta 0.00 \mathrm{ppm}\right.$ in $\left.\mathrm{CDCl}_{3}\right)$, or residual acetone $(\delta 2.07 \mathrm{ppm})$. Chemical shifts $(\delta)$ for ${ }^{13} \mathrm{C} \mathrm{NMR}$ are given in ppm relative to $\mathrm{CDCl}_{3}(\delta 77.0$ $\mathrm{ppm})$, or residual acetone $(\delta 30.6 \mathrm{ppm})$. Chemical shifts $(\delta)$ for ${ }^{19} \mathrm{~F}$ NMR are given in ppm relative to $\alpha, \alpha, \alpha$-trifluorotoluene $\left(\delta-63.0 \mathrm{ppm}\right.$ in $\left.\mathrm{CDCl}_{3}\right)$ used as the external standard. Chemical shifts $(\delta)$ for ${ }^{11} \mathrm{~B} \mathrm{NMR}$ are given in ppm relative to $\mathrm{BF}_{3} \cdot \mathrm{OEt}_{2}\left(\delta 0.0 \mathrm{ppm}\right.$ in $\left.\mathrm{CDCl}_{3}\right)$ used as the external standard. The abbreviations s, d, t, q, m, and br signify singlet, doublet, triplet, quartet, multiplet, and broad, respectively.

IR spectra were measured by diffuse reflectance method or single reflection ATR method on a Shimadzu IRPrestige-21 spectrometer attached with DRS-8000A or MIRacle, or a Thermo Scientific Nicolet iS5 FT-IR attached with iD7 ATR with the absorption band given in $\mathrm{cm}^{-1}$.

Elemental analyses were carried out at the Elemental Analysis Center of Tokyo Institute of Technology or A Rabbit Science Japan Co., Ltd.

High-resolution mass spectra (HRMS) were measured on a JEOL JMS-T100LC AccuTOF mass spectrometer under electron ionization (EI) conditions, a JEOL JMS-700V under negative electrospray ionization $\left(\mathrm{ESI}^{-}\right)$conditions at Molecular Characterization, Collaboration Promotion Unit, RIKEN, or a Thermo Scientific Exactive Plus Orbitrap under positive $\left(\mathrm{ESI}^{+}\right)$conditions. 
Intensity data of X-ray crystallographical analyses were collected on a Rigaku R-AXIS RAPID II diffractometer. The structure was solved by direct methods (SHELXS-2013) and refined by the fullmatrix least-squares on $F^{2}$ (SHELXL-2013). CCDC 2003738 (compound 1q) contains the supplementary crystallographic data for this structure. This data can be obtained free of charge from the Cambridge Crystallographic Data Centre (CCDC) via www.ccdc.cam.ac.uk/data_request/cif.

All chemical reagents used were commercial grade and used as received unless otherwise noted.

Trifluoromethanesulfonic anhydride was kindly provided from Central Glass Co., Ltd.

Starting materials 1a-1o, ${ }^{\mathrm{S} 1} \mathbf{1 p},{ }^{\mathrm{S} 2} \mathbf{5},{ }^{\mathrm{S} 3} \mathbf{6},{ }^{\mathrm{S} 1} \mathbf{9},{ }^{\mathrm{S} 3}$ and $\mathbf{S 1 a}-\mathbf{S 1} \mathbf{c}^{\mathrm{S} 1}$ were prepared in conventional ways according to the literature.<smiles>OB1Oc2ccccc2-c2ccccc21</smiles>

$1 \mathrm{a}$<smiles>OB1Oc2ccc(C(F)(F)F)cc2-c2ccccc21</smiles>

1d<smiles>OB1Oc2c(-c3ccccc3)cccc2-c2ccccc21</smiles>

$1 \mathrm{~g}$<smiles>Cc1ccc2c(c1)-c1cc(F)ccc1B(O)O2</smiles><smiles>OB1Oc2ccccc2-c2ncccc21</smiles>

10<smiles>O=[N+]([O-])c1ccc2c(c1)-c1ccccc1B(O)O2</smiles>

1e<smiles>Cc1ccc2c(c1)-c1ccccc1OB2O</smiles><smiles>COC(=O)c1ccc2c(c1)-c1ccccc1B(O)O2</smiles><smiles>OB1Oc2cc(C(F)(F)F)ccc2-c2ccccc21</smiles>

$1 f$<smiles>COc1ccc2c(c1)-c1ccccc1OB2O</smiles><smiles>COc1cc(C)cc2c1-c1cc(OC)c3c(OCc4ccccc4)cccc3c1OB2O</smiles><smiles>OB1CCc2ccccc2O1</smiles><smiles></smiles>

5<smiles>COC(=O)c1ccc(O)c(Br)c1</smiles>

S1a<smiles>CCCc1ccccc1OCc1ccccc1</smiles>

6<smiles>COc1cc(C)cc(Br)c1O[Ga]</smiles>

S1b<smiles>CCOc1cc2c(cc1B=O)C1CC[C@]3(C)[C@@H]([OH+])CC[C@H]3[C@@H]1CC2</smiles>

9

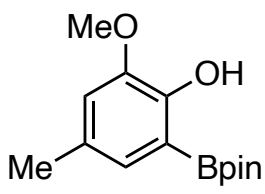

S1c 
Table S1. Detailed Results for Optimization of Reaction Conditions

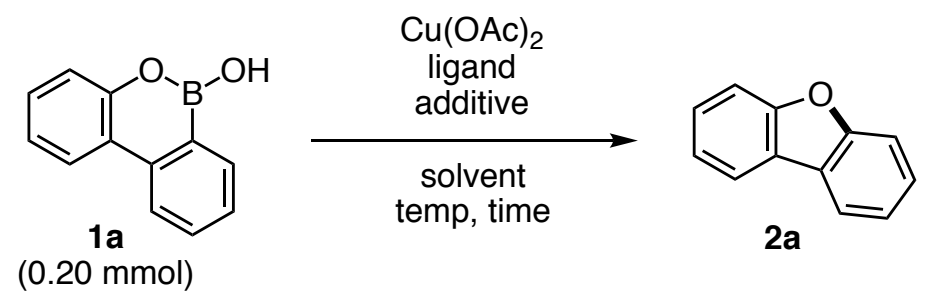

\begin{tabular}{|c|c|c|c|c|c|c|c|}
\hline entry & $\begin{array}{c}\text { equiv of } \\
\mathrm{Cu}(\mathrm{OAc})_{2}\end{array}$ & ligand (equiv) & additive (equiv) & slovent & $\begin{array}{l}\text { temp } \\
\left({ }^{\circ} \mathrm{C}\right)\end{array}$ & $\begin{array}{l}\text { time } \\
(\mathrm{h})\end{array}$ & $\begin{array}{l}\text { yield } \\
(\%)^{a}\end{array}$ \\
\hline 1 & 0.10 & none & none & $\mathrm{MeOH}$ & 40 & 40 & 20 \\
\hline $2^{b}$ & 0.10 & 1,10-phen $(0.12)$ & $\mathrm{BzOOBz}(2.5)$ & 1,2-DCE & 60 & 20 & 32 \\
\hline $3^{b}$ & 0.10 & 1,10-phen $(0.12)$ & $t$-BuOOBz (2.5) & 1,2-DCE & 80 & 20 & 77 \\
\hline 4 & 0.10 & 1,10 -phen $(0.12)$ & $i$ - $\operatorname{Pr}_{2} \mathrm{NEt}(1.5)$ & 1,2-DCE & 60 & 20 & 51 \\
\hline 5 & 0.10 & 1,10 -phen $(0.12)$ & $i$ - $\operatorname{Pr}_{2} \mathrm{NEt}(1.5)$ & 1,2-DCE & $\mathrm{rt}$ & 28 & 43 \\
\hline 6 & 0.10 & bpy $(0.12)$ & $i-\mathrm{Pr}_{2} \mathrm{NEt}(1.5)$ & 1,2-DCE & $\mathrm{rt}$ & 20 & 33 \\
\hline 7 & 0.10 & dtbpy (0.12) & $i-\operatorname{Pr}_{2} \mathrm{NEt}(1.5)$ & 1,2-DCE & $\mathrm{rt}$ & 20 & 36 \\
\hline 8 & 0.10 & 1,10-phen $(0.12)$ & $i$ - $\operatorname{Pr}_{2} \operatorname{NEt}(1.5)$ & $\mathrm{MeOH}$ & $\mathrm{rt}$ & 28 & 50 \\
\hline 9 & 0.10 & 1,10 -phen $(0.12)$ & $i-\operatorname{Pr}_{2} \mathrm{NEt}(1.5)$ & $\mathrm{EtOH}$ & $\mathrm{rt}$ & 20 & 52 \\
\hline 10 & 0.10 & 1,10 -phen $(0.12)$ & $\mathrm{Et}_{3} \mathrm{~N}(1.5)$ & $\mathrm{EtOH}$ & $\mathrm{rt}$ & 20 & 52 \\
\hline 11 & 0.10 & 1,10-phen $(0.12)$ & $\mathrm{Cs}_{2} \mathrm{CO}_{3}(1.5)$ & 1,2-DCE & 60 & 20 & 15 \\
\hline 12 & 0.10 & 1,10 -phen $(0.12)$ & $\mathrm{Cs}_{2} \mathrm{CO}_{3}(1.5)$ & $\mathrm{EtOH}$ & 40 & 19 & 56 \\
\hline 13 & 0.10 & 1,10 -phen $(0.12)$ & $\mathrm{K}_{2} \mathrm{CO}_{3}(1.5)$ & $\mathrm{EtOH}$ & 40 & 19 & 21 \\
\hline 14 & 0.10 & 1,10 -phen $(0.12)$ & $t$-BuOK (1.5) & $\mathrm{EtOH}$ & 40 & 19 & 9 \\
\hline 15 & 0.10 & 1,10-phen $(0.12)$ & $\mathrm{MeOK}(1.5)$ & $\mathrm{EtOH}$ & 40 & 19 & 52 \\
\hline 16 & 0.10 & 1,10-phen $(0.12)$ & $\mathrm{Ag}_{2} \mathrm{CO}_{3}(1.5)$ & dehyd. EtOH & 40 & 16 & 71 \\
\hline 17 & 0.10 & 1,10-phen $(0.12)$ & $\mathrm{Ag}_{2} \mathrm{CO}_{3}(1.5)$ & $\mathrm{EtOH} / \mathrm{H}_{2} \mathrm{O}(100 / 1)$ & 40 & 10 & 77 \\
\hline 18 & 0.10 & 1,10-phen $(0.12)$ & $\mathrm{Ag}_{2} \mathrm{CO}_{3}(1.5)$ & $\mathrm{EtOH} / \mathrm{H}_{2} \mathrm{O}(20 / 1)$ & 40 & 19 & $\begin{array}{l}\text { quant } \\
(95)^{c}\end{array}$ \\
\hline 19 & 0.10 & 1,10-phen $(0.12)$ & $\mathrm{Ag}_{2} \mathrm{CO}_{3}(1.5)$ & $\mathrm{EtOH} / \mathrm{H}_{2} \mathrm{O}(10 / 1)$ & 40 & 10 & 97 \\
\hline 20 & 0.10 & 1,10-phen (0.12) & $\mathrm{Ag}_{2} \mathrm{CO}_{3}(1.5)$ & $\mathrm{EtOH} / \mathrm{H}_{2} \mathrm{O}(5 / 1)$ & 40 & 10 & 86 \\
\hline 21 & 0.10 & neocuproine $(0.12)$ & $\mathrm{Ag}_{2} \mathrm{CO}_{3}(1.0)$ & $\mathrm{EtOH} / \mathrm{H}_{2} \mathrm{O}(20 / 1)$ & 40 & 21 & 62 \\
\hline 22 & 0.10 & 1,10 -phen $(0.12)$ & $\mathrm{Ag}_{2} \mathrm{CO}_{3}(1.5)$ & $\mathrm{EtOH} / \mathrm{H}_{2} \mathrm{O}(20 / 1)$ & $\mathrm{rt}$ & 46 & $\begin{array}{l}\text { quant } \\
(96)^{c}\end{array}$ \\
\hline
\end{tabular}




\begin{tabular}{cccccccc}
\hline 23 & 0.050 & 1,10 -phen (0.06) & $\mathrm{Ag}_{2} \mathrm{CO}_{3}(1.5)$ & $\mathrm{EtOH} / \mathrm{H}_{2} \mathrm{O}(20 / 1)$ & 40 & 30 & 86 \\
$24^{d}$ & 0.10 & 1,10 -phen (0.12) & $\mathrm{Ag}_{2} \mathrm{CO}_{3}(1.5)$ & $\mathrm{EtOH} / \mathrm{H}_{2} \mathrm{O}(20 / 1)$ & 40 & 16 & 89 \\
25 & 0.10 & 1,10 -phen (0.12) & $\mathrm{Ag}_{2} \mathrm{CO}_{3}(0.15)$ & $\mathrm{EtOH} / \mathrm{H}_{2} \mathrm{O}(20 / 1)$ & 40 & 16 & 51 \\
$26^{b}$ & 0.10 & 1,10 -phen (0.12) & none & $\mathrm{EtOH} / \mathrm{H}_{2} \mathrm{O}(20 / 1)$ & 40 & 16 & 8 \\
27 & 1.0 & 1,10 -phen (1.1) & none & $\mathrm{EtOH} / \mathrm{H}_{2} \mathrm{O}(20 / 1)$ & 40 & 16 & 39 \\
28 & none & 1,10 -phen (1.1) & $\mathrm{Ag}_{2} \mathrm{CO}_{3}(1.0)$ & $\mathrm{EtOH} / \mathrm{H}_{2} \mathrm{O}(20 / 1)$ & 40 & 16 & 5 \\
29 & 0.10 & 1,10 -phen (0.12) & $\mathrm{AgOAc}^{2}(1.5)$ & $\mathrm{EtOH} / \mathrm{H}_{2} \mathrm{O}(20 / 1)$ & 40 & 19 & 21 \\
30 & 0.10 & 1,10 -phen (0.12) & $\mathrm{AgNO}_{3}(1.5)$ & $\mathrm{EtOH} / \mathrm{H}_{2} \mathrm{O}(20 / 1)$ & 40 & 19 & 4 \\
31 & 0.10 & 1,10 -phen (0.12) & $\mathrm{Ag}_{3} \mathrm{PO}_{4}(1.5)$ & $\mathrm{EtOH} / \mathrm{H}_{2} \mathrm{O}(20 / 1)$ & 40 & 16 & 0 \\
32 & 0.10 & 1,10 -phen (0.12) & $\mathrm{Ag}_{2} \mathrm{O}(1.5)$ & $\mathrm{EtOH} / \mathrm{H}_{2} \mathrm{O}(20 / 1)$ & 40 & 16 & 93 \\
$33^{b}$ & 0.10 & 1,10 -phen (0.12) & $\mathrm{Ag}_{2} \mathrm{O}(1.5)$ & $\mathrm{EtOH} / \mathrm{H}_{2} \mathrm{O}(20 / 1)$ & 40 & 19 & 93 \\
34 & 0.10 & 1,10 -phen (0.12) & $\mathrm{Ag}_{2} \mathrm{O}(1.5)$ & $\mathrm{EtOH} / \mathrm{H}_{2} \mathrm{O}(20 / 1)$ & $\mathrm{rt}$ & 37 & $86^{c}$ \\
\hline
\end{tabular}

${ }^{a}$ Yields were determined by ${ }^{1} \mathrm{H}$ NMR analysis unless otherwise noted. ${ }^{b}$ The reaction was performed under argon. ${ }^{c}$ Isolated yields shown in parentheses. ${ }^{d}$ The reaction was performed in degassed $\mathrm{EtOH} / \mathrm{H}_{2} \mathrm{O}$ under argon. 


\section{Experimental Procedures}

A typical procedure for copper-catalyzed deborylative ring contraction of dibenzoxaborin<smiles>OB1Oc2ccccc2-c2ccccc21</smiles>

$1 a$

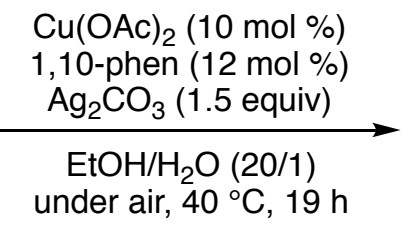

under air, $40^{\circ} \mathrm{C}, 19 \mathrm{~h}$<smiles>c1ccc2c(c1)oc1ccccc12</smiles>

2a

To a mixture of copper(II) acetate (3.6 mg, $20 \mu \mathrm{mol})$, 1,10-phenanthroline ( $4.3 \mathrm{mg}, 24 \mu \mathrm{mol})$, and silver(I) carbonate $(82.7 \mathrm{mg}, 0.300 \mathrm{mmol})$ in ethanol $(2.0 \mathrm{~mL})$ and water $(0.10 \mathrm{~mL})$ was added dibenzoxaborin (1a) $(39.2 \mathrm{mg}, 0.200 \mathrm{mmol})$ at room temperature. After stirring for $19 \mathrm{~h}$ at $40{ }^{\circ} \mathrm{C}$ under air, the mixture was cooled to room temperature and filtered through a cotton-plugged funnel using $n$-hexane $(10 \mathrm{~mL})$. The filtrate was concentrated under reduced pressure and the residue was purified by silica-gel column chromatography ( $n$-hexane only) to give dibenzofuran (2a) (31.8 $\mathrm{mg}$, $0.189 \mathrm{mmol}, 94.5 \%$ ) as a colorless solid.

\section{A gram-scale experiment}

To a mixture of copper(II) acetate (92.6 mg, $510 \mu \mathrm{mol}), 1,10$-phenanthroline (114 mg, $630 \mu \mathrm{mol})$, and silver(I) carbonate $(1.74 \mathrm{~g}, 6.30 \mathrm{mmol})$ in ethanol $(51 \mathrm{~mL})$ and water $(2.6 \mathrm{~mL})$ was added dibenzoxaborin (1a) $(1.00 \mathrm{~g}, 5.10 \mathrm{mmol})$ at room temperature. After stirring for $19 \mathrm{~h}$ at $40{ }^{\circ} \mathrm{C}$ under air, the mixture was cooled to room temperature and filtered through a cotton-plugged funnel with $n$ hexane $(50 \mathrm{~mL})$. The filtrate was concentrated under reduced pressure and the residue was purified by silica-gel column chromatography ( $n$-hexane only) to give dibenzofuran (2a) (736 mg, $4.38 \mathrm{mmol}$, $85.8 \%$ ) as a colorless solid.

Stoichiometric reaction in the presence of $\mathrm{Ag}_{2} \mathrm{CO}_{3}$ (Figure 4A)

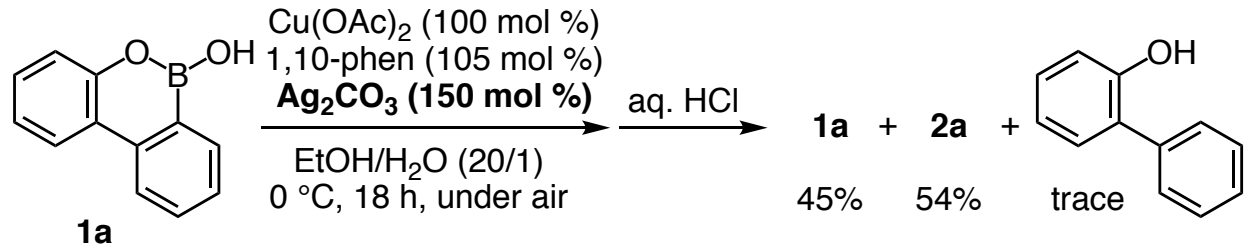

To a mixture of copper(II) acetate $(36.3 \mathrm{mg}, 0.200 \mathrm{mmol}), 1,10$-phenanthroline $(43.2 \mathrm{mg}, 0.210$ $\mathrm{mmol})$, and silver(I) carbonate $(82.7 \mathrm{mg}, 0.300 \mathrm{mmol})$ in ethanol $(2.0 \mathrm{~mL})$ and water $(0.10 \mathrm{~mL})$ was added dibenzoxaborin (1a) $(39.2 \mathrm{mg}, 0.200 \mathrm{mmol})$ at $0{ }^{\circ} \mathrm{C}$. After stirring for $18 \mathrm{~h}$ at the same temperature under air, to the mixture was added aqueous $\mathrm{HCl}(1.0 \mathrm{M}, 2 \mathrm{~mL})$ and extracted with $n$ hexane/EtOAc $(5 / 1,2 \mathrm{~mL} \times 3)$. The combined organic extract was concentrated under reduced pressure and to the residue was added 1,1,2,2-tetrachloroethane $(20 \mu \mathrm{L})$ as an internal standard. The mixture was dissolved in $\mathrm{CDCl}_{3}$, and then ${ }^{1} \mathrm{H}$ NMR was measured. The ${ }^{1} \mathrm{H}$ NMR yields of 1a, 2a, and 2-phenylphenol were obtained by comparing the relative value of integration for the peaks of protons for 1a $(8.07 \mathrm{ppm}, 1 \mathrm{H}), \mathbf{2 a}(7.96 \mathrm{ppm}, 2 \mathrm{H})$, and 2-phenylphenol (6.95-7.04 ppm, 2H), respectively, with those of 1,1,2,2-tetrachloroethane observed at $5.91 \mathrm{ppm}(2 \mathrm{H})$. 
Stoichiometric reaction without $\mathrm{Ag}_{2} \mathrm{CO}_{3}$ (Figure 4B)

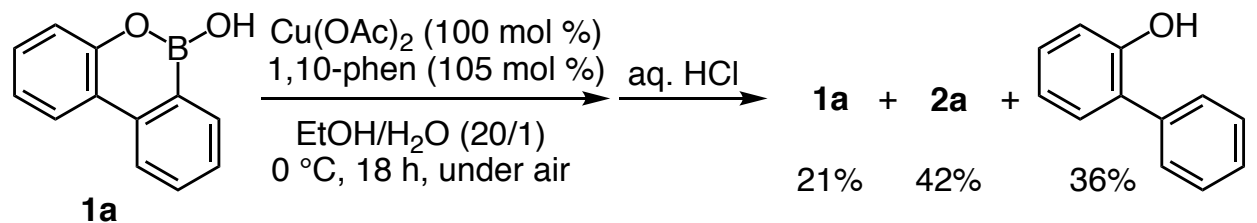

To a mixture of copper(II) acetate $(36.3 \mathrm{mg}, 0.200 \mathrm{mmol})$ and 1,10-phenanthroline $(43.2 \mathrm{mg}, 0.210$ $\mathrm{mmol})$ in ethanol $(2.0 \mathrm{~mL})$ and water $(0.10 \mathrm{~mL})$ was added dibenzoxaborin (1a) $(39.2 \mathrm{mg}, 0.200$ $\mathrm{mmol}$ ) at $0{ }^{\circ} \mathrm{C}$. After stirring for $18 \mathrm{~h}$ at the same temperature under air, to the mixture was added aqueous $\mathrm{HCl}(1.0 \mathrm{M}, 2 \mathrm{~mL})$ and extracted with $n$-hexane/EtOAc $(5 / 1,2 \mathrm{~mL} \times 3)$. The combined organic extract was concentrated under reduced pressure and to the residue was added 1,1,2,2tetrachloroethane $(20 \mu \mathrm{L})$ as an internal standard. The mixture was dissolved in $\mathrm{CDCl}_{3}$, and then ${ }^{1} \mathrm{H}$ NMR was measured. The ${ }^{1} \mathrm{H}$ NMR yields of 1a, 2a, and 2-phenylphenol were obtained by comparing the relative value of integration for the peaks of protons for $\mathbf{1 a}(8.07 \mathrm{ppm}, 1 \mathrm{H}), \mathbf{2 a}(7.96 \mathrm{ppm}, 2 \mathrm{H})$, and 2-phenylphenol (6.95-7.04 ppm, 2H), respectively, with those of 1,1,2,2-tetrachloroethane observed at $5.91 \mathrm{ppm}(2 \mathrm{H})$.

The analyses of reaction stoichiometry I (Figure 5A)

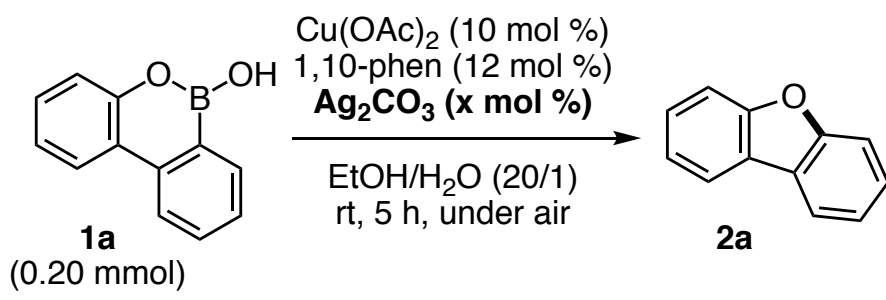

To a mixture of copper(II) acetate $(3.6 \mathrm{mg}, 20 \mu \mathrm{mol})$, 1,10-phenanthroline ( $4.3 \mathrm{mg}, 24 \mu \mathrm{mol})$, and silver(I) carbonate $(\mathrm{x} \mathrm{mmol})$ in ethanol $(2.0 \mathrm{~mL})$ and water $(0.10 \mathrm{~mL})$ was added dibenzoxaborin $1 \mathrm{a}$ (39.2 $\mathrm{mg}, 0.200 \mathrm{mmol}$ ) at room temperature. After stirring for $5 \mathrm{~h}$ at the same temperature under air, the mixture was filtered through a cotton-plugged funnel using $n$-hexane $(10 \mathrm{~mL})$ and concentrated under reduced pressure. To the residue was added 1,1,2,2-tetrachloroethane $(20 \mu \mathrm{L})$ as an internal standard. The mixture was dissolved in $\mathrm{CDCl}_{3}$, and then ${ }^{1} \mathrm{H}$ NMR was measured. The ${ }^{1} \mathrm{H}$ NMR yields of dibenzofuran (2a) were obtained by comparing the relative value of integration for the peaks of protons for $2 \mathrm{a}(7.96 \mathrm{ppm}, 2 \mathrm{H})$ with those of 1,1,2,2-tetrachloroethane observed at $5.91 \mathrm{ppm}(2 \mathrm{H})$. The results are shown below in Table S2.

Table S2

\begin{tabular}{cccc}
\hline entry & $\mathrm{Ag}_{2} \mathrm{CO}_{3}(\mathrm{mmol})$ & $\mathrm{Ag}_{2} \mathrm{CO}_{3}(\mathrm{x} \mathrm{mol} \mathrm{\% )}$ & ${ }^{1} \mathrm{H}$ NMR yield of 2a $(\%)$ \\
\hline 1 & 0.050 & 25 & 30.4 \\
2 & 0.100 & 50 & 52.3 \\
3 & 0.200 & 100 & 58.8 \\
4 & 0.300 & 150 & 54.2 \\
5 & 0.400 & 200 & 56.2 \\
6 & 0.500 & 250 & 59.5 \\
\hline
\end{tabular}


The analyses of reaction stoichiometry II (Figure 5B)

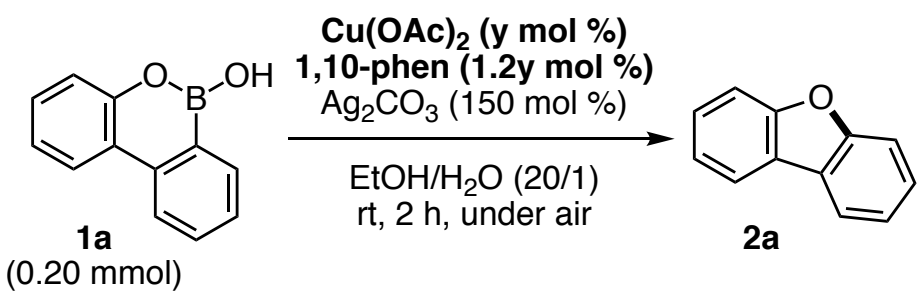

To a mixture of copper(II) acetate (y mmol), 1,10-phenanthroline (1.2y mmol), and silver(I) carbonate $(82.7 \mathrm{mg}, 0.300 \mathrm{mmol})$ in ethanol $(2.0 \mathrm{~mL})$ and water $(0.10 \mathrm{~mL})$ was added dibenzoxaborin $1 \mathrm{a}(39.2 \mathrm{mg}, 0.200 \mathrm{mmol})$ at room temperature. After stirring for $2 \mathrm{~h}$ at the same temperature under air, the mixture was filtered through a cotton-plugged funnel using $n$-hexane $(10 \mathrm{~mL})$ and concentrated under reduced pressure. To the residue was added 1,1,2,2-tetrachloroethane $(20 \mu \mathrm{L})$ as an internal standard. The mixture was dissolved in $\mathrm{CDCl}_{3}$, and then ${ }^{1} \mathrm{H}$ NMR was measured. The ${ }^{1} \mathrm{H}$ NMR yields of dibenzofuran (2a) were obtained by comparing the relative value of integration for the peaks of protons for $\mathbf{2 a}(7.96 \mathrm{ppm}, 2 \mathrm{H})$ with those of 1,1,2,2-tetrachloroethane observed at 5.91 ppm $(2 \mathrm{H})$. The results are shown below in Table S3.

Table S3

\begin{tabular}{cccc}
\hline entry & $\begin{array}{c}\mathrm{Cu}(\mathrm{OAc})_{2} / 1,10-\text { phen } \\
(\mathrm{mmol})\end{array}$ & $\begin{array}{c}\mathrm{Cu}(\mathrm{OAc})_{2} \\
(\mathrm{y} \mathrm{mol} \mathrm{\% )}\end{array}$ & ${ }^{1} \mathrm{H}$ NMR yield of 2a $(\%)$ \\
\hline 1 & $0.020 / 0.024$ & 10 & 22.2 \\
2 & $0.040 / 0.048$ & 20 & 40.2 \\
3 & $0.080 / 0.096$ & 40 & 55.9 \\
4 & $0.160 / 0.192$ & 80 & 61.2 \\
5 & $0.320 / 0.384$ & 160 & 71.8 \\
6 & $0.640 / 0.768$ & 320 & 78.3 \\
\hline
\end{tabular}


The analyses of reaction stoichiometry III

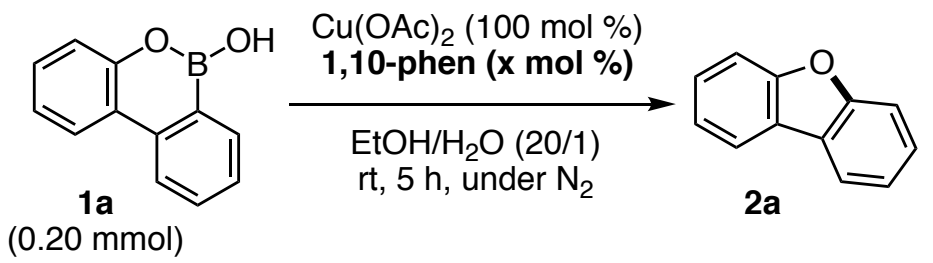

To a mixture of copper(II) acetate $(0.20 \mathrm{mmol})$ and 1,10 -phenanthroline (x mmol) in ethanol $(2.0$ $\mathrm{mL})$ and water $(0.10 \mathrm{~mL})$ was added dibenzoxaborin $1 \mathbf{a}(39.2 \mathrm{mg}, 0.200 \mathrm{mmol})$ at room temperature. After stirring for $5 \mathrm{~h}$ at the same temperature under $\mathrm{N}_{2}$ the mixture was filtered through a cottonplugged funnel using $n$-hexane $(10 \mathrm{~mL})$ and concentrated under reduced pressure. The mixture was dissolved in $\mathrm{CDCl}_{3}$, and then ${ }^{1} \mathrm{H}$ NMR was measured. The ${ }^{1} \mathrm{H}$ NMR yields of remaining dibenzoxaborin 1a were obtained by comparing the relative value of integration for the peaks of protons for $1 \mathrm{a}(7.71 \mathrm{ppm}, 1 \mathrm{H})$ with those of 1,1,2,2-tetrachloroethane observed at $5.91 \mathrm{ppm}(2 \mathrm{H})$. The ${ }^{1} \mathrm{H}$ NMR yields of dibenzofuran (2a) were obtained by comparing the relative value of integration for the peaks of protons for $\mathbf{2 a}(7.96 \mathrm{ppm}, 2 \mathrm{H})$ with those of 1,1,2,2-tetrachloroethane observed at $5.91 \mathrm{ppm}(2 \mathrm{H})$. The results are shown below in Table $\mathrm{S} 4$.

Table S4

\begin{tabular}{ccccc}
\hline entry & 1,10-phen $(\mathrm{x} \mathrm{mol} \mathrm{\% )}$ & $\begin{array}{c}{ }^{1} \mathrm{H} \text { NMR yield } \\
\text { of 1a }(\%)\end{array}$ & $\begin{array}{c}{ }^{1} \mathrm{H} \text { NMR yield } \\
\text { of 2a }(\%)\end{array}$ & ratio of 1a/2a \\
\hline 1 & 120 & 69 & 28 & $71 / 29$ \\
2 & 10 & 83 & 6 & $93 / 7$ \\
3 & 0 & 95 & 2 & $98 / 2$ \\
\hline
\end{tabular}

Preparation of dibenzoxaborin 11 by boron-selective Suzuki-Miyaura coupling

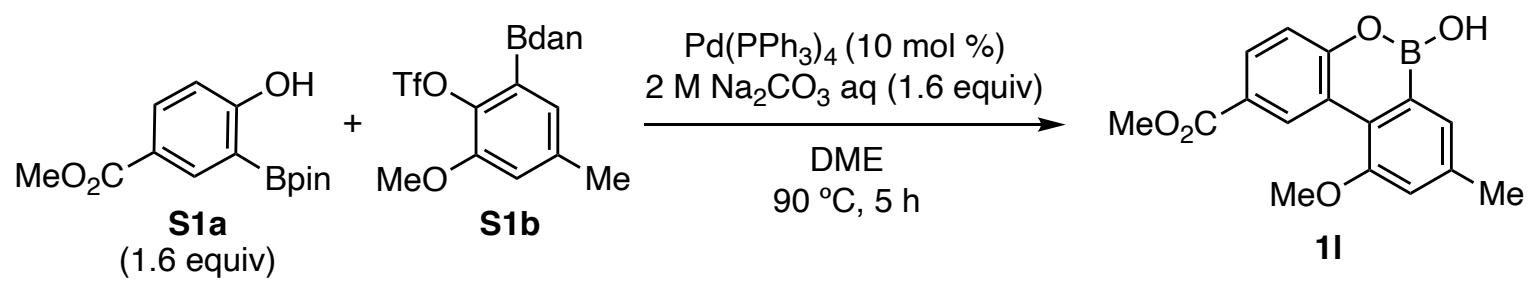

To a solution of $\mathbf{S 1 a} \mathbf{a}^{\mathrm{S} 1}(44.5 \mathrm{mg}, 0.160 \mathrm{mmol}), \mathbf{S 1} \mathbf{b}^{\mathrm{S} 1}(43.6 \mathrm{mg}, 0.100 \mathrm{mmol})$, and $\mathrm{Pd}\left(\mathrm{PPh}_{3}\right)_{4}(11.6$ $\mathrm{mg}, 10.0 \mu \mathrm{mol})$ in 1,2-dimethoxyethane $(1 \mathrm{~mL})$ was added aqueous $\mathrm{Na}_{2} \mathrm{CO}_{3}(2 \mathrm{M}, 80 \mu \mathrm{L}, 0.16 \mathrm{mmol})$ at room temperature. After stirring with heating at $90{ }^{\circ} \mathrm{C}$ for $5 \mathrm{~h}$, the mixture was cooled to room temperature and then concentrated under reduced pressure. The residue was purified by preparative TLC ( $n$-hexane/EtOAc/MeOH $=80 / 20 / 1)$ to give 6-hydroxy-10-methoxy-2-methoxycarbonyl-8methyl-6H-dibenzo[c,e][1,2]oxaborin (11) $(13.2 \mathrm{mg}, 44.3 \mu \mathrm{mol}, 44.3 \%)$ as a colorless solid. 
Preparation of dibenzoxaborin 1 m by boron-selective Suzuki-Miyaura coupling<smiles>COc1cc(C)cc(Br)c1O</smiles>

(1.5 equiv)<smiles>COc1cc(C)cc(Br)c1O</smiles>

S1b

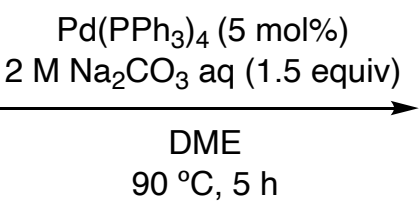

$90^{\circ} \mathrm{C}, 5 \mathrm{~h}$<smiles>COc1cc(C)cc2c1OB(O)c1cc(C)cc(OC)c1-2</smiles>

To a solution of $\mathbf{S 1} \mathrm{c}^{\mathrm{S} 1}(79.2 \mathrm{mg}, 0.300 \mathrm{mmol}), \mathbf{S 1}{ }^{\mathrm{S} 1}(87.2 \mathrm{mg}, 0.200 \mathrm{mmol})$, and $\mathrm{Pd}\left(\mathrm{PPh}_{3}\right)_{4}(11.6$ $\mathrm{mg}, 10.0 \mu \mathrm{mol})$ in 1,2-dimethoxyethane $(2 \mathrm{~mL})$ was added aqueous $\mathrm{Na}_{2} \mathrm{CO}_{3}(2 \mathrm{M}, 150 \mu \mathrm{L}, 0.30$ mmol) at room temperature. After stirring with heating at $90{ }^{\circ} \mathrm{C}$ for $5 \mathrm{~h}$, the mixture was cooled to room temperature and then concentrated under reduced pressure. The residue was purified by preparative TLC ( $n$-hexane/EtOAc/MeOH $=80 / 20 / 1)$ to give 4,10-dimethoxy-2,8-dimethyl-6Hdibenzo[c,e][1,2] oxaborin (1m) $(35.2 \mathrm{mg}, 0.124 \mathrm{mmol}, 61.9 \%)$ as a colorless solid.

Preparation of $1 \mathbf{q}$ by bromination of dibenzoxaborin $1 \mathbf{a}$

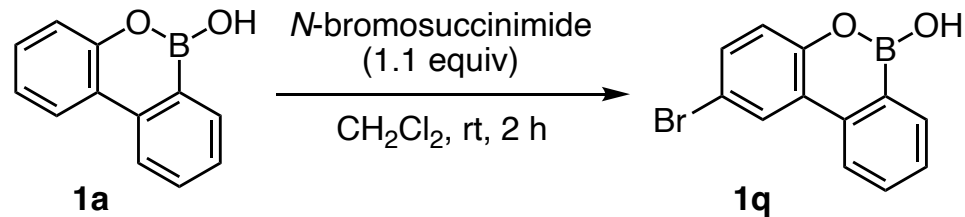

To a solution of $1 \mathrm{a}(98.0 \mathrm{mg}, 0.500 \mathrm{mmol})$ in dichloromethane $(10 \mathrm{~mL})$ was added $\mathrm{N}$ bromosuccinimide $(101 \mathrm{mg}, 0.567 \mathrm{mmol})$ at room temperature. After stirring for $2 \mathrm{~h}$ at the same temperature under air, to the mixture was added an aqueous saturated solution of sodium thiosulfate and the mixture was extracted with EtOAc $(10 \mathrm{~mL} \times 3)$. The combined organic extract was washed with brine $(10 \mathrm{~mL})$, dried over $\mathrm{Na}_{2} \mathrm{SO}_{4}$, and after filtration, the filtrate was concentrated under reduced pressure. The residue was purified by preparative TLC ( $n$-hexane/EtOAc/MeOH $=90 / 10 / 1$ ) to give 2-bromo-6-hydroxy-6H-dibenzo[c,e][1,2]oxaborin (1q) $(118 \mathrm{mg}, 0.429 \mathrm{mmol}, 85.9 \%)$ as a colorless solid. A part of 1q was recrystallized from EtOAc to obtain a sample for X-ray crystallographic analyses.

Preparation of $1 \boldsymbol{r}$ by iodination of dibenzoxaborin 1 a

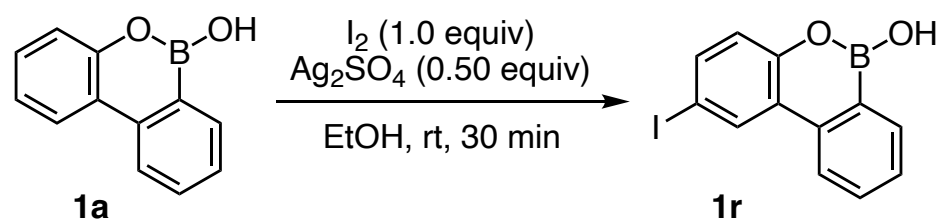

To a mixture of $1 \mathrm{a}(1.96 \mathrm{~g}, 10.0 \mathrm{mmol})$ and silver sulfate $(1.56 \mathrm{~g}, 5.00 \mathrm{mmol})$ in ethanol $(40 \mathrm{~mL})$ was slowly added a solution of iodine $(2.54 \mathrm{~g}, 10.0 \mathrm{mmol})$ in ethanol $(40 \mathrm{~mL})$ over $30 \mathrm{~min}$ at room temperature. After filtration of the mixture through a short pad of celite, to the filtrate was added a saturated aqueous solution of sodium thiosulfate and the mixture was extracted with EtOAc (20 $\mathrm{mL} \times 3)$. The combined organic extract was washed with brine $(10 \mathrm{~mL})$, dried over $\mathrm{Na}_{2} \mathrm{SO}_{4}$, and after filtration, the filtrate was concentrated under reduced pressure. The residue was purified by silica-gel column chromatography $(n$-hexane/EtOAc/ $\mathrm{MeOH}=90 / 10 / 1)$ to give 6-hydroxy-2-iodo- $6 H$-dibenzo$[c, e][1,2]$ oxaborin (1r) (1.43 g, $4.44 \mathrm{mmol}, 44.4 \%)$ as a colorless solid. 
Preparation of 1 s by Migita-Kosugi-Stille coupling of dibenzoxaborin 1q with stannane 3<smiles>CCCCc1cccs1</smiles>

$$
\begin{gathered}
\mathrm{Pd}(\mathrm{OAc})_{2}(5.0 \mathrm{~mol} \%) \\
\mathrm{SPhos}(10 \mathrm{~mol} \%) \\
\mathrm{K}_{3} \mathrm{PO}_{4} \cdot \mathrm{nH}_{2} \mathrm{O}(1.5 \text { equiv }) \\
\underset{\text { toluene, } 65^{\circ} \mathrm{C}, 18 \mathrm{~h}}{\longrightarrow}
\end{gathered}
$$

(1.1 equiv)<smiles>OB1Oc2ccc(-c3cccs3)cc2-c2ccccc21</smiles>

$1 \mathrm{~s}$

To a mixture of $\mathbf{1 q}(137 \mathrm{mg}, 0.498 \mathrm{mmol})$, palladium acetate $(5.6 \mathrm{mg}, 25 \mu \mathrm{mol})$, 2dicyclohexylphosphino-2',6'-dimethoxybiphenyl (SPhos) ( $20.5 \mathrm{mg}, 50.0 \mu \mathrm{mol}$ ), and $\mathrm{K}_{3} \mathrm{PO}_{4} \cdot n \mathrm{H}_{2} \mathrm{O}$ $(80 \%, 199 \mathrm{mg}, 0.750 \mathrm{mmol})$ in toluene (dehydrated, $10 \mathrm{~mL}$ ) was added tributyl(thiophen-2yl)stannane (3) $(185 \mu \mathrm{L}, 0.555 \mathrm{mmol})$ at room temperature under argon, and the resulting mixture was heated at $65{ }^{\circ} \mathrm{C}$ with oil bath for $18 \mathrm{~h}$. After cooling to room temperature, to this was added a saturated aqueous solution of ammonium chloride and the mixture was extracted with EtOAc (5 $\mathrm{mL} \times 3)$. The combined organic extract was washed with brine $(2 \mathrm{~mL})$, dried over $\mathrm{Na}_{2} \mathrm{SO}_{4}$, and after filtration, the filtrate was concentrated under reduced pressure. The residue was purified by preparative TLC ( $n$-hexane/EtOAc $=5 / 1)$ to give 6-hydroxy-2-(thiophen-2-yl)-6Hdibenzo[c,e][1,2]oxaborin (1s) $(122 \mathrm{mg}, 0.439 \mathrm{mmol}, 88.1 \%)$ as a pale gray solid.

Preparation of $\mathbf{1 t}$ by Sonogashira coupling of dibenzoxaborin $1 \mathbf{r}$ with alkyne 4

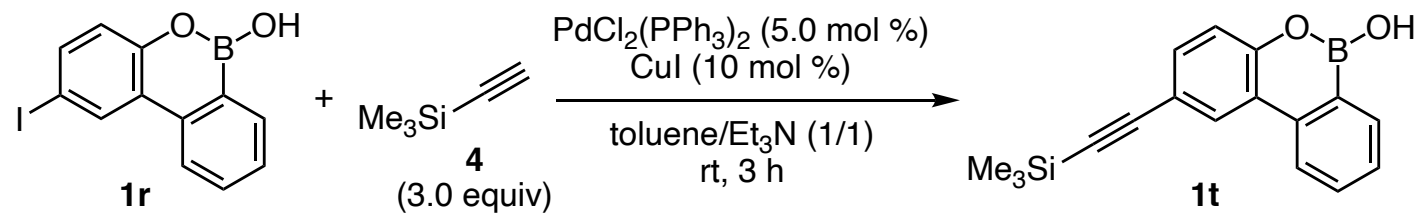

To a mixture of $1 \mathbf{r}$ (322 mg, $1.00 \mathrm{mmol}$ ), dichlorobis(triphenylphosphine)palladium (35.0 mg, 50.0 $\mu \mathrm{mol})$, and copper iodide $(19.0 \mathrm{mg}, 0.100 \mathrm{mmol})$ in toluene (dehydrated, $2.5 \mathrm{~mL}$ ) and triethyl amine $(2.5 \mathrm{~mL})$ was added trimethylsilylacetylene $(4)(420 \mu \mathrm{L}, 2.97 \mathrm{mmol})$ at room temperature under argon. After stirring for $3 \mathrm{~h}$ at the same temperature, to this was added a saturated aqueous solution of ammonium chloride and the mixture was extracted with EtOAc $(5 \mathrm{~mL} \times 3)$. The combined organic extract was washed with brine $(2 \mathrm{~mL})$, dried over $\mathrm{Na}_{2} \mathrm{SO}_{4}$, and after filtration, the filtrate was concentrated under reduced pressure. To the residue was added EtOAc $(1 \mathrm{~mL})$ and $\mathrm{MeOH}(1 \mathrm{~mL})$, and then the resulting insoluble solid was collected by filtration. The filtrate was concentrated under reduced pressure and the same operation was repeated three times. The combined solid was washed with a small amount of $\mathrm{MeOH}$ on a Kiriyama funnel and dried under reduced pressure to give 6hydroxy-2-[(trimethylsilyl)ethynyl]-6H-dibenzo[c,e][1,2]oxaborin (1t) (252 mg, $0.862 \mathrm{mmol}$, $86.2 \%)$ as a pale gray solid. 
Preparation of $\mathbf{1} \boldsymbol{u}$ by boron-selective Suzuki-Miyaura coupling of $\mathbf{5}$ with $\mathbf{6}$
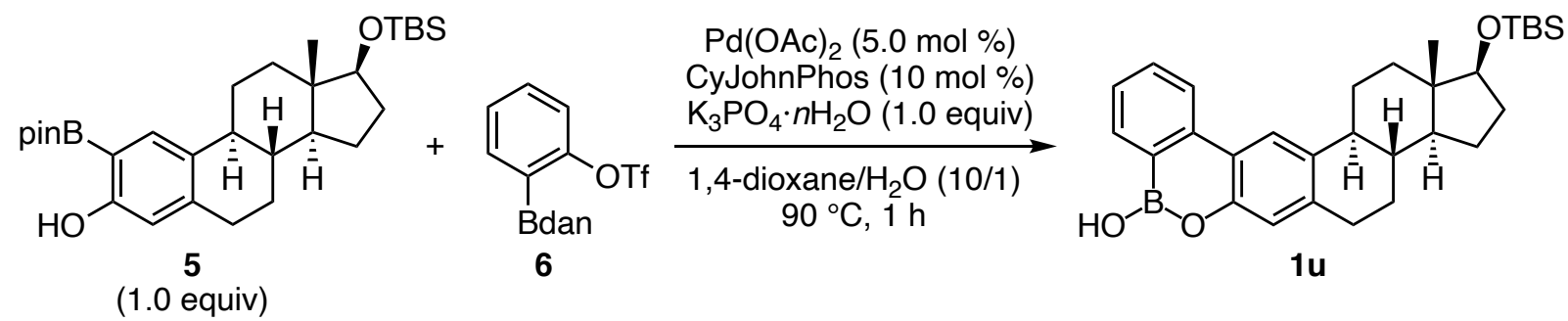

To a mixture of $\mathbf{5}^{\mathrm{S} 3}(1.03 \mathrm{~g}, 2.01 \mathrm{mmol})$, palladium acetate $(22.5 \mathrm{mg}, 100 \mu \mathrm{mol})$, 2dicyclohexylphosphino-1,1'-biphenyl (CyJohnPhos) $(70.1 \mathrm{mg}, 200 \mu \mathrm{mol})$, and $\mathrm{K}_{3} \mathrm{PO}_{4} \cdot n \mathrm{H}_{2} \mathrm{O}(80 \%$, $530 \mathrm{mg}, 2.00 \mathrm{mmol})$ in 1,4-dioxane $(20 \mathrm{~mL})$ and distilled water $(2.0 \mathrm{~mL})$ was added $6^{\mathrm{S} 1}(784 \mathrm{mg}$, $2.00 \mathrm{mmol}$ ) at room temperature under argon, and the resulting mixture was heated at $90{ }^{\circ} \mathrm{C}$ with oil bath for $1 \mathrm{~h}$. After cooling to room temperature, to this was added $\mathrm{AcOH}(2 \mathrm{~mL})$ and water $(20 \mathrm{~mL})$, and the mixture was extracted with $n$-hexane/EtOAc $(5 / 1,20 \mathrm{~mL} \times 3)$. The combined organic extract was washed with brine $(20 \mathrm{~mL})$, dried over $\mathrm{Na}_{2} \mathrm{SO}_{4}$, and after filtration, the filtrate was concentrated under reduced pressure. The residue was purified by silica-gel column chromatography $(n-$ hexane/EtOAc $=5 / 1)$ to give $1 \mathbf{u}(405 \mathrm{mg}, 0.829 \mathrm{mmol}, 41.4 \%)$ as a colorless solid.

Preparation of 7 from 5 by triflylation and protecting group exchange from Bpin to Bdan ${ }^{S 4}$

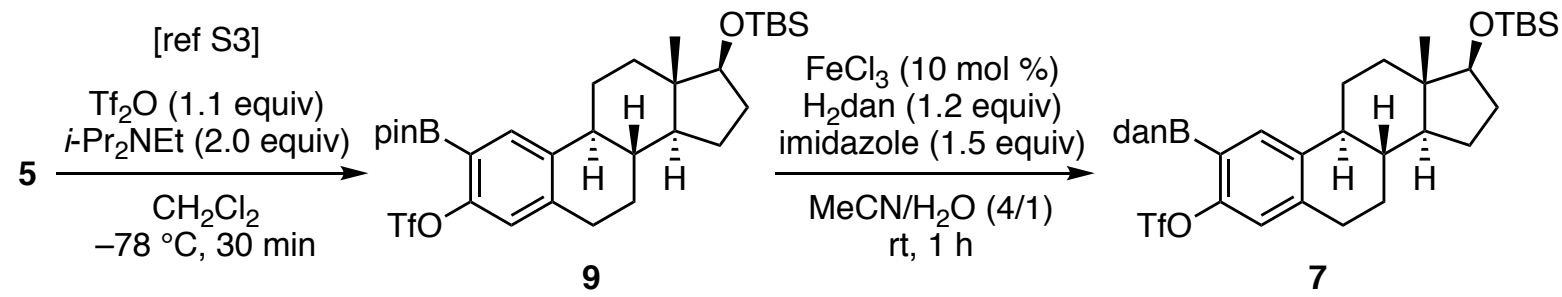

To a mixture of $9(967 \mathrm{mg}, 1.50 \mathrm{mmol}$, prepared in $93.0 \%$ yield from 5 according to the previous report $\left.{ }^{\mathrm{S} 3}\right), 1,8$-diaminonaphthalene $\left(\mathrm{H}_{2} \mathrm{dan}\right)(285 \mathrm{mg}, 1.80 \mathrm{mmol})$, and imidazole $(153 \mathrm{mg}, 2.25 \mathrm{mmol})$ in $\mathrm{MeCN}(24 \mathrm{~mL})$ and distilled water $(6.0 \mathrm{~mL})$ was added $\mathrm{FeCl}_{3}(24.3 \mathrm{mg}, 0.150 \mathrm{mmol})$ at room temperature. After stirring for $1 \mathrm{~h}$ at the same temperature, to this was added water $(20 \mathrm{~mL})$ and the mixture was extracted with EtOAc $(20 \mathrm{~mL} \times 3)$. The combined organic extract was concentrated under reduced pressure and the residue was purified by silica-gel column chromatography and preparative TLC $(n$-hexane/EtOAc $=5 / 1)$ to give $7(885 \mathrm{mg}, 1.29 \mathrm{mmol}, 80.2 \%$ in two steps from 5) as a pale gray solid.

Preparation of $1 \mathbf{v}$ by boron-selective Suzuki-Miyaura coupling of 7 with $\mathbf{8}$

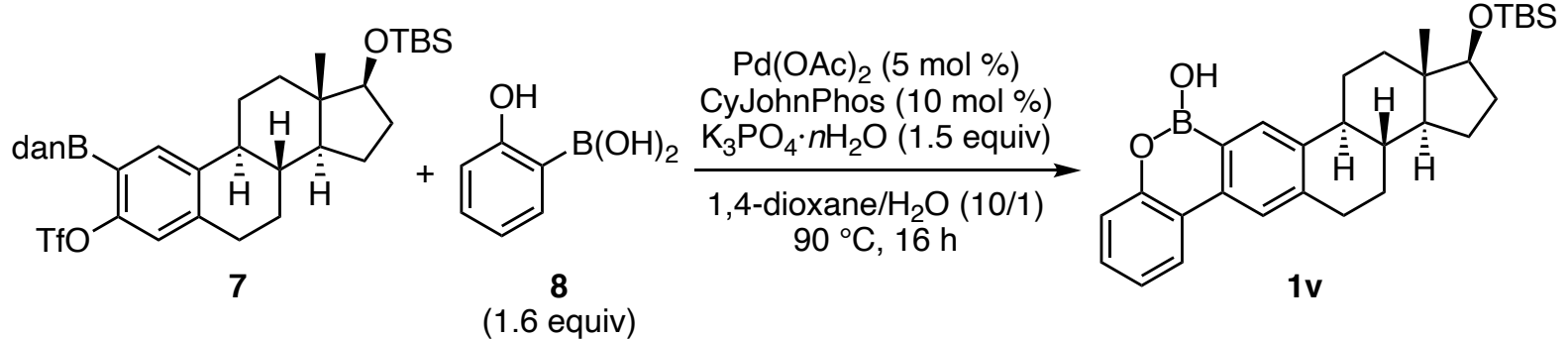

To a solution of 7 (324 mg, $0.473 \mathrm{mmol})$, palladium acetate $(5.60 \mathrm{mg}, 25.0 \mu \mathrm{mol})$, 2dicyclohexylphosphino-1,1'-biphenyl (CyJohnPhos) $(17.5 \mathrm{mg}, 50.0 \mu \mathrm{mol})$ and $\mathrm{K}_{3} \mathrm{PO}_{4} \cdot n \mathrm{H}_{2} \mathrm{O}(80 \%$, 
$199 \mathrm{mg}, 0.750 \mathrm{mmol})$ and in 1,4-dioxane $(4.0 \mathrm{~mL})$ distilled water $(0.40 \mathrm{~mL})$ was added (2hydroxyphenyl)boronic acid (8) $(103 \mathrm{mg}, 0.747 \mathrm{mmol})$ at room temperature under argon, and the resulting mixture was heated at $90{ }^{\circ} \mathrm{C}$ for $16 \mathrm{~h}$. After cooling to room temperature, to this was added phosphate buffer $(1.0 \mathrm{M}, \mathrm{pH} 7.4,2 \mathrm{~mL})$ and water $(2 \mathrm{~mL})$, and the mixture was extracted with EtOAc $(4 \mathrm{~mL} \times 3)$. The combined organic extract was washed with brine $(2 \mathrm{~mL})$, dried $\left(\mathrm{Na}_{2} \mathrm{SO}_{4}\right)$, and after filtration, the filtrate was concentrated under reduced pressure. The residue was purified by silica-gel column chromatography ( $n$-hexane/EtOAc $=5 / 1$ ) and recycling preparative HPLC using a high resolution GPC column (eluent: $\left.\mathrm{CHCl}_{3}\right)$ to give $1 \mathbf{v}(67.5 \mathrm{mg}, 0.138 \mathrm{mmol}, 29.2 \%)$ as a pale gray solid. 


\section{Characterization Data}

6-Hydroxy-10-methoxy-2-methoxycarbonyl-8-methyl-6H-dibenzo[c,e][1,2]oxaborin (11)<smiles>COc1cc(C)cc2c1-c1cc(C(C)=O)ccc1OB2O</smiles>

Colorless solid;

mp $253-255^{\circ} \mathrm{C}$;

TLC $R_{\mathrm{f}} 0.30(n$-hexane/EtOAc $=4 / 1)$;

${ }^{1} \mathrm{H}$ NMR $\left(\mathrm{CDCl}_{3}\right.$ and 1 drop of $\left.\mathrm{CD}_{3} \mathrm{OD}, 400 \mathrm{MHz}\right): \delta 9.80(\mathrm{~s}, 1 \mathrm{H}), 8.00(\mathrm{~d}, J=8.4 \mathrm{~Hz}, 1 \mathrm{H}), 7.49(\mathrm{~s}$, $1 \mathrm{H}), 7.33(\mathrm{~d}, J=8.4 \mathrm{~Hz}, 1 \mathrm{H}), 7.07(\mathrm{~s}, 1 \mathrm{H}), 4.06(\mathrm{~s}, 3 \mathrm{H}), 3.95(\mathrm{~s}, 3 \mathrm{H}), 2.46(\mathrm{~s}, 3 \mathrm{H})($ the signal for the proton of boronic acid was not observed);

${ }^{13} \mathrm{C}\left\{{ }^{1} \mathrm{H}\right\} \mathrm{NMR}\left(\mathrm{CDCl}_{3}, 100 \mathrm{MHz}\right): \delta 167.4,157.5,154.3,138.7,131.5,128.9,125.4,125.3,124.1$, $122.8,119.1,116.2,55.6,52.0,21.5$ (the signal for the carbon attached to the boron atom was not observed);

${ }^{11} \mathrm{~B} \mathrm{NMR}\left(\mathrm{CDCl}_{3}, 128 \mathrm{MHz}\right): \delta 27.5$;

IR $\left(\mathrm{cm}^{-1}\right)$ 3362, 2952, 1689, 1599, 1514, 1435, 1412, 1372, 1272, 1165, 1138, 1100, 1065, 896, 821, 770, 731, 698, 668;

HRMS $\left(\mathrm{ESI}^{+}\right) \mathrm{m} / z$ : $[\mathrm{M}+\mathrm{Na}]^{+}$calcd for $\mathrm{C}_{16} \mathrm{H}_{15} \mathrm{BO}_{\mathrm{Na}}{ }^{+}, 321.0905$; found, 321.0900 .

\section{4,10-Dimethoxy-2,8-dimethyl-6H-dibenzo $[c, e][1,2]$ oxaborin $(1 \mathrm{~m})$}<smiles>COc1cc(C)cc2c1OB(O)c1cc(C)cc(OC)c1-2</smiles>

Colorless solid;

mp $143-145^{\circ} \mathrm{C}$;

TLC $R_{\mathrm{f}} 0.45(n$-hexane/EtOAc $=4 / 1)$;

${ }^{1} \mathrm{H} \mathrm{NMR}\left(\mathrm{CDCl}_{3}, 400 \mathrm{MHz}\right): \delta 8.48(\mathrm{~s}, 1 \mathrm{H}), 7.55(\mathrm{~s}, 1 \mathrm{H}), 7.07(\mathrm{~s}, 1 \mathrm{H}), 6.80(\mathrm{~s}, 1 \mathrm{H}), 4.88(\mathrm{~s}, 1 \mathrm{H})$, $4.01(\mathrm{~s}, 3 \mathrm{H}), 3.97(\mathrm{~s}, 3 \mathrm{H}), 2.46(\mathrm{~s}, 3 \mathrm{H}), 2.44(\mathrm{~s}, 3 \mathrm{H})$;

${ }^{13} \mathrm{C}\left\{{ }^{1} \mathrm{H}\right\} \mathrm{NMR}\left(\mathrm{CDCl}_{3}, 100 \mathrm{MHz}\right): \delta 157.5,148.5,138.5,138.2,130.8,126.6,125.9,123.3,121.1$, $116.8,111.4,56.2,55.6,22.0,21.4$ (the signal for the carbon attached to the boron atom was not observed);

${ }^{11} \mathrm{~B} \mathrm{NMR}\left(\mathrm{CDCl}_{3}, 128 \mathrm{MHz}\right): \delta 28.3$;

IR $\left(\mathrm{cm}^{-1}\right)$ 3444, 2914, 2846, 1605, 1588, 1448, 1383, 1365, 1348, 1313, 1286, 1265, 1194, 1161 , $1134,1029,841,830,777,719,667,602$;

HRMS $\left(\mathrm{ESI}^{+}\right) \mathrm{m} / z:[\mathrm{M}+\mathrm{Na}]^{+}$calcd for $\mathrm{C}_{16} \mathrm{H}_{17} \mathrm{BO}_{4} \mathrm{Na}^{+}$, 307.1112; found, 307.1106.

\section{Dibenzofuran (2a)}<smiles>c1ccc2c(c1)oc1ccccc12</smiles>

Yield: $94.5 \%$ (31.8 mg, $189 \mu \mathrm{mol}), 85.8 \%$ (736 mg, $4.38 \mathrm{mmol})$;

colorless solid; 
$\operatorname{mp} 44-45^{\circ} \mathrm{C}$;

TLC $R_{\mathrm{f}} 0.65(n$-hexane/EtOAc $=20 / 1)$;

${ }^{1} \mathrm{H}$ NMR $\left(\mathrm{CDCl}_{3}, 400 \mathrm{MHz}\right): \delta 7.96(\mathrm{dd}, J=1.2,7.6 \mathrm{~Hz}, 2 \mathrm{H}), 7.58(\mathrm{~d}, J=8.0 \mathrm{~Hz}, 2 \mathrm{H}), 7.46(\mathrm{ddd}, J$ $=1.2,7.2,8.0 \mathrm{~Hz}, 2 \mathrm{H}), 7.35(\mathrm{dd}, J=7.2,7.6 \mathrm{~Hz}, 2 \mathrm{H})$;

${ }^{13} \mathrm{C}\left\{{ }^{1} \mathrm{H}\right\}$ NMR $\left(\mathrm{CDCl}_{3}, 100 \mathrm{MHz}\right): \delta 156.1$ (2C), 127.1 (2C), $124.2(2 \mathrm{C}), 122.6(2 \mathrm{C}), 120.6(2 \mathrm{C})$, $111.6(2 \mathrm{C})$.

The chemical shifts were consistent with those reported in the literature. ${ }^{\mathrm{S} 5}$

\section{2-Methyldibenzo $[b, d]$ furan $(2 \mathrm{~b}=\mathbf{2 h})$}<smiles>Cc1ccc2oc3ccccc3c2c1</smiles>

Purified by silica-gel column chromatography (hexane only);

Yield: $88.4 \%$ (from 1b, $16.1 \mathrm{mg}, 88.4 \mu \mathrm{mol}$ ), 81.8\% (from 1h, $14.9 \mathrm{mg}, 81.8 \mu \mathrm{mol}$ );

colorless solid:

$\operatorname{mp} 44-45^{\circ} \mathrm{C}$;

TLC $R_{\mathrm{f}} 0.65(n$-hexane/EtOAc $=20 / 1)$

${ }^{1} \mathrm{H} \mathrm{NMR}\left(\mathrm{CDCl}_{3}, 400 \mathrm{MHz}\right): \delta 7.91(\mathrm{ddd}, J=0.8,1.2,7.6 \mathrm{~Hz}, 1 \mathrm{H}), 7.75(\mathrm{dd}, J=0.8,0.8 \mathrm{~Hz}, 1 \mathrm{H})$, $7.54(\mathrm{ddd}, J=0.8,0.8,8.4 \mathrm{~Hz}, 1 \mathrm{H}), 7.47-7.41(\mathrm{~m}, 2 \mathrm{H}), 7.32(\mathrm{ddd}, J=0.8,7.6,7.6 \mathrm{~Hz}, 1 \mathrm{H}), 7.28-$ $7.24(\mathrm{~m}, 1 \mathrm{H}), 2.52(\mathrm{~s}, 3 \mathrm{H})$;

${ }^{13} \mathrm{C}\left\{{ }^{1} \mathrm{H}\right\} \mathrm{NMR}\left(\mathrm{CDCl}_{3}, 100 \mathrm{MHz}\right): \delta 156.4,154.5,132.2,128.2,126.9,124.22,124.15,122.5,120.6$, 120.5, 111.6, 111.1, 21.3;

IR $\left(\mathrm{cm}^{-1}\right)$ 2920, 1481, 1447, 1339, 1315, 1279, 1244, 1192, 1165, 1121, 1103, 1030, 872, 841, 802, 746, 723;

HRMS (EI) $m / z$ : [M] $]^{+}$calcd for $\mathrm{C}_{13} \mathrm{H}_{10} \mathrm{O}, 182.0732$; found, 182.0727.

The chemical shifts were consistent with those reported in the literature. ${ }^{\mathrm{S} 5}$

\section{Methyl dibenzo $[b, d]$ furan-2-carboxylate (2c)}<smiles>CC(=O)c1ccc2oc3ccccc3c2c1</smiles>

Purified by silica-gel column chromatography (hexane only);

Yield: $84.0 \%$ (19.0 mg, $84.0 \mu \mathrm{mol})$;

colorless solid;

$\operatorname{mp~} 81-82{ }^{\circ} \mathrm{C}$;

TLC $R_{\mathrm{f}} 0.60(n$-hexane/EtOAc $=10 / 1)$;

${ }^{1} \mathrm{H} \mathrm{NMR}\left(\mathrm{CDCl}_{3}, 400 \mathrm{MHz}\right): \delta 8.69(\mathrm{dd}, J=1.6,1.8 \mathrm{~Hz}, 1 \mathrm{H}), 8.19(\mathrm{dd}, J=1.6,8.8 \mathrm{~Hz}, 1 \mathrm{H}), 8.01$ (ddd, $J=0.8,0.8,7.6 \mathrm{~Hz}, 1 \mathrm{H}), 7.62-7.58(\mathrm{~m}, 2 \mathrm{H}), 7.51(\mathrm{ddd}, J=0.8,7.6,7.6 \mathrm{~Hz}, 1 \mathrm{H}), 7.40$ (ddd, $J$ $=0.8,7.6,7.6 \mathrm{~Hz}, 1 \mathrm{H}), 3.99(\mathrm{~s}, 3 \mathrm{H})$;

${ }^{13} \mathrm{C}\left\{{ }^{1} \mathrm{H}\right\} \mathrm{NMR}\left(\mathrm{CDCl}_{3}, 100 \mathrm{MHz}\right): \delta 167.0,158.9,156.8,128.9,127.9,125.0,124.4,123.7,123.3$, $122.9,120.9,111.9,111.5,52.2$;

IR $\left(\mathrm{cm}^{-1}\right) 2949,1713,1601,1589,1476,1449,1433,1352,1327,1304,1281,1238,1215,1194,1152$, 1107, 1090, 1022, 970, 907, 876, 841, 820, 770, 746, 721, 685;

HRMS (EI) $m / z$ : [M] $]^{+}$calcd for $\mathrm{C}_{14} \mathrm{H}_{10} \mathrm{O}_{3}, 226.0630$; found, 226.0629 .

The chemical shifts were consistent with those reported in the literature. ${ }^{\mathrm{S} 6}$ 


\section{2-(Trifluoromethyl)dibenzo $[b, d]$ furan (2d)}<smiles>FC(F)(F)c1ccc2oc3ccccc3c2c1</smiles>

Purified by silica-gel column chromatography (hexane only);

Yield: 95.3\% (22.5 mg, $95.3 \mu \mathrm{mol})$;

colorless solid;

mp $101-102{ }^{\circ} \mathrm{C}$;

TLC $R_{\mathrm{f}} 0.65(n$-hexane/EtOAc $=4 / 1)$;

${ }^{1} \mathrm{H}$ NMR $\left(\mathrm{CDCl}_{3}, 400 \mathrm{MHz}\right): \delta 8.24(\mathrm{dd}, J=0.8,0.8 \mathrm{~Hz}, 1 \mathrm{H}), 8.00(\mathrm{ddd}, J=0.8,1.2,7.6 \mathrm{~Hz}, 1 \mathrm{H})$, $7.73(\mathrm{dd}, J=0.8,8.8 \mathrm{~Hz}, 1 \mathrm{H}), 7.66(\mathrm{~d}, J=8.8 \mathrm{~Hz}, 1 \mathrm{H}), 7.62(\mathrm{ddd}, J=0.8,0.8,8.8 \mathrm{~Hz}, 1 \mathrm{H}), 7.53$ (ddd, $J=1.2,7.6,8.8 \mathrm{~Hz}, 1 \mathrm{H}), 7.41$, (ddd, $J=0.8,7.6,7.6 \mathrm{~Hz}, 1 \mathrm{H})$;

${ }^{13} \mathrm{C}\left\{{ }^{1} \mathrm{H}\right\} \mathrm{NMR}\left(\mathrm{CDCl}_{3}, 100 \mathrm{MHz}\right): \delta 157.6,156.8,128.2,125.3\left(\mathrm{q}, J_{\mathrm{C}-\mathrm{F}}=33.6 \mathrm{~Hz}\right), 124.543\left(\mathrm{q}, J_{\mathrm{C}-\mathrm{F}}\right.$ $=273.1 \mathrm{~Hz}), 124.538,124.2\left(\mathrm{q}, J_{\mathrm{C}-\mathrm{F}}=3.8 \mathrm{~Hz}\right), 123.4,123.3,120.9,118.3\left(\mathrm{q}, J_{\mathrm{C}-\mathrm{F}}=4.8 \mathrm{~Hz}\right), 112.03$, 111.98;

${ }^{19} \mathrm{~F}$ NMR $\left(\mathrm{CDCl}_{3}, 372.5 \mathrm{MHz}\right): \delta-61.1(\mathrm{~s})$;

IR $\left(\mathrm{cm}^{-1}\right) 721,1452,1352,1333,1319,1292,1267,1252,1207,1161,1152,1125,1096,1051,1022$, 1007, 976, 939, 897, 843, 827, 787, 772, 754, 737, 673, 650;

HRMS (EI) $m / z$ : [M] $]^{+}$calcd for $\mathrm{C}_{13} \mathrm{H}_{7} \mathrm{~F}_{3} \mathrm{O}, 236.0449$; found, 236.0454 .

The chemical shifts were consistent with those reported in the literature. ${ }^{\mathrm{S} 7}$

\section{2-Nitrodibenzo[b,d] furan (2e)}<smiles>O=[N+]([O-])c1ccc2oc3ccccc3c2c1</smiles>

Purified by silica-gel column chromatography (hexane only);

Yield: $64.1 \%$ (12.7 mg, $59.6 \mu \mathrm{mol})$;

colorless solid;

$\operatorname{mp} 152-153^{\circ} \mathrm{C}$;

TLC $R_{\mathrm{f}} 0.60(n$-hexane/EtOAc $=20 / 1)$;

${ }^{1} \mathrm{H} \mathrm{NMR}\left(\mathrm{CDCl}_{3}, 400 \mathrm{MHz}\right): \delta 8.88(\mathrm{~d}, J=2.4 \mathrm{~Hz}, 1 \mathrm{H}), 8.40(\mathrm{dd}, J=2.4,9.2 \mathrm{~Hz}, 1 \mathrm{H}), 8.03(\mathrm{ddd}, J$ $=0.8,0.8,8.0 \mathrm{~Hz}, 1 \mathrm{H}), 7.69-7.62(\mathrm{~m}, 2 \mathrm{H}), 7.58(\mathrm{ddd}, J=0.8,8.0,8.0 \mathrm{~Hz}, 1 \mathrm{H}), 7.46$ (ddd, $J=1.2$, $8.0,8.0 \mathrm{~Hz}, 1 \mathrm{H})$;

${ }^{13} \mathrm{C}\left\{{ }^{1} \mathrm{H}\right\} \mathrm{NMR}\left(\mathrm{CDCl}_{3}, 100 \mathrm{MHz}\right): \delta 159.2,157.5,143.8,128.9,125.0,123.9,123.1,123.0,121.3$, 117.1, 112.2, 112.0;

IR $\left(\mathrm{cm}^{-1}\right) 1601,1585,1524,1466,1443,1362,1339,1317,1296,1250,1194,1184,1018,893,841$, 820, 766, 745, 718, 689;

HRMS (EI) $m / z$ : [M] $]^{+}$calcd for $\mathrm{C}_{12} \mathrm{H}_{7} \mathrm{NO}_{3}, 213.0426$; found, 213.0424 .

The chemical shifts were consistent with those reported in the literature. ${ }^{\mathrm{S} 8}$

\section{3-(Trifluoromethyl)dibenzo[b,d]furan (2f)}<smiles>FC(F)(F)c1ccc2c(c1)oc1ccccc12</smiles>

Purified by silica-gel column chromatography (hexane only); 
Yield: $64.8 \%$ (15.3 mg, $64.8 \mu \mathrm{mol})$;

colorless solid;

mp $114-115^{\circ} \mathrm{C}$;

TLC $R_{\mathrm{f}} 0.60(n$-hexane/EtOAc $=10 / 1)$;

${ }^{1} \mathrm{H}$ NMR $\left(\mathrm{CDCl}_{3}, 400 \mathrm{MHz}\right): \delta 8.05(\mathrm{~d}, J=8.0 \mathrm{~Hz}, 1 \mathrm{H}), 8.00(\mathrm{dd}, J=0.8,8.0 \mathrm{~Hz}, 1 \mathrm{H}), 7.84(\mathrm{~d}, J=$ $0.8 \mathrm{~Hz}, 1 \mathrm{H}), 7.64-7.59$ (m, 2H), $7.54(\mathrm{ddd}, J=1.2,7.2,8.4 \mathrm{~Hz}, 1 \mathrm{H}), 7.40$ (ddd, $J=1.2,7.2,8.4 \mathrm{~Hz}$, $1 \mathrm{H})$;

${ }^{13} \mathrm{C}\left\{{ }^{1} \mathrm{H}\right\} \mathrm{NMR}\left(\mathrm{CDCl}_{3}, 100 \mathrm{MHz}\right): \delta 157.1,155.4,128.7\left(\mathrm{q}, J_{\mathrm{C}-\mathrm{F}}=45.0 \mathrm{~Hz}\right), 128.5,127.4,124.2(\mathrm{q}$, $\left.J_{\mathrm{C}-\mathrm{F}}=274.0 \mathrm{~Hz}\right), 123.3,123.1,121.3,121.0,119.7\left(\mathrm{q}, J_{\mathrm{C}-\mathrm{F}}=3.8 \mathrm{~Hz}\right), 112.0,109.2\left(\mathrm{q}, J_{\mathrm{C}-\mathrm{F}}=3.8 \mathrm{~Hz}\right)$; ${ }^{19} \mathrm{~F} \mathrm{NMR}\left(\mathrm{CDCl}_{3}, 372.5 \mathrm{MHz}\right): \delta-61.6(\mathrm{~s})$;

IR $\left(\mathrm{cm}^{-1}\right) 1427,1335,1229,1163,1150,1126,1101,1055,907,883,831,772,750,727$;

HRMS (EI) $m / z$ : [M] $]^{+}$calcd for $\mathrm{C}_{13} \mathrm{H}_{7} \mathrm{~F}_{3} \mathrm{O}, 236.0449$; found, 236.0457.

The chemical shifts were consistent with those reported in the literature. ${ }^{\mathrm{S} 8}$

\section{4-Phenyldibenzo $[b, d]$ furan $(2 \mathrm{~g})$}<smiles>c1ccc2c(c1)oc1ccccc12</smiles>

Purified by silica-gel column chromatography (hexane only);

Yield: $81.5 \%$ (19.9 mg, $81.5 \mu \mathrm{mol})$;

colorless oil;

TLC $R_{\mathrm{f}} 0.60(n$-hexane/EtOAc $=10 / 1)$;

${ }^{1} \mathrm{H} \mathrm{NMR}\left(\mathrm{CDCl}_{3}, 400 \mathrm{MHz}\right): \delta 8.00(\mathrm{dd}, J=0.8,7.6 \mathrm{~Hz}, 1 \mathrm{H}), 7.96-7.90(\mathrm{~m}, 3 \mathrm{H}), 7.62-7.59(\mathrm{~m}, 2 \mathrm{H})$, $7.55(\mathrm{dd}, J=7.6,7.6 \mathrm{~Hz}, 2 \mathrm{H}), 7.49-7.41(\mathrm{~m}, 3 \mathrm{H}), 7.37(\mathrm{ddd}, J=0.8,7.6,7.6 \mathrm{~Hz}, 1 \mathrm{H})$;

${ }^{13} \mathrm{C}\left\{{ }^{1} \mathrm{H}\right\} \mathrm{NMR}\left(\mathrm{CDCl}_{3}, 100 \mathrm{MHz}\right): \delta 156.2,153.4,136.4,128.8(2 \mathrm{C}), 128.6(2 \mathrm{C}), 127.8,127.2,126.9$, $125.9,124.9,124.2,123.2,122.7,120.7,119.7,111.8$

IR $\left(\mathrm{cm}^{-1}\right) 3057,3030,2926,1744,1599,1585,1504,1485,1447,1406,1260,1188,1175,1061,1015$, 872, 843, 748, 731, 694;

HRMS (EI) $m / z$ : [M] ${ }^{+}$calcd for $\mathrm{C}_{18} \mathrm{H}_{12} \mathrm{O}, 244.0888$; found, 244.0892.

The chemical shifts were consistent with those reported in the literature. ${ }^{\mathrm{S} 9}$

\section{2-Methoxydibenzo $[b, d]$ furan (2i)}<smiles>COc1ccc2oc3ccccc3c2c1</smiles>

Purified by silica-gel column chromatography (hexane only);

Yield: 91.3\% (18.1 mg, $91.3 \mu \mathrm{mol})$;

colorless solid;

mp $45-46{ }^{\circ} \mathrm{C}$;

TLC $R_{\mathrm{f}} 0.70(n$-hexane/EtOAc $=5 / 1)$;

${ }^{1} \mathrm{H}$ NMR $\left(\mathrm{CDCl}_{3}, 400 \mathrm{MHz}\right): \delta 7.92(\mathrm{ddd}, J=0.8,1.2,8.0 \mathrm{~Hz}, 1 \mathrm{H}), 7.48-7.44(\mathrm{~m}, 2 \mathrm{H}), 7.54$ (ddd, $J$ $=0.8,0.8,8.0 \mathrm{~Hz}, 1 \mathrm{H}), 7.42(\mathrm{~d}, J=2.8 \mathrm{~Hz}, 1 \mathrm{H}), 7.32(\mathrm{ddd}, J=0.8,8.0,8.0 \mathrm{~Hz}, 1 \mathrm{H}), 7.05(\mathrm{dd}, J=$ 2.8, $8.8 \mathrm{~Hz}, 1 \mathrm{H}), 3.92(\mathrm{~s}, 3 \mathrm{H})$; 
${ }^{13} \mathrm{C}\left\{{ }^{1} \mathrm{H}\right\} \mathrm{NMR}\left(\mathrm{CDCl}_{3}, 100 \mathrm{MHz}\right): \delta 156.9,155.8,150.9,127.1,124.7,124.5,122.4,120.5,115.1$, 112.1, 111.7, 103.7, 56.0;

IR $\left(\mathrm{cm}^{-1}\right) 3057,2997,2936,2853,2832,1634,1601,1589,1481,1452,1437,1317,1296,1273,1250$, $1227,1186,1165,1150,1121,1103,1032,1016,860,841,808,745,723$;

HRMS (EI) $m / z$ : [M] $]^{+}$calcd for $\mathrm{C}_{13} \mathrm{H}_{10} \mathrm{O}_{2}, 198.0681$; found, 198.0680.

The chemical shifts were consistent with those reported in the literature. ${ }^{\mathrm{S} 5}$

\section{2-Fluorodibenzo $[b, d]$ furan $(2 \mathrm{j})$}<smiles>Fc1ccc2oc3ccccc3c2c1</smiles>

Purified by silica-gel column chromatography (hexane only);

Yield: 94.5\% (17.6 mg, $94.5 \mu \mathrm{mol})$;

colorless solid;

$\operatorname{mp} 88-90{ }^{\circ} \mathrm{C}$;

TLC $R_{\mathrm{f}} 0.70(n$-hexane/EtOAc $=20 / 1)$;

${ }^{1} \mathrm{H}$ NMR $\left(\mathrm{CDCl}_{3}, 400 \mathrm{MHz}\right): \delta 7.92(\mathrm{ddd}, J=0.8,1.2,7.6 \mathrm{~Hz}, 1 \mathrm{H}), 7.61(\mathrm{dd}, J=2.8,8.4 \mathrm{~Hz}, 1 \mathrm{H})$, $7.57(\mathrm{~d}, J=8.4 \mathrm{~Hz}, 1 \mathrm{H}), 7.52-7.46(\mathrm{~m}, 2 \mathrm{H}), 7.35(\mathrm{ddd}, J=1.2,7.6,8.8 \mathrm{~Hz}, 1 \mathrm{H}), 7.17(\mathrm{ddd}, J=2.8$, $8.8,8.8 \mathrm{~Hz}, 1 \mathrm{H})$;

${ }^{13} \mathrm{C}\left\{{ }^{1} \mathrm{H}\right\} \mathrm{NMR}\left(\mathrm{CDCl}_{3}, 100 \mathrm{MHz}\right): \delta 159.0(\mathrm{~d}, J=240.4 \mathrm{~Hz}), 157.2,152.2,127.8,125.1\left(\mathrm{~d}, J_{\mathrm{C}-\mathrm{F}}=\right.$ $10.5 \mathrm{~Hz}), 124.0\left(\mathrm{~d}, J_{\mathrm{C}-\mathrm{F}}=2.8 \mathrm{~Hz}\right), 122.8,120.8,114.4\left(\mathrm{~d}, J_{\mathrm{C}-\mathrm{F}}=25.9 \mathrm{~Hz}\right), 112.3,\left(\mathrm{~d}, J_{\mathrm{C}-\mathrm{F}}=9.5 \mathrm{~Hz}\right)$, $111.9,106.7\left(\mathrm{~d}, J_{\mathrm{C}-\mathrm{F}}=24.9 \mathrm{~Hz}\right)$;

${ }^{19} \mathrm{~F} \mathrm{NMR}\left(\mathrm{CDCl}_{3}, 372.5 \mathrm{MHz}\right): \delta-120.8(\mathrm{dd}, J=8.6,14.5 \mathrm{~Hz})$;

IR $\left(\mathrm{cm}^{-1}\right)$ 1477, 1447, 1209, 1159, 864, 808, 746;

HRMS (EI) $m / z$ : [M] $]^{+}$calcd for $\mathrm{C}_{12} \mathrm{H}_{7} \mathrm{FO}, 186.0481$; found, 186.0486 .

The chemical shifts were consistent with those reported in the literature. ${ }^{\mathrm{S} 10}$

\section{2-Fluoro-8-methyldibenzo[b,d]furan (2k)}<smiles>Cc1ccc2oc3ccc(F)cc3c2c1</smiles>

Purified by silica-gel column chromatography (hexane only);

Yield: 71.9\% (14.4 mg, $71.9 \mu \mathrm{mol})$;

colorless solid;

$\operatorname{mp} 71-72^{\circ} \mathrm{C}$;

TLC $R_{\mathrm{f}} 0.65(n$-hexane/EtOAc $=20 / 1)$;

${ }^{1} \mathrm{H} \mathrm{NMR}\left(\mathrm{CDCl}_{3}, 400 \mathrm{MHz}\right): \delta 7.70(\mathrm{dd}, J=0.8,0.8 \mathrm{~Hz}, 1 \mathrm{H}), 7.56(\mathrm{dd}, J=2.8,8.0 \mathrm{~Hz}, 1 \mathrm{H}), 7.49-$ $7.42(\mathrm{~m}, 2 \mathrm{H}), 7.30-7.27(\mathrm{~m}, 1 \mathrm{H}), 7.14$ (ddd, $J=2.8,8.8,8.8 \mathrm{~Hz}, 1 \mathrm{H}), 2.51(\mathrm{~s}, 3 \mathrm{H})$;

${ }^{13} \mathrm{C}\left\{{ }^{1} \mathrm{H}\right\} \mathrm{NMR}\left(\mathrm{CDCl}_{3}, 100 \mathrm{MHz}\right): \delta 158.9\left(\mathrm{~d}, J_{\mathrm{C}-\mathrm{F}}=240.4 \mathrm{~Hz}\right), 155.5,152.5,132.3,128.9,125.1(\mathrm{~d}$, $\left.J_{\mathrm{C}-\mathrm{F}}=9.6 \mathrm{~Hz}\right), 123.9\left(\mathrm{~d}, J_{\mathrm{C}-\mathrm{F}}=3.9 \mathrm{~Hz}\right), 120.8,114.2\left(\mathrm{~d}, J_{\mathrm{C}-\mathrm{F}}=25.9 \mathrm{~Hz}\right), 112.2\left(\mathrm{~d}, J_{\mathrm{C}-\mathrm{F}}=8.6 \mathrm{~Hz}\right)$, $111.4,106.5\left(\mathrm{~d}, J_{\mathrm{C}-\mathrm{F}}=24.8 \mathrm{~Hz}\right), 21.3$;

${ }^{19} \mathrm{~F} \mathrm{NMR}\left(\mathrm{CDCl}_{3}, 372.5 \mathrm{MHz}\right): \delta-121.1(\mathrm{dd}, J=8.6,8.6 \mathrm{~Hz})$;

IR $\left(\mathrm{cm}^{-1}\right) 2922,1595,1485,1456,1307,1265,1246,1223,1196,1177,1136,1121,1101,930,907$, 
$864,853,808,781,750,735,723$;

HRMS (EI) $m / z$ : [M] ${ }^{+}$calcd for $\mathrm{C}_{13} \mathrm{H}_{9} \mathrm{FO}, 200.0637$; found, 200.0633 .

\section{1-Methoxy-8-methoxycarbonyl-3-methyldibenzo $[b, d]$ furan (2I)}<smiles>COC(=O)c1ccc2oc3cc(C)cc(OC)c3c2c1</smiles>

Purified by silica-gel column chromatography (hexane only);

Yield: $78.9 \%$ (9.3 mg, $34.4 \mu \mathrm{mol})$;

colorless solid;

mp $132-134{ }^{\circ} \mathrm{C}$;

TLC $R_{\mathrm{f}} 0.45(n$-hexane/EtOAc $=20 / 1)$;

${ }^{1} \mathrm{H} \mathrm{NMR}\left(\mathrm{CDCl}_{3}, 400 \mathrm{MHz}\right): \delta 8.74(\mathrm{~d}, J=1.6 \mathrm{~Hz}, 1 \mathrm{H}), 8.11(\mathrm{dd}, J=1.6,8.8 \mathrm{~Hz}, 1 \mathrm{H}), 7.52(\mathrm{~d}, J=$ $8.8 \mathrm{~Hz}, 1 \mathrm{H}), 7.01(\mathrm{~s}, 1 \mathrm{H}), 6.65(\mathrm{~s}, 1 \mathrm{H}), 4.06(\mathrm{~s}, 3 \mathrm{H}), 3.97(\mathrm{~s}, 3 \mathrm{H}), 2.52(\mathrm{~s}, 3 \mathrm{H})$;

${ }^{13} \mathrm{C}\left\{{ }^{1} \mathrm{H}\right\} \mathrm{NMR}\left(\mathrm{CDCl}_{3}, 100 \mathrm{MHz}\right): \delta 167.3,158.2,158.1,155.5,139.6,127.6,125.0,124.4,123.9$, 110.7, 110.6, 105.7, 104.7, 55.6, 52.1, 22.5;

IR $\left(\mathrm{cm}^{-1}\right) 2911,1710,1610,1599,1436,1339,1309,1286,1249,1227,1191,1106,1083,1012,966$, $813,768,720,643,589,571$;

HRMS $\left(\mathrm{ESI}^{+}\right) \mathrm{m} / z:[\mathrm{M}+\mathrm{Na}]^{+}$calcd for $\mathrm{C}_{16} \mathrm{H}_{14} \mathrm{NaO}_{4}{ }^{+}, 293.0784$; found, 293.0779 .

\section{1,6-Dimethoxy-3,8-dimethyldibenzo[b,d]furan (2m)}<smiles>COc1cc(C)cc2c1oc1cc(C)cc(OC)c12</smiles>

Purified by silica-gel column chromatography (hexane only);

Yield: 53.0\% (13.6 mg, $53.0 \mu \mathrm{mol})$;

colorless solid;

mp $152-154{ }^{\circ} \mathrm{C}$;

TLC $R_{\mathrm{f}} 0.55(n$-hexane/EtOAc $=20 / 1)$;

${ }^{1} \mathrm{H} \mathrm{NMR}\left(\mathrm{CDCl}_{3}, 400 \mathrm{MHz}\right): \delta 7.47(\mathrm{~d}, J=1.2 \mathrm{~Hz}, 1 \mathrm{H}), 7.00(\mathrm{~d}, J=0.8 \mathrm{~Hz}, 1 \mathrm{H}), 6.74(\mathrm{~d}, J=1.2 \mathrm{~Hz}$, $1 \mathrm{H}), 6.58(\mathrm{br}, 1 \mathrm{H}), 4.03(\mathrm{~s}, 3 \mathrm{H}), 4.02(\mathrm{~s}, 3 \mathrm{H}), 2.50(\mathrm{~s}, 3 \mathrm{H}), 2.49(\mathrm{~s}, 3 \mathrm{H})$;

${ }^{13} \mathrm{C}\left\{{ }^{1} \mathrm{H}\right\} \mathrm{NMR}\left(\mathrm{CDCl}_{3}, 100 \mathrm{MHz}\right): \delta 157.8,155.2,144.4,142.5,138.5,133.2,125.0,114.6,111.3$, 109.6, 105.1, 104.9, 56.1, 55.5, 22.4, 21.8;

IR $\left(\mathrm{cm}^{-1}\right)$ 2975, 2917, 1637, 1595, 1454, 1405, 1349, 1306, 1231, 1207, 1091, 1044, 878, 669, 598; HRMS $\left(\mathrm{ESI}^{+}\right) \mathrm{m} / \mathrm{z}:[\mathrm{M}+\mathrm{Na}]^{+}$calcd for $\mathrm{C}_{16} \mathrm{H}_{16} \mathrm{NaO}_{3}{ }^{+}, 279.0992$; found, 279.0986. 


\section{4-Benzyloxy-5,7-dimethoxy-9-methylnaphtho[1,2-b]benzofuran (2n)}<smiles></smiles>

Purified by silica-gel column chromatography (hexane $/$ EtOAc $=5 / 1$ );

Yield: $43.6 \%$ (17.3 mg, $43.6 \mu \mathrm{mol})$;

colorless solid;

mp $137-139{ }^{\circ} \mathrm{C}$;

TLC $R_{\mathrm{f}} 0.55(n$-hexane/EtOAc $=5 / 1)$;

${ }^{1} \mathrm{H}$ NMR $\left(\mathrm{CDCl}_{3}, 400 \mathrm{MHz}\right): \delta 8.00(\mathrm{dd}, J=0.8,8.4 \mathrm{~Hz}, 1 \mathrm{H}), 7.64(\mathrm{dd}, J=0.8,7.6 \mathrm{~Hz}, 2 \mathrm{H}), 7.54(\mathrm{~s}$, $1 \mathrm{H}), 7.51(\mathrm{dd}, J=8.4,8.4 \mathrm{~Hz}, 1 \mathrm{H}), 7.43(\mathrm{dd}, J=7.6,7.6 \mathrm{~Hz}, 2 \mathrm{H}), 7.34(\mathrm{t}, J=7.6 \mathrm{~Hz}, 1 \mathrm{H}), 7.10(\mathrm{~s}$, $1 \mathrm{H}), 7.02(\mathrm{dd}, J=0.8,8.4 \mathrm{~Hz}, 1 \mathrm{H}), 6.63(\mathrm{~s}, 1 \mathrm{H}), 5.26(\mathrm{~s}, 2 \mathrm{H}), 4.07(\mathrm{~s}, 3 \mathrm{H}), 4.05(\mathrm{~s}, 3 \mathrm{H}), 2.54(\mathrm{~s}, 3 \mathrm{H})$; ${ }^{13} \mathrm{C}\left\{{ }^{1} \mathrm{H}\right\}$ NMR $\left(\mathrm{CDCl}_{3}, 100 \mathrm{MHz}\right): \delta 157.7,156.6,155.0,153.7,145.8,137.8,137.6,128.4(2 \mathrm{C})$, 127.6, 127.09 (2C), 127.05, 123.9, 118.7, 116.5, 114.0, 112.3, 108.8, 105.0 (2C), 100.8, 71.6, 57.2, 55.6, 22.5;

IR $\left(\mathrm{cm}^{-1}\right)$ 2926, 2855, 1584, 1524, 1443, 1406, 1342, 1298, 1285, 1260, 1231, 1206, 1128, 1094, 1057, 1042, 1028, 986, 816, 750, 725, 694;

HRMS (EI) $m / z$ : [M] $]^{+}$calcd for $\mathrm{C}_{26} \mathrm{H}_{22} \mathrm{O}_{4}, 398.1518$; found, 398.1509.

\section{Benzofuro[3,2-b]pyridine (2o)}<smiles>c1cnc2c(c1)oc1cccnc12</smiles>

Purified by silica-gel column chromatography (hexane/EtOAc $=5 / 1$ );

Yield: $51.8 \%$ (26.3 mg, $15.5 \mu \mathrm{mol})$;

colorless solid;

mp $60-61^{\circ} \mathrm{C}$;

TLC $R_{\mathrm{f}} 0.20(n$-hexane/EtOAc $=10 / 1)$;

${ }^{1} \mathrm{H}$ NMR $\left(\mathrm{CDCl}_{3}, 400 \mathrm{MHz}\right): \delta 8.64(\mathrm{dd}, J=1.2,5.2 \mathrm{~Hz}, 1 \mathrm{H}), 8.25(\mathrm{~d}, J=8.0 \mathrm{~Hz}, 1 \mathrm{H}), 7.84(\mathrm{dd}, J=$ $1.2,8.4 \mathrm{~Hz}, 1 \mathrm{H}), 7.63-7.54(\mathrm{~m}, 2 \mathrm{H}), 7.44$ (ddd, $J=1.2,8.0,8.0 \mathrm{~Hz}, 1 \mathrm{H}), 7.38$ (dd, $J=5.2,8.4 \mathrm{~Hz}$, $1 \mathrm{H})$;

${ }^{13} \mathrm{C}\left\{{ }^{1} \mathrm{H}\right\} \mathrm{NMR}\left(\mathrm{CDCl}_{3}, 100 \mathrm{MHz}\right): \delta 157.4,149.7,145.2,144.2,129.2,123.6,123.3,121.24,121.16$, 118.6, 112.1;

IR $\left(\mathrm{cm}^{-1}\right) 3084,3019,1628,1591,1470,1449,1400,1373,1346,1317,1294,1263,1240,1194$, 1146, 1103, 1043, 1005, 964, 943, 924, 868, 841, 783, 752, 729, 664, 654;

HRMS (EI) $m / z$ : [M] $]^{+}$calcd for $\mathrm{C}_{11} \mathrm{H}_{7} \mathrm{NO}, 169.0528$; found, 169.0526.

The chemical shifts were consistent with those reported in the literature. ${ }^{\mathrm{S} 11}$ 


\section{2-Bromo-6-hydroxy-6H-dibenzo $[c, e][1,2]$ oxaborin $(1 \mathrm{q})$}<smiles>OB1Oc2ccc(Br)cc2-c2ccccc21</smiles>

Colorless solid;

$\mathrm{mp} 246-248^{\circ} \mathrm{C}$;

TLC $R_{\mathrm{f}} 0-0.16$ (broad spot, $n$-hexane/EtOAc $\left.=5 / 1\right)$;

${ }^{1} \mathrm{H}$ NMR (acetone- $\left.d_{6}, 500 \mathrm{MHz}\right): \delta 8.42(\mathrm{~d}, J=2.5 \mathrm{~Hz}, 1 \mathrm{H}), 8.41(\mathrm{~s}, 1 \mathrm{H}), 8.36(\mathrm{~d}, J=8.5 \mathrm{~Hz}, 1 \mathrm{H})$, $8.17(\mathrm{~d}, J=7.0 \mathrm{~Hz}, 1 \mathrm{H}), 7.79(\mathrm{dd}, J=8.5,8.5 \mathrm{~Hz}, 1 \mathrm{H}), 7.59-7.53(\mathrm{~m}, 2 \mathrm{H}), 7.23(\mathrm{~d}, J=8.5 \mathrm{~Hz}, 1 \mathrm{H})$; ${ }^{13} \mathrm{C}\left\{{ }^{1} \mathrm{H}\right\}$ NMR (acetone- $\left.d_{6}, 126 \mathrm{MHz}\right): \delta 152.4,140.6,135.1,134.3,133.2,129.6,128.1,126.7,123.7$, $123.1,116.4$ (the signal for the carbon attached to the boron atom was not observed);

${ }^{11} \mathrm{~B}$ NMR (acetone- $\left.d_{6}, 128 \mathrm{MHz}\right): \delta 27.9$;

IR $\left(\mathrm{KBr}, \mathrm{cm}^{-1}\right) 3298,3058,1608,1486,1438,1371,1325,1310,1294,1277,801$;

Anal. Calcd for $\mathrm{C}_{12} \mathrm{H}_{8} \mathrm{BBrO}_{2}$ : C, 52.43; H, 2.93\%. Found: C, 52.43; H, 3.22\%.

\section{6-Hydroxy-2-iodo-6H-dibenzo $[c, e][1,2]$ oxaborin (1r)}<smiles>OB1Oc2ccc(I)cc2-c2ccccc21</smiles>

Colorless solid;

mp 249-251 ${ }^{\circ} \mathrm{C}$;

TLC $R_{\mathrm{f}} 0-0.19$ (broad spot, $n$-hexane/EtOAc $=5 / 1$ );

${ }^{1} \mathrm{H}$ NMR (acetone- $\left.d_{6}, 500 \mathrm{MHz}\right): \delta 8.59(\mathrm{~d}, J=2.0 \mathrm{~Hz}, 1 \mathrm{H}), 8.41(\mathrm{~s}, 1 \mathrm{H}), 8.34(\mathrm{~d}, J=8.0 \mathrm{~Hz}, 1 \mathrm{H})$, $8.16(\mathrm{~d}, J=8.5 \mathrm{~Hz}, 1 \mathrm{H}), 7.78(\mathrm{dd}, J=8.0,8.0 \mathrm{~Hz}, 1 \mathrm{H}), 7.72(\mathrm{dd}, J=2.0,8.5 \mathrm{~Hz}, 1 \mathrm{H}), 7.56(\mathrm{dd}, J=$ $8.0,8.0 \mathrm{~Hz}, 1 \mathrm{H}), 7.09(\mathrm{~d}, J=8.0 \mathrm{~Hz}, 1 \mathrm{H})$;

${ }^{13} \mathrm{C}\left\{{ }^{1} \mathrm{H}\right\}$ NMR (acetone- $d_{6}, 126 \mathrm{MHz}$ ): $\delta 153.0,140.5,139.2,135.1,134.2,134.1,129.5,127.2,123.6$, $123.5,86.6$ (the signal for the carbon attached to the boron atom was not observed);

${ }^{11} \mathrm{~B}$ NMR (acetone- $\left.d_{6}, 128 \mathrm{MHz}\right): \delta 30.1$;

IR $\left(\mathrm{KBr}, \mathrm{cm}^{-1}\right) 3396,3316,1606,1485,1439,1364,1328,1312,1298,1278,826,815$;

HRMS $\left(\mathrm{ESI}^{-}\right) \mathrm{m} / z$ : $[\mathrm{M}-\mathrm{H}]^{-}$calcd for $\mathrm{C}_{12} \mathrm{H}_{7} \mathrm{BIO}_{2}, 320.9584$; found, 320.9582 .

\section{6-Hydroxy-2-(thiophen-2-yl)-6H-dibenzo[c,e][1,2]oxaborin (1s)}<smiles>B1Oc2ccc(-c3cccs3)cc2-c2ccccc21</smiles>

Pale gray solid;

mp 224-225 ${ }^{\circ} \mathrm{C}$;

TLC $R_{\mathrm{f}} 0-0.19$ (broad spot, $n$-hexane $/$ EtOAc $=5 / 1$ );

${ }^{1} \mathrm{H}$ NMR (acetone- $\left.d_{6}, 500 \mathrm{MHz}\right): \delta 8.55(\mathrm{~d}, J=2.5 \mathrm{~Hz}, 1 \mathrm{H}), 8.46(\mathrm{~d}, J=8.5 \mathrm{~Hz}, 1 \mathrm{H}), 8.36(\mathrm{~s}, 1 \mathrm{H})$, $8.18(\mathrm{~d}, J=8.5 \mathrm{~Hz}, 1 \mathrm{H}), 7.80(\mathrm{dd}, J=8.5,8.5 \mathrm{~Hz}, 1 \mathrm{H}),, 7.71(\mathrm{dd}, J=2.5,8.5 \mathrm{~Hz}, 1 \mathrm{H}), 7.58-7.53(\mathrm{~m}$, $2 \mathrm{H}), 7.48(\mathrm{dd}, J=1.0,5.0 \mathrm{~Hz}, 1 \mathrm{H}), 7.32(\mathrm{~d}, J=8.5 \mathrm{~Hz}, 1 \mathrm{H}), 7.17(\mathrm{dd}, J=3.5,5.0 \mathrm{~Hz}, 1 \mathrm{H})$;

${ }^{13} \mathrm{C}\left\{{ }^{1} \mathrm{H}\right\}$ NMR (acetone- $\left.d_{6}, 126 \mathrm{MHz}\right): \delta 152.8,145.4,141.5,135.1,134.1,130.7,129.8,129.1,128.3$, $126.3,124.90,124.86,123.6,122.4,121.8$ (the signal for the carbon attached to the boron atom was 
not observed);

${ }^{11} \mathrm{~B}$ NMR (acetone- $\left.d_{6}, 128 \mathrm{MHz}\right): \delta 28.0$;

IR $\left(\mathrm{KBr}, \mathrm{cm}^{-1}\right) 3456,1603,1492,1444,1393,1312,1242,1022,846,819$;

HRMS (ESI $\left.{ }^{-}\right) \mathrm{m} / z$ : $[\mathrm{M}-\mathrm{H}]^{-}$calcd for $\mathrm{C}_{16} \mathrm{H}_{10} \mathrm{BO}_{2} \mathrm{~S}, 277.0495$; found, 277.0498.

\section{6-Hydroxy-2-[(trimethylsilyl)ethynyl]-6H-dibenzo[c,e][1,2]oxaborin (1t)}<smiles>C[SiH2]C#Cc1ccc2c(c1)-c1ccccc1B(O)O2</smiles>

Pale gray solid;

mp 232-234 ${ }^{\circ} \mathrm{C}$;

TLC $R_{\mathrm{f}} 0-0.22$ (broad spot, $n$-hexane/EtOAc $=5 / 1$ );

${ }^{1} \mathrm{H}$ NMR (acetone- $\left.d_{6}, 500 \mathrm{MHz}\right): \delta 8.43(\mathrm{~s}, 1 \mathrm{H}), 8.38-8.35(\mathrm{~m}, 2 \mathrm{H}), 8.16(\mathrm{~d}, J=8.0 \mathrm{~Hz}, 1 \mathrm{H}), 7.79$ $(\mathrm{dd}, J=8.5,8.5 \mathrm{~Hz}, 1 \mathrm{H}), 7.56(\mathrm{dd}, J=8.5,8.5 \mathrm{~Hz}, 1 \mathrm{H}), 7.50(\mathrm{dd}, J=2.0,8.0 \mathrm{~Hz}, 1 \mathrm{H}), 7.26(\mathrm{~d}, J=$ $8.5 \mathrm{~Hz}, 1 \mathrm{H}), 0.28(\mathrm{~s}, 9 \mathrm{H})$;

${ }^{13} \mathrm{C}\left\{{ }^{1} \mathrm{H}\right\}$ NMR (acetone- $\left.d_{6}, 126 \mathrm{MHz}\right): \delta 153.5,140.9,135.1,134.2,133.9,129.3,129.1,124.8,123.6$, $121.5,119.0,106.7,94.1,0.8(3 \mathrm{C})$ (the signal for the carbon attached to the boron atom was not observed);

${ }^{11} \mathrm{~B}$ NMR (acetone- $\left.d_{6}, 128 \mathrm{MHz}\right): \delta 28.1$;

IR $\left(\mathrm{KBr}, \mathrm{cm}^{-1}\right) 3443,2958,2156,1605,1491,1446,1376,1311,1249,901,841$;

HRMS (ESI $\left.{ }^{-}\right) m / z$ : $[\mathrm{M}-\mathrm{H}]^{-}$calcd for $\mathrm{C}_{17} \mathrm{H}_{16} \mathrm{BO}_{2} \mathrm{Si}, 291.1013$; found, 291.1014 .

\section{2-Bromodibenzo $[b, d]$ furan $(2 q)$}<smiles>Brc1ccc2oc3ccccc3c2c1</smiles>

Purified by silica-gel column chromatography (hexane only);

Yield: $95.5 \%$ (23.6 mg, $95.5 \mu \mathrm{mol})$;

colorless solid;

mp $108-109^{\circ} \mathrm{C}$;

TLC $R_{\mathrm{f}} 0.70(n$-hexane/EtOAc $=20 / 1)$;

${ }^{1} \mathrm{H}$ NMR $\left(\mathrm{CDCl}_{3}, 400 \mathrm{MHz}\right): \delta 8.08(\mathrm{~d}, J=2.0 \mathrm{~Hz}, 1 \mathrm{H}), 7.91(\mathrm{~d}, J=7.6 \mathrm{~Hz}, 1 \mathrm{H}), 7.59-7.53(\mathrm{~m}, 2 \mathrm{H})$, $7.52-7.43(\mathrm{~m}, 2 \mathrm{H}), 7.36$ (ddd, $J=0.8,7.6,7.6 \mathrm{~Hz}, 1 \mathrm{H})$;

${ }^{13} \mathrm{C}\left\{{ }^{1} \mathrm{H}\right\} \mathrm{NMR}\left(\mathrm{CDCl}_{3}, 100 \mathrm{MHz}\right): \delta 156.6,154.9,129.9,127.9,126.2,123.5,123.2,123.0,120.8$, $115.5,113.2,111.8$

IR $\left(\mathrm{cm}^{-1}\right) 1462,1437,1418,1265,1244,1200,1148,1121,1103,1051,935,880,856,841,810,766$, 752, 723, 681;

HRMS (EI) $m / z$ : [M] $]^{+}$calcd for $\mathrm{C}_{12} \mathrm{H}_{7}{ }^{79} \mathrm{BrO}, 245.9680$; found, 245.9680 .

The chemical shifts were consistent with those reported in the literature. ${ }^{\mathrm{S} 12}$ 


\section{2-Iododibenzo $[b, d]$ furan (2r)}<smiles>Ic1ccc2oc3ccccc3c2c1</smiles>

Purified by silica-gel column chromatography (hexane only);

Yield: $92.8 \%(27.3 \mathrm{mg}, 92.8 \mu \mathrm{mol})$;

colorless solid;

mp $104-105^{\circ} \mathrm{C}$;

TLC $R_{\mathrm{f}} 0.75$ ( $n$-hexane/EtOAc $\left.=20 / 1\right)$;

${ }^{1} \mathrm{H}$ NMR $\left(\mathrm{CDCl}_{3}, 400 \mathrm{MHz}\right): \delta 8.28(\mathrm{dd}, J=0.8,1.6 \mathrm{~Hz}, 1 \mathrm{H}), 7.91(\mathrm{ddd}, J=0.8,1.2,7.6 \mathrm{~Hz}, 1 \mathrm{H})$, $7.73(\mathrm{dd}, J=1.6,8.8 \mathrm{~Hz}, 1 \mathrm{H}), 7.57$ (ddd, $J=0.8,0.8,8.8 \mathrm{~Hz}, 1 \mathrm{H}), 7.49$ (ddd, $J=1.2,7.6,8.8 \mathrm{~Hz}$, $1 \mathrm{H}), 7.38-7.34(\mathrm{~m}, 2 \mathrm{H})$;

${ }^{13} \mathrm{C}\left\{{ }^{1} \mathrm{H}\right\} \mathrm{NMR}\left(\mathrm{CDCl}_{3}, 100 \mathrm{MHz}\right): \delta 156.3,155.6,135.6,129.6,127.9,126.9,123.1,122.9,120.8$, 113.7, 111.8, 85.7;

IR $\left(\mathrm{cm}^{-1}\right)$ 3044, 2924, 1462, 1435, 1414, 1269, 1244, 1202, 1018, 881, 808, 766, 752, 723, 677;

HRMS (EI) $m / z$ : [M] $]^{+}$calcd for $\mathrm{C}_{12} \mathrm{H}_{7} \mathrm{IO}, 293.9542$; found, 293.9547.

\section{2-(Thiophen-2-yl)dibenzo[b,d]furan (2s)}<smiles>c1csc(-c2ccc3oc4ccccc4c3c2)c1</smiles>

Purified by silica-gel column chromatography (hexane only);

Yield: 59.7\% (24.2 mg, $96.7 \mu \mathrm{mol})$;

pale yellow solid;

mp 91-92 ${ }^{\circ} \mathrm{C}$;

TLC $R_{\mathrm{f}} 0.65(n$-hexane/EtOAc $=10 / 1)$;

${ }^{1} \mathrm{H} \mathrm{NMR}\left(\mathrm{CDCl}_{3}, 400 \mathrm{MHz}\right): \delta 8.17(\mathrm{~d}, J=2.0 \mathrm{~Hz}, 1 \mathrm{H}), 8.00(\mathrm{dd}, J=1.2,8.0 \mathrm{~Hz}, 1 \mathrm{H}), 7.72(\mathrm{dd}, J=$ $2.0,8.8 \mathrm{~Hz}, 1 \mathrm{H}), 7.58(\mathrm{~d}, J=8.0 \mathrm{~Hz}, 1 \mathrm{H}), 7.57(\mathrm{~d}, J=8.8 \mathrm{~Hz}, 1 \mathrm{H}), 7.48(\mathrm{ddd}, J=1.2,8.0,8.0 \mathrm{~Hz}$, $1 \mathrm{H}), 7.39-7.34(\mathrm{~m}, 2 \mathrm{H}), 7.30(\mathrm{dd}, J=0.8,5.2 \mathrm{~Hz}, 1 \mathrm{H}), 7.12(\mathrm{dd}, J=3.6,5.2 \mathrm{~Hz}, 1 \mathrm{H})$;

${ }^{13} \mathrm{C}\left\{{ }^{1} \mathrm{H}\right\} \mathrm{NMR}\left(\mathrm{CDCl}_{3}, 100 \mathrm{MHz}\right): \delta 156.7,155.7,144.5,129.6,128.1,127.5,125.6,124.8,124.6$, 124.0, 123.0, 122.9, 120.8, 118.1, 111.9, 111.8;

IR $\left(\mathrm{cm}^{-1}\right)$ 3105, 3090, 3067, 3046, 1476, 1450, 1425, 1279, 1244, 1198, 1182, 1150, 1125, 1103 , $1020,880,860,843,804,766,752,727,694,687$;

HRMS (EI) $m / z$ : [M] $]^{+}$calcd for $\mathrm{C}_{16} \mathrm{H}_{10} \mathrm{OS}, 250.0452$; found, 250.0457 .

\section{(Dibenzo[b,d]furan-2-ylethynyl)trimethylsilane (2t)}

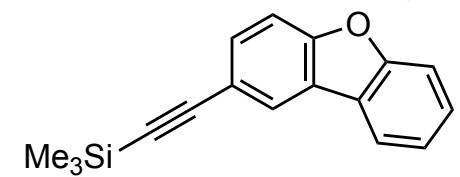

Purified by silica-gel column chromatography (hexane only);

Yield: 50.3\% (13.3 mg, $50.3 \mu \mathrm{mol})$;

colorless solid;

mp $102-103{ }^{\circ} \mathrm{C}$;

TLC $R_{\mathrm{f}} 0.70(n$-hexane/EtOAc $=20 / 1)$; 
${ }^{1} \mathrm{H}$ NMR $\left(\mathrm{CDCl}_{3}, 400 \mathrm{MHz}\right): \delta 8.09(\mathrm{dd}, J=0.8,2.0 \mathrm{~Hz}, 1 \mathrm{H}), 7.92(\mathrm{ddd}, J=0.8,1.2,7.6 \mathrm{~Hz}, 1 \mathrm{H})$, 7.59-7.55 (m, 2H), 7.51-7.45 (m, 2H), 7.36 (ddd, $J=0.8,7.6,7.6 \mathrm{~Hz}, 1 \mathrm{H}), 0.28(\mathrm{~s}, 9 \mathrm{H})$;

${ }^{13} \mathrm{C}\left\{{ }^{1} \mathrm{H}\right\} \mathrm{NMR}\left(\mathrm{CDCl}_{3}, 100 \mathrm{MHz}\right): \delta 156.6,155.9,131.2,127.6,124.6,124.3,123.6,123.1,120.8$, 117.6, 111.8, 111.7, 105.2, 93.1, $0.03(3 \mathrm{C})$;

IR $\left(\mathrm{cm}^{-1}\right) 2957,2154,1474,1449,1246,1198,1169,1123,895,839,820,756,745,727,692$;

HRMS (EI) $m / z$ : [M] $]^{+}$calcd for $\mathrm{C}_{17} \mathrm{H}_{16} \mathrm{OSi}$, 264.0970; found, 264.0980.

(17ß)-17-[(tert-Butyldimethyl)silyloxy]-2-\{(1H-naphtho[1,8-de]-1,3,2-diazaborin-2(3H)yl)phenyl\}-estra-1,3,5(10)-triene-3-ol trifluoromethanesulfonate (7)

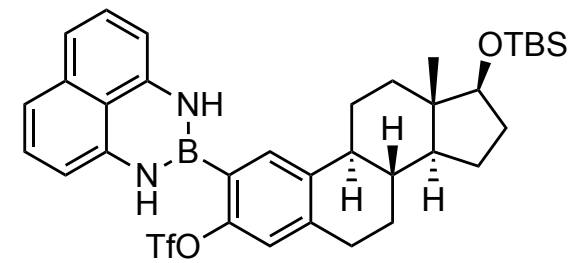

Pale gray solid;

$\operatorname{mp} 219-220^{\circ} \mathrm{C}$;

TLC $R_{\mathrm{f}} 0.55(n$-hexane/EtOAc $=5 / 1)$;

${ }^{1} \mathrm{H}$ NMR $\left(\mathrm{CDCl}_{3}, 400 \mathrm{MHz}\right): \delta 7.53(\mathrm{~s}, 1 \mathrm{H}), 7.13(\mathrm{dd}, J=7.6,7.6 \mathrm{~Hz}, 2 \mathrm{H}), 7.06(\mathrm{dd}, J=0.8,7 . \mathrm{Hz}$, 2H), $7.02(\mathrm{~s}, 1 \mathrm{H}), 6.40(\mathrm{dd}, J=1.8,7.6 \mathrm{~Hz}, 2 \mathrm{H}), 6.04(\mathrm{br} \mathrm{s}, 2 \mathrm{H}), 3.66(\mathrm{t}, J=8.0 \mathrm{~Hz}, 1 \mathrm{H}), 2.94-2.88$ (m, 2H), 2.43-2.35 (m, 1H), 2.28-2.19 (m, 1H), 2.01-1.89 (m, 3H), 1.72-1.00 (m, 8H), $0.90(\mathrm{~s}, 9 \mathrm{H})$, $0.77(\mathrm{~s}, 3 \mathrm{H}), 0.05(\mathrm{~s}, 3 \mathrm{H}), 0.03(\mathrm{~s}, 3 \mathrm{H})$;

${ }^{13} \mathrm{C}\left\{{ }^{1} \mathrm{H}\right\} \mathrm{NMR}\left(\mathrm{CDCl}_{3}, 100 \mathrm{MHz}\right): \delta 150.3,141.7,141.0,140.6(2 \mathrm{C}), 136.3,131.2,127.6(2 \mathrm{C}), 121.4$, $119.9,118.6\left(\mathrm{q}, J_{\mathrm{C}-\mathrm{F}}=321.9 \mathrm{~Hz}\right), 118.1(2 \mathrm{C}), 106.2(2 \mathrm{C}), 81.6,49.7,44.2,43.5,38.4,37.0,30.9$, 29.6, 26.7, 26.3, 25.9 (3C), 23.3, 18.1, 11.3, -4.5, -4.8 (the signal for the carbon attached to the boron atom was not observed);

${ }^{19} \mathrm{~F} \mathrm{NMR}\left(\mathrm{CDCl}_{3}, 372.5 \mathrm{MHz}\right): \delta-73.6(\mathrm{~s})$;

${ }^{11} \mathrm{~B} \mathrm{NMR}\left(\mathrm{CDCl}_{3}, 127 \mathrm{MHz}\right): \delta 28.4$;

IR $\left(\mathrm{cm}^{-1}\right)$ 2951, 2930, 2857, 2311, 1744, 1599, 1518, 1485, 1412, 1373, 1242, 1211, 1136, 1109, 891, 833, 818, 762;

HRMS (EI) $m / z$ : [M] $]^{+}$calcd for $\mathrm{C}_{35} \mathrm{H}_{44} \mathrm{BF}_{3} \mathrm{~N}_{2} \mathrm{O}_{4} \mathrm{SSi}$, 684.2836; found, 684.2831.

\section{(17ß)-17-[(tert-Butyldimethyl)silyloxy]-estra-1(10),2,4-trieno[3,2-e][1,2]benzoxaborin (1u)}<smiles></smiles>

Colorless solid;

mp $199-202{ }^{\circ} \mathrm{C}$;

TLC $R_{\mathrm{f}} 0.45(n$-hexane/EtOAc $=5 / 1)$;

${ }^{1} \mathrm{H} \mathrm{NMR}\left(\mathrm{CDCl}_{3}, 400 \mathrm{MHz}\right): \delta 8.12(\mathrm{~d}, J=8.4 \mathrm{~Hz}, 1 \mathrm{H}), 8.06-8.01(\mathrm{~m}, 2 \mathrm{H}), 7.68(\mathrm{ddd}, J=1.2,8.4$, $8.4 \mathrm{~Hz}, 1 \mathrm{H}), 7.42(\mathrm{dd}, J=8.4,8.4 \mathrm{~Hz}, 1 \mathrm{H}), 6.97(\mathrm{~s}, 1 \mathrm{H}), 4.64(\mathrm{~s}, 1 \mathrm{H}), 3.67(\mathrm{t}, J=8.4 \mathrm{~Hz}, 1 \mathrm{H}), 2.92$ $(\mathrm{dd}, J=4.0,8.8 \mathrm{~Hz}, 2 \mathrm{H}), 2.56-2.45(\mathrm{~m}, 1 \mathrm{H}), 2.35-2.24(\mathrm{~m}, 1 \mathrm{H}), 2.04-1.88(\mathrm{~m}, 3 \mathrm{H}), 1.76-0.90(\mathrm{~m}$, $8 \mathrm{H}), 0.91(\mathrm{~s}, 9 \mathrm{H}), 0.77(\mathrm{~s}, 3 \mathrm{H}), 0.06(\mathrm{~s}, 3 \mathrm{H}), 0.04(\mathrm{~s}, 3 \mathrm{H})$;

${ }^{13} \mathrm{C}\left\{{ }^{1} \mathrm{H}\right\} \mathrm{NMR}\left(\mathrm{CDCl}_{3}, 100 \mathrm{MHz}\right): \delta 149.1,140.8,138.5,135.0,133.3,132.4,126.6,121.2,120.3$, 
$120.1,119.0,81.8,49.8,44.3,43.6,38.8,37.1,31.0,29.4,27.2,26.6,25.9$ (3C), 23.3, 18.1, 11.4, $-4.5,-4.8$ (the signal for the carbon attached to the boron atom was not observed);

${ }^{11} \mathrm{~B}$ NMR $\left(\mathrm{CDCl}_{3}, 127 \mathrm{MHz}\right): \delta 27.5$;

IR $\left(\mathrm{cm}^{-1}\right)$ 2953, 2928, 2857, 1607, 1485, 1447, 1433, 1373, 1319, 1296, 1252, 1136, 1096, 1080, 881, $835,775,735$;

HRMS $\left(\mathrm{ESI}^{-}\right) \mathrm{m} / z$ : $[\mathrm{M}-\mathrm{H}]^{-}$calcd for $\mathrm{C}_{30} \mathrm{H}_{40} \mathrm{BO}_{3} \mathrm{Si}$, 487.2840; found, 487.2841.

(17ß)-17-[(tert-Butyldimethyl)silyloxy]-estra-1(10),2,4-trieno[2,3-e][1,2]benzoxaborin (1v)

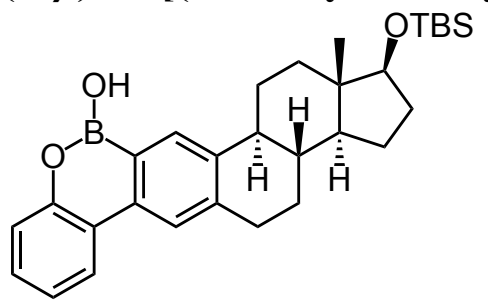

Pale gray solid;

mp $180-182^{\circ} \mathrm{C}$;

TLC $R_{\mathrm{f}} 0.75(n$-hexane/EtOAc $=10 / 1)$;

${ }^{1} \mathrm{H} \mathrm{NMR}\left(\mathrm{CDCl}_{3}, 400 \mathrm{MHz}\right): \delta 8.09(\mathrm{~d}, J=8.0 \mathrm{~Hz}, 1 \mathrm{H}), 7.97(\mathrm{~s}, 1 \mathrm{H}), 7.86(\mathrm{~s}, 1 \mathrm{H}), 7.33(\mathrm{dd}, J=7.2$ $\mathrm{Hz}, 1 \mathrm{H}), 7.28-7.15(\mathrm{~m}, 2 \mathrm{H}), 4.64(\mathrm{~s}, 1 \mathrm{H}), 3.66(\mathrm{t}, J=8.0 \mathrm{~Hz}, 1 \mathrm{H}), 3.07-3.01(\mathrm{~m}, 2 \mathrm{H}), 2.49(\mathrm{~m}, 1 \mathrm{H})$, 2.36-2.26 (m, 1H), 2.00-1.89 (m, 3H), 1.73-1.14 (m, 8H), $0.90(\mathrm{~s}, 9 \mathrm{H}), 0.76(\mathrm{~s}, 3 \mathrm{H}), 0.05(\mathrm{~s}, 3 \mathrm{H})$, $0.04(\mathrm{~s}, 3 \mathrm{H})$;

${ }^{13} \mathrm{C}\left\{{ }^{1} \mathrm{H}\right\}$ NMR $\left(\mathrm{CDCl}_{3}, 100 \mathrm{MHz}\right): \delta 151.0,142.1,140.3,137.7,130.0,128.4,123.4,123.0,122.5$, $121.9,119.4,81.7,49.9,44.5,43.6,38.6,37.1,31.0,30.3,27.1,26.2,25.9$ (3C), 23.3, 18.1, 11.3, $-4.5,-4.8$ (the signal for the carbon attached to the boron atom was not observed);

${ }^{11} \mathrm{~B}$ NMR $\left(\mathrm{CDCl}_{3}, 127 \mathrm{MHz}\right): \delta 27.3$;

IR $\left(\mathrm{cm}^{-1}\right)$ 3566, 2951, 2926, 2855, 1612, 1541, 1501, 1487, 1472, 1452, 1412, 1383, 1329, 1315, $1288,1281,1248,1198,1140,1096,905,872,835,814,773,748,727,692,669$;

HRMS $\left(\mathrm{ESI}^{-}\right) \mathrm{m} / z$ : $[\mathrm{M}-\mathrm{H}]^{-}$calcd for $\mathrm{C}_{30} \mathrm{H}_{40} \mathrm{BO}_{3} \mathrm{Si}$, 487.2840; found, 487.2845.

(17ß)-17-[(tert-Butyldimethyl)silyloxy]-estra-1(10),2,4-trieno[3,2-b][1,2]benzofuran (2u)

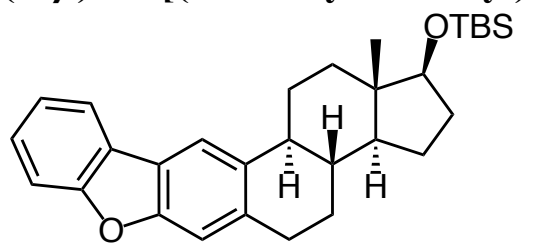

Purified by silica-gel column chromatography (hexane only);

Yield: $85.5 \%$ (39.4 mg, $85.5 \mu \mathrm{mol})$;

colorless solid;

mp $170-172{ }^{\circ} \mathrm{C}$;

TLC $R_{\mathrm{f}} 0.80(n$-hexane/EtOAc $=20 / 1)$;

${ }^{1} \mathrm{H} \mathrm{NMR}\left(\mathrm{CDCl}_{3}, 400 \mathrm{MHz}\right): \delta 7.90(\mathrm{dd}, J=0.8,7.6 \mathrm{~Hz}, 1 \mathrm{H}), 7.86(\mathrm{~s}, 1 \mathrm{H}), 7.51(\mathrm{~d}, J=7.6 \mathrm{~Hz}, 1 \mathrm{H})$, 7.40 (ddd, $J=0.8,7.6,7.6 \mathrm{~Hz}, 1 \mathrm{H}), 7.30$ (ddd, $J=0.8,7.6,7.6 \mathrm{~Hz}, 1 \mathrm{H}), 7.26(\mathrm{~s}, 1 \mathrm{H}), 3.68(\mathrm{t}, J=8.4$ $\mathrm{Hz}, 1 \mathrm{H}), 3.07-3.01(\mathrm{~m}, 2 \mathrm{H}), 2.52-2.45(\mathrm{~m}, 1 \mathrm{H}), 2.39-2.31(\mathrm{~m}, 1 \mathrm{H}), 2.01-1.90(\mathrm{~m}, 3 \mathrm{H}), 1.74-1.16$ $(\mathrm{m}, 8 \mathrm{H}), 0.91(\mathrm{~s}, 9 \mathrm{H}), 0.78(\mathrm{~s}, 3 \mathrm{H}), 0.06(\mathrm{~s}, 3 \mathrm{H}), 0.04(\mathrm{~s}, 3 \mathrm{H})$;

${ }^{13} \mathrm{C}\left\{{ }^{1} \mathrm{H}\right\}$ NMR $\left(\mathrm{CDCl}_{3}, 100 \mathrm{MHz}\right): \delta 156.3,154.7,137.0,135.7,126.4,124.6,122.4,121.9,120.2$, 116.9, 111.5, 111.0, 81.8, 49.9, 44.5, 43.6, 38.8, 37.2, 31.0, 30.4, 27.3, 26.8, 25.9 (3C), 23.4, 18.1, 
$11.4,-4.4,-4.8$;

IR $\left(\mathrm{cm}^{-1}\right) 2951,2926,2855,1464,1454,1429,1360,1248,1202,1140,1123,1092,1078,1013,908$, $885,858,835,816,773,745,733,667$;

HRMS (EI) $m / z:[\mathrm{M}]^{+}$calcd for $\mathrm{C}_{30} \mathrm{H}_{40} \mathrm{O}_{2} \mathrm{Si}$, 460.2798; found, 460.2802.

(17ß)-17-[(tert-Butyldimethyl)silyloxy]-estra-1(10),2,4-trieno[3,2-b][1,2]benzofuran (2v)

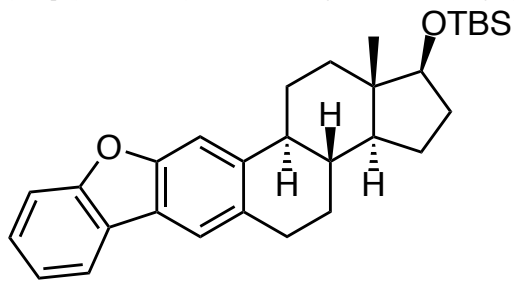

Purified by silica-gel column chromatography (hexane only);

Yield: $43.2 \%$ (17.5 mg, $38.0 \mu \mathrm{mol})$;

colorless solid;

mp $166-168^{\circ} \mathrm{C}$;

TLC $R_{\mathrm{f}} 0.30(n$-hexane/EtOAc $=10 / 1)$;

${ }^{1} \mathrm{H} \mathrm{NMR}\left(\mathrm{CDCl}_{3}, 400 \mathrm{MHz}\right): \delta 7.88(\mathrm{ddd}, J=1.2,1.2,7.6 \mathrm{~Hz}, 1 \mathrm{H}), 7.64(\mathrm{~s}, 1 \mathrm{H}), 7.53-7.50(\mathrm{~m}, 2 \mathrm{H})$, 7.40 (ddd, $J=1.2,7.6,7.6 \mathrm{~Hz}, 1 \mathrm{H}), 7.30$ (ddd, $J=1.2,7.6,7.6 \mathrm{~Hz}, 1 \mathrm{H}), 3.67$ (t, $J=8.0 \mathrm{~Hz}, 1 \mathrm{H}$ ), 3.06-3.01 (m, 2H), 2.42-2.33 (m, 2H), 2.00-1.91 (m, 3H), 1.74-1.16 (m, 8H), $0.91(\mathrm{~s}, 9 \mathrm{H}), 0.78(\mathrm{~s}$, $3 \mathrm{H}), 0.06(\mathrm{~s}, 3 \mathrm{H}), 0.04(\mathrm{~s}, 3 \mathrm{H})$;

${ }^{13} \mathrm{C}\left\{{ }^{1} \mathrm{H}\right\}$ NMR $\left(\mathrm{CDCl}_{3}, 100 \mathrm{MHz}\right): \delta 156.4,155.2,140.8,131.5,126.5,124.3,122.4,121.7,120.3$, 120.1, 111.5, 108.0, 81.7, 50.0, 45.1, 43.6, 38.6, 37.1, 31.0, 29.6, 27.4, 26.5, 25.9 (3C), 23.4, 18.1, $11.4,-4.4,-4.8$;

IR $\left(\mathrm{cm}^{-1}\right)$ 2953, 2928, 2855, 1362, 1250, 1206, 1142, 835, 773, 746;

HRMS (EI) $m / z$ : [M] $]^{+}$calcd for $\mathrm{C}_{30} \mathrm{H}_{40} \mathrm{O}_{2} \mathrm{Si}$, 460.2798; found, 460.2785. 


\section{References for Supporting Information}

(S1) Sumida, Y.; Harada, R.; Kato-Sumida, T.; Johmoto, K.; Uekusa, H.; Hosoya, T. Org. Lett. 2014, 16, 6240 .

(S2) Tomsho, J. W.; Pal, A.; Hall, D. G.; Benkovic, S. J. ACS Med. Chem. Lett. 2012, 3, 48.

(S3) Sumida, Y.; Kato, T.; Hosoya, T. Org. Lett. 2013, 15, 2806.

(S4) Wood, J. L.; Marciasini, L. D.; Vaultier, M.; Pucheault, M. Synlett 2014, 25, 551.

(S5) Wang, C.; Piel, I.; Glorius, F. J. Am. Chem. Soc. 2009, 131, 4194.

(S6) Liu, Z.; Larock, R. C. Org. Lett. 2004, 6, 3739.

(S7) Lockner, J. W.; Dixon, D. D.; Risgaard, R.; Baran, P. S. Org. Lett. 2011, 13, 5628.

(S8) Xiao, B.; Gong, T.-J.; Liu, Z.-J.; Liu, J.-H.; Luo, D.-F.; Xu, J.; Liu, L. J. Am. Chem. Soc. 2011, 133, 9250 .

(S9) Wei, Y.; Yoshikai, N. Org. Lett. 2011, 13, 5504.

(S10) Ferguson, D. M.; Rudolph, S. R.; Kalyani, D. ACS Catal. 2014, 4, 2395.

(S11) Yue, W. S.; Li, J. J. Org. Lett. 2002, 4, 2201.

(S12) Maetani, S.; Fukuyama, T.; Ryu, I. Org. Lett. 2013, 15, 2754. 


\section{Compound 1q}

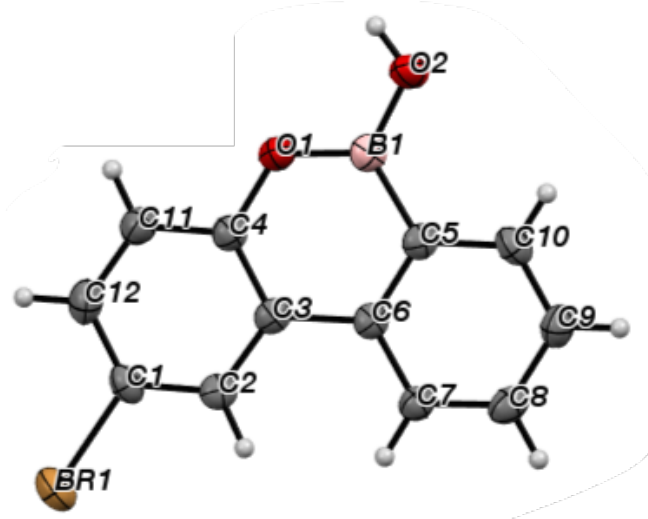

Figure S1. ORTEP diagram of the X-ray structure of 1q. Ellipsoids are drawn at 50\% probability.

Table S5. Crystal data and structure refinement for $\mathbf{1 q}$.

Identification code

Empirical formula

Formula weight

Temperature

Wavelength

Crystal system

Space group

Unit cell dimensions

Volume

Z

Density (calculated)

Absorption coefficient

$\mathrm{F}(000)$

Crystal size

Theta range for data collection

Index ranges

Reflections collected

Independent reflections

Completeness to theta $=27.48^{\circ}$

Absorption correction

Max. and min. transmission

Refinement method

Data / restraints / parameters

Goodness-of-fit on $F^{2}$

Final $R$ indices [I $>2 \operatorname{sigma}(\mathrm{I})]$

$R$ indices (all data)

Largest diff. peak and hole compound $\mathbf{1 q}$

$\mathrm{C}_{12} \mathrm{H}_{8} \mathrm{BBrO}_{2}$

274.91

293(2) K

$0.71075 \AA$

Triclinic

$\mathrm{P}-1$

$a=4.8437(7) \AA$

$\alpha=64.935(5)^{\circ}$.

$b=15.386(2) \AA$

$c=16.179(2) \AA$

1082.9(2) $\AA^{3}$

4

$1.686 \mathrm{Mg} / \mathrm{m}^{3}$

$3.773 \mathrm{~mm}^{-1}$

\section{4}

$0.44 \times 0.34 \times 0.08 \mathrm{~mm}^{3}$

3.09 to $27.48^{\circ}$.

$-6<=h<=6,-19<=k<=19,-20<=l<=20$

11236

$4958[R($ int $)=0.0599]$

$99.7 \%$

Semi-empirical from equivalents

0.7548 and 0.4775

Full-matrix least-squares on $F^{2}$

4958 / 0 / 295

1.045

$R_{1}=0.0518, w R_{2}=0.1023$

$R_{1}=0.0797, w R_{2}=0.1135$

0.786 and -0.817 e. $\AA^{-3}$ 
Table S6. Atomic coordinates $\left(\times 10^{4}\right)$ and equivalent isotropic displacement parameters $\left(\AA^{2} \times 10^{3}\right)$ for 1q. $U$ (eq) is defined as one third of the trace of the orthogonalized $U^{\mathrm{ij}}$ tensor.

\begin{tabular}{|c|c|c|c|c|}
\hline & $\mathrm{x}$ & $\mathrm{y}$ & $\mathrm{z}$ & $\mathrm{U}(\mathrm{eq})$ \\
\hline $\operatorname{Br}(1)$ & $-3205(1)$ & $5770(1)$ & $1576(1)$ & $41(1)$ \\
\hline $\mathrm{O}(1)$ & $4812(5)$ & $2342(2)$ & $3795(2)$ & $34(1)$ \\
\hline $\mathrm{O}(2)$ & $8658(6)$ & $1189(2)$ & $4019(2)$ & $40(1)$ \\
\hline$C(1)$ & $-674(7)$ & 4681(3) & $2265(3)$ & $30(1)$ \\
\hline$C(2)$ & 1341(7) & $4319(3)$ & 1814(3) & $28(1)$ \\
\hline$C(3)$ & $3274(7)$ & $3536(3)$ & $2296(2)$ & $26(1)$ \\
\hline$C(4)$ & $3033(8)$ & $3131(3)$ & $3251(2)$ & $30(1)$ \\
\hline$C(5)$ & $7351(7)$ & $2345(3)$ & $2383(2)$ & $27(1)$ \\
\hline$C(6)$ & $5517(7)$ & $3143(3)$ & $1844(2)$ & $26(1)$ \\
\hline$C(7)$ & $5890(8)$ & $3556(3)$ & $889(2)$ & $31(1)$ \\
\hline $\mathrm{C}(8)$ & $8069(8)$ & $3180(3)$ & 497(3) & $36(1)$ \\
\hline C(9) & $9888(8)$ & 2393(3) & 1029(3) & $36(1)$ \\
\hline$C(10)$ & $9524(8)$ & 1983(3) & 1958(3) & $33(1)$ \\
\hline $\mathrm{C}(11)$ & $1010(8)$ & $3490(3)$ & $3694(3)$ & $37(1)$ \\
\hline$C(12)$ & $-876(8)$ & $4277(3)$ & $3204(3)$ & $40(1)$ \\
\hline $\mathrm{B}(1)$ & $6953(9)$ & 1941(3) & $3428(3)$ & $31(1)$ \\
\hline $\operatorname{Br}(2)$ & $7243(1)$ & $1652(1)$ & $9476(1)$ & $47(1)$ \\
\hline $\mathrm{O}(3)$ & $1758(5)$ & $367(2)$ & $7018(2)$ & $33(1)$ \\
\hline $\mathrm{O}(4)$ & $-1287(6)$ & $547(2)$ & $5884(2)$ & $39(1)$ \\
\hline$C(13)$ & $5532(8)$ & $1239(3)$ & $8690(3)$ & $32(1)$ \\
\hline $\mathrm{C}(14)$ & $3531(8)$ & 1889(3) & $8096(2)$ & $33(1)$ \\
\hline$C(15)$ & 2181(7) & 1621(3) & $7520(2)$ & $28(1)$ \\
\hline$C(16)$ & $2945(8)$ & $698(3)$ & $7569(2)$ & $29(1)$ \\
\hline $\mathrm{C}(17)$ & $-1257(8)$ & $1960(3)$ & $6317(2)$ & $29(1)$ \\
\hline $\mathrm{C}(18)$ & $22(7)$ & $2284(3)$ & $6874(2)$ & $29(1)$ \\
\hline C(19) & $-829(8)$ & $3215(3)$ & 6812(3) & $37(1)$ \\
\hline$C(20)$ & $-2862(9)$ & $3803(3)$ & $6214(3)$ & $42(1)$ \\
\hline $\mathrm{C}(21)$ & $-4155(9)$ & $3488(3)$ & $5666(3)$ & $42(1)$ \\
\hline $\mathrm{C}(22)$ & $-3342(8)$ & $2580(3)$ & $5718(3)$ & $34(1)$ \\
\hline $\mathrm{C}(23)$ & $4960(8)$ & $58(3)$ & $8165(3)$ & $35(1)$ \\
\hline $\mathrm{C}(24)$ & $6274(8)$ & $329(3)$ & $8738(3)$ & $38(1)$ \\
\hline $\mathrm{B}(2)$ & $-269(9)$ & $938(3)$ & $6399(3)$ & $30(1)$ \\
\hline
\end{tabular}


Table S7. Bond lengths $[\AA]$ and angles $\left[^{\circ}\right]$ for $\mathbf{1 q}$.

\begin{tabular}{|c|c|}
\hline $\mathrm{Br}(1)-\mathrm{C}(1)$ & $1.901(4)$ \\
\hline $\mathrm{O}(1)-\mathrm{B}(1)$ & $1.368(5)$ \\
\hline $\mathrm{O}(1)-\mathrm{C}(4)$ & $1.383(4)$ \\
\hline $\mathrm{O}(2)-\mathrm{B}(1)$ & $1.361(5)$ \\
\hline $\mathrm{O}(2)-\mathrm{H}(1)$ & $0.85(4)$ \\
\hline $\mathrm{C}(1)-\mathrm{C}(12)$ & $1.378(5)$ \\
\hline $\mathrm{C}(1)-\mathrm{C}(2)$ & $1.380(5)$ \\
\hline$C(2)-C(3)$ & $1.393(5)$ \\
\hline $\mathrm{C}(2)-\mathrm{H}(2)$ & 0.9300 \\
\hline$C(3)-C(4)$ & $1.403(5)$ \\
\hline C(3)-C(6) & $1.476(5)$ \\
\hline $\mathrm{C}(4)-\mathrm{C}(11)$ & $1.373(5)$ \\
\hline$C(5)-C(10)$ & $1.403(5)$ \\
\hline C(5)-C(6) & $1.403(5)$ \\
\hline $\mathrm{C}(5)-\mathrm{B}(1)$ & $1.542(6)$ \\
\hline$C(6)-C(7)$ & $1.410(5)$ \\
\hline$C(7)-C(8)$ & $1.384(5)$ \\
\hline $\mathrm{C}(7)-\mathrm{H}(7)$ & 0.9300 \\
\hline $\mathrm{C}(8)-\mathrm{C}(9)$ & $1.387(5)$ \\
\hline $\mathrm{C}(8)-\mathrm{H}(8)$ & 0.9300 \\
\hline$C(9)-C(10)$ & $1.370(5)$ \\
\hline $\mathrm{C}(9)-\mathrm{H}(9)$ & 0.9300 \\
\hline $\mathrm{C}(10)-\mathrm{H}(10)$ & 0.9300 \\
\hline$C(11)-C(12)$ & $1.387(5)$ \\
\hline $\mathrm{C}(11)-\mathrm{H}(11)$ & 0.9300 \\
\hline $\mathrm{C}(12)-\mathrm{H}(12)$ & 0.9300 \\
\hline $\operatorname{Br}(2)-C(13)$ & $1.914(4)$ \\
\hline $\mathrm{O}(3)-\mathrm{B}(2)$ & $1.377(5)$ \\
\hline $\mathrm{O}(3)-\mathrm{C}(16)$ & $1.383(4)$ \\
\hline $\mathrm{O}(4)-\mathrm{B}(2)$ & $1.363(5)$ \\
\hline $\mathrm{O}(4)-\mathrm{H}(4)$ & $0.83(5)$ \\
\hline$C(13)-C(24)$ & $1.371(6)$ \\
\hline $\mathrm{C}(13)-\mathrm{C}(14)$ & $1.386(5)$ \\
\hline$C(14)-C(15)$ & $1.392(5)$ \\
\hline $\mathrm{C}(14)-\mathrm{H}(14)$ & 0.9300 \\
\hline$C(15)-C(16)$ & $1.391(5)$ \\
\hline $\mathrm{C}(15)-\mathrm{C}(18)$ & $1.480(5)$ \\
\hline$C(16)-C(23)$ & $1.385(5)$ \\
\hline $\mathrm{C}(17)-\mathrm{C}(22)$ & $1.401(5)$ \\
\hline $\mathrm{C}(17)-\mathrm{C}(18)$ & $1.407(5)$ \\
\hline $\mathrm{C}(17)-\mathrm{B}(2)$ & $1.533(6)$ \\
\hline $\mathrm{C}(18)-\mathrm{C}(19)$ & $1.400(5)$ \\
\hline $\mathrm{C}(19)-\mathrm{C}(20)$ & $1.370(5)$ \\
\hline C(19)-H(19) & 0.9300 \\
\hline $\mathrm{C}(20)-\mathrm{C}(21)$ & $1.389(6)$ \\
\hline
\end{tabular}




$\begin{array}{ll}\mathrm{C}(20)-\mathrm{H}(20) & \\ \mathrm{C}(21)-\mathrm{C}(22) & 1.370(6) \\ \mathrm{C}(21)-\mathrm{H}(21) & 0.9300 \\ \mathrm{C}(22)-\mathrm{H}(22) & 0.9300 \\ \mathrm{C}(23)-\mathrm{C}(24) & 1.382(5) \\ \mathrm{C}(23)-\mathrm{H}(23) & 0.9300 \\ \mathrm{C}(24)-\mathrm{H}(24) & 0.9300 \\ & \\ \mathrm{~B}(1)-\mathrm{O}(1)-\mathrm{C}(4) & 121.5(3) \\ \mathrm{B}(1)-\mathrm{O}(2)-\mathrm{H}(1) & 117(3) \\ \mathrm{C}(12)-\mathrm{C}(1)-\mathrm{C}(2) & 121.5(4) \\ \mathrm{C}(12)-\mathrm{C}(1)-\mathrm{B}(1) & 119.2(3) \\ \mathrm{C}(2)-\mathrm{C}(1)-\mathrm{B}(1) & 119.2(3) \\ \mathrm{C}(1)-\mathrm{C}(2)-\mathrm{C}(3) & 121.0(3) \\ \mathrm{C}(1)-\mathrm{C}(2)-\mathrm{H}(2) & 119.5 \\ \mathrm{C}(3)-\mathrm{C}(2)-\mathrm{H}(2) & 119.5 \\ \mathrm{C}(2)-\mathrm{C}(3)-\mathrm{C}(4) & 116.8(3) \\ \mathrm{C}(2)-\mathrm{C}(3)-\mathrm{C}(6) & 122.9(3) \\ \mathrm{C}(4)-\mathrm{C}(3)-\mathrm{C}(6) & 120.3(3) \\ \mathrm{C}(11)-\mathrm{C}(4)-\mathrm{O}(1) & 116.4(3) \\ \mathrm{C}(11)-\mathrm{C}(4)-\mathrm{C}(3) & 121.9(3) \\ \mathrm{O}(1)-\mathrm{C}(4)-\mathrm{C}(3) & 121.7(3) \\ \mathrm{C}(10)-\mathrm{C}(5)-\mathrm{C}(6) & 119.2(3) \\ \mathrm{C}(10)-\mathrm{C}(5)-\mathrm{B}(1) & 123.2(3) \\ \mathrm{C}(6)-\mathrm{C}(5)-\mathrm{B}(1) & 117.6(3) \\ \mathrm{C}(5)-\mathrm{C}(6)-\mathrm{C}(7) & 119.1(3) \\ \mathrm{C}(5)-\mathrm{C}(6)-\mathrm{C}(3) & 119.0(3) \\ \mathrm{C}(7)-\mathrm{C}(6)-\mathrm{C}(3) & 121.9(3) \\ \mathrm{C}(8)-\mathrm{C}(7)-\mathrm{C}(6) & 119.9(4) \\ \mathrm{C}(8)-\mathrm{C}(7)-\mathrm{H}(7) & 120.1 \\ \mathrm{C}(6)-\mathrm{C}(7)-\mathrm{H}(7) & 120.1 \\ \mathrm{C}(7)-\mathrm{C}(8)-\mathrm{C}(9) & 121.1(4) \\ \mathrm{C}(7)-\mathrm{C}(8)-\mathrm{H}(8) & 119.5 \\ \mathrm{C}(9)-\mathrm{C}(8)-\mathrm{H}(8) & 119.5 \\ \mathrm{C}(10)-\mathrm{C}(9)-\mathrm{C}(8) & 119.4(4) \\ \mathrm{C}(10)-\mathrm{C}(9)-\mathrm{H}(9) & 120.3 \\ \mathrm{C}(8)-\mathrm{C}(9)-\mathrm{H}(9) & 120.3 \\ \mathrm{C}(9)-\mathrm{C}(10)-\mathrm{C}(5) & 121.4(4) \\ \mathrm{C}(9)-\mathrm{C}(10)-\mathrm{H}(10) & 119.3 \\ \mathrm{C}(5)-\mathrm{C}(10)-\mathrm{H}(10) & 119.3 \\ \mathrm{C}(4)-\mathrm{C}(11)-\mathrm{C}(12) & 120.5(4) \\ \mathrm{C}(4)-\mathrm{C}(11)-\mathrm{H}(11) & 119.8 \\ \mathrm{C}(12)-\mathrm{C}(11)-\mathrm{H}(11) & 119.8 \\ \mathrm{C}(1)-\mathrm{C}(12)-\mathrm{C}(11) & 118.3(4) \\ \mathrm{C}(1)-\mathrm{C}(12)-\mathrm{H}(12) & 120.8 \\ \mathrm{C}(11)-\mathrm{C}(12)-\mathrm{H}(12) & 120.8 \\ \mathrm{O}(2)-\mathrm{B}(1)-\mathrm{O}(1) & \\ & \\ & \end{array}$




$\begin{array}{ll}\mathrm{O}(2)-\mathrm{B}(1)-\mathrm{C}(5) & 122.8(4) \\ \mathrm{O}(1)-\mathrm{B}(1)-\mathrm{C}(5) & 119.9(3) \\ \mathrm{B}(2)-\mathrm{O}(3)-\mathrm{C}(16) & 121.3(3) \\ \mathrm{B}(2)-\mathrm{O}(4)-\mathrm{H}(4) & 115(3) \\ \mathrm{C}(24)-\mathrm{C}(13)-\mathrm{C}(14) & 122.6(4) \\ \mathrm{C}(24)-\mathrm{C}(13)-\mathrm{Br}(2) & 120.1(3) \\ \mathrm{C}(14)-\mathrm{C}(13)-\mathrm{Br}(2) & 117.3(3) \\ \mathrm{C}(13)-\mathrm{C}(14)-\mathrm{C}(15) & 119.8(4) \\ \mathrm{C}(13)-\mathrm{C}(14)-\mathrm{H}(14) & 120.1 \\ \mathrm{C}(15)-\mathrm{C}(14)-\mathrm{H}(14) & 120.1 \\ \mathrm{C}(16)-\mathrm{C}(15)-\mathrm{C}(14) & 117.4(3) \\ \mathrm{C}(16)-\mathrm{C}(15)-\mathrm{C}(18) & 120.5(3) \\ \mathrm{C}(14)-\mathrm{C}(15)-\mathrm{C}(18) & 122.1(4) \\ \mathrm{O}(3)-\mathrm{C}(16)-\mathrm{C}(23) & 116.1(3) \\ \mathrm{O}(3)-\mathrm{C}(16)-\mathrm{C}(15) & 121.9(3) \\ \mathrm{C}(23)-\mathrm{C}(16)-\mathrm{C}(15) & 122.1(4) \\ \mathrm{C}(22)-\mathrm{C}(17)-\mathrm{C}(18) & 118.9(4) \\ \mathrm{C}(22)-\mathrm{C}(17)-\mathrm{B}(2) & 123.5(4) \\ \mathrm{C}(18)-\mathrm{C}(17)-\mathrm{B}(2) & 117.7(3) \\ \mathrm{C}(19)-\mathrm{C}(18)-\mathrm{C}(17) & 119.0(3) \\ \mathrm{C}(19)-\mathrm{C}(18)-\mathrm{C}(15) & 122.2(4) \\ \mathrm{C}(17)-\mathrm{C}(18)-\mathrm{C}(15) & 118.8(3) \\ \mathrm{C}(20)-\mathrm{C}(19)-\mathrm{C}(18) & 120.5(4) \\ \mathrm{C}(20)-\mathrm{C}(19)-\mathrm{H}(19) & 119.8 \\ \mathrm{C}(18)-\mathrm{C}(19)-\mathrm{H}(19) & 119.8 \\ \mathrm{C}(19)-\mathrm{C}(20)-\mathrm{C}(21) & 121.0(4) \\ \mathrm{C}(19)-\mathrm{C}(20)-\mathrm{H}(20) & 119.5 \\ \mathrm{C}(21)-\mathrm{C}(20)-\mathrm{H}(20) & 119.5 \\ \mathrm{C}(22)-\mathrm{C}(21)-\mathrm{C}(20) & 119.1(4) \\ \mathrm{C}(22)-\mathrm{C}(21)-\mathrm{H}(21) & 120.4 \\ \mathrm{C}(20)-\mathrm{C}(21)-\mathrm{H}(21) & 120.4 \\ \mathrm{C}(21)-\mathrm{C}(22)-\mathrm{C}(17) & 121.5(4) \\ \mathrm{C}(21)-\mathrm{C}(22)-\mathrm{H}(22) & 119.3 \\ \mathrm{C}(17)-\mathrm{C}(22)-\mathrm{H}(22) & 119.3 \\ \mathrm{C}(24)-\mathrm{C}(23)-\mathrm{C}(16) & 120.1(4) \\ \mathrm{C}(24)-\mathrm{C}(23)-\mathrm{H}(23) & 120.0 \\ \mathrm{C}(16)-\mathrm{C}(23)-\mathrm{H}(23) & 120.0 \\ \mathrm{C}(13)-\mathrm{C}(24)-\mathrm{C}(23) & 118.1(4) \\ \mathrm{C}(13)-\mathrm{C}(24)-\mathrm{H}(24) & 121.0 \\ \mathrm{C}(23)-\mathrm{C}(24)-\mathrm{H}(24) & 121.0 \\ \mathrm{O}(4)-\mathrm{B}(2)-\mathrm{O}(3) & 116.6(4) \\ \mathrm{O}(4)-\mathrm{B}(2)-\mathrm{C}(17) & 123.5(4) \\ \mathrm{O}(3)-\mathrm{B}(2)-\mathrm{C}(17) & 119.9(3) \\ & \end{array}$

Symmetry transformations used to generate equivalent atoms: 
Table S8. Anisotropic displacement parameters $\left(\AA^{2} \times 10^{3}\right)$ for 1q. The anisotropic displacement factor exponent takes the form: $-2 \pi^{2}\left[\mathrm{~h}^{2} \mathrm{a}^{* 2} \mathrm{U}^{11}+\ldots+2 \mathrm{~h} \mathrm{k} \mathrm{a}^{*} \mathrm{~b}^{*} \mathrm{U}^{12}\right]$

\begin{tabular}{|c|c|c|c|c|c|c|}
\hline & $\mathrm{U}^{11}$ & $\mathrm{U}^{22}$ & $\mathrm{U}^{33}$ & $\mathrm{U}^{23}$ & $\mathrm{U}^{13}$ & $\mathrm{U}^{12}$ \\
\hline $\operatorname{Br}(1)$ & $36(1)$ & $33(1)$ & $53(1)$ & $-19(1)$ & $-7(1)$ & $4(1)$ \\
\hline $\mathrm{O}(1)$ & $39(2)$ & $34(2)$ & $26(1)$ & $-12(1)$ & $-3(1)$ & 1(1) \\
\hline $\mathrm{O}(2)$ & $54(2)$ & $35(2)$ & $29(1)$ & $-16(1)$ & $-9(1)$ & $10(1)$ \\
\hline$C(1)$ & $25(2)$ & $29(2)$ & $41(2)$ & $-18(2)$ & $-5(2)$ & $-1(2)$ \\
\hline$C(2)$ & $32(2)$ & $25(2)$ & $30(2)$ & $-12(2)$ & $-1(2)$ & $-7(2)$ \\
\hline$C(3)$ & $29(2)$ & $29(2)$ & $28(2)$ & $-16(2)$ & $-2(2)$ & $-8(2)$ \\
\hline$C(4)$ & $31(2)$ & $31(2)$ & $31(2)$ & $-15(2)$ & $-2(2)$ & $-3(2)$ \\
\hline$C(5)$ & $32(2)$ & $27(2)$ & $29(2)$ & $-16(2)$ & $-2(2)$ & $-7(2)$ \\
\hline$C(6)$ & $28(2)$ & $25(2)$ & $29(2)$ & $-14(2)$ & $-1(2)$ & $-6(2)$ \\
\hline$C(7)$ & $32(2)$ & $33(2)$ & $28(2)$ & $-14(2)$ & $-1(2)$ & $-3(2)$ \\
\hline $\mathrm{C}(8)$ & $46(2)$ & $38(2)$ & $29(2)$ & $-17(2)$ & $2(2)$ & $-10(2)$ \\
\hline$C(9)$ & $39(2)$ & $42(2)$ & $37(2)$ & $-25(2)$ & $3(2)$ & $-3(2)$ \\
\hline$C(10)$ & $32(2)$ & $29(2)$ & $41(2)$ & $-18(2)$ & $-6(2)$ & $2(2)$ \\
\hline $\mathrm{C}(11)$ & $37(2)$ & $44(3)$ & $31(2)$ & $-18(2)$ & $2(2)$ & $-3(2)$ \\
\hline$C(12)$ & $32(2)$ & $49(3)$ & $44(2)$ & $-27(2)$ & $5(2)$ & $-2(2)$ \\
\hline $\mathrm{B}(1)$ & $34(2)$ & $30(2)$ & $33(2)$ & $-18(2)$ & $-4(2)$ & $-4(2)$ \\
\hline $\operatorname{Br}(2)$ & $50(1)$ & $55(1)$ & $42(1)$ & $-24(1)$ & $-6(1)$ & $-11(1)$ \\
\hline $\mathrm{O}(3)$ & $40(2)$ & $28(1)$ & $32(1)$ & $-16(1)$ & $-4(1)$ & $4(1)$ \\
\hline $\mathrm{O}(4)$ & $56(2)$ & $30(2)$ & $32(2)$ & $-16(1)$ & $-11(1)$ & $5(1)$ \\
\hline$C(13)$ & $28(2)$ & $41(2)$ & $31(2)$ & $-18(2)$ & $2(2)$ & $-6(2)$ \\
\hline$C(14)$ & $35(2)$ & $32(2)$ & $33(2)$ & $-16(2)$ & $5(2)$ & $-6(2)$ \\
\hline $\mathrm{C}(15)$ & $27(2)$ & $28(2)$ & $30(2)$ & $-13(2)$ & $4(2)$ & $-2(2)$ \\
\hline$C(16)$ & $31(2)$ & $29(2)$ & $29(2)$ & $-14(2)$ & $0(2)$ & $-3(2)$ \\
\hline$C(17)$ & $34(2)$ & $31(2)$ & $22(2)$ & $-12(2)$ & $6(2)$ & $0(2)$ \\
\hline $\mathrm{C}(18)$ & $26(2)$ & $26(2)$ & $31(2)$ & $-11(2)$ & $5(2)$ & $1(2)$ \\
\hline$C(19)$ & $38(2)$ & $30(2)$ & $40(2)$ & $-15(2)$ & $-2(2)$ & $2(2)$ \\
\hline $\mathrm{C}(20)$ & $52(3)$ & $27(2)$ & $44(2)$ & $-13(2)$ & $5(2)$ & $0(2)$ \\
\hline $\mathrm{C}(21)$ & $41(2)$ & $35(2)$ & $36(2)$ & $-7(2)$ & $-1(2)$ & $13(2)$ \\
\hline$C(22)$ & $37(2)$ & $36(2)$ & $29(2)$ & $-14(2)$ & $-1(2)$ & $1(2)$ \\
\hline$C(23)$ & $41(2)$ & $28(2)$ & $35(2)$ & $-13(2)$ & $-3(2)$ & $4(2)$ \\
\hline $\mathrm{C}(24)$ & $34(2)$ & $42(2)$ & $35(2)$ & $-14(2)$ & $-3(2)$ & $-2(2)$ \\
\hline $\mathrm{B}(2)$ & $38(2)$ & $33(2)$ & $21(2)$ & $-14(2)$ & $5(2)$ & $-6(2)$ \\
\hline
\end{tabular}


Table S9. Hydrogen coordinates $\left(\times 10^{4}\right)$ and isotropic displacement parameters $\left(\AA^{2} \times 10^{3}\right)$ for $\mathbf{1 q}$.

\begin{tabular}{lcccc}
\hline & $\mathrm{x}$ & $\mathrm{y}$ & $\mathrm{z}$ & $\mathrm{U}(\mathrm{eq})$ \\
\hline $\mathrm{H}(1)$ & $8320(90)$ & $1020(40)$ & $4580(30)$ & 59 \\
$\mathrm{H}(2)$ & 1410 & 4602 & 1180 & 34 \\
$\mathrm{H}(7)$ & 4675 & 4080 & 523 & 37 \\
$\mathrm{H}(8)$ & 8317 & 3460 & -133 & 44 \\
$\mathrm{H}(9)$ & 11342 & 2145 & 758 & 44 \\
$\mathrm{H}(10)$ & 10743 & 1454 & 2313 & 40 \\
$\mathrm{H}(11)$ & 902 & 3203 & 4328 & 44 \\
$\mathrm{H}(12)$ & -2242 & 4527 & 3502 & 47 \\
$\mathrm{H}(4)$ & $-670(100)$ & $-20(40)$ & $6010(30)$ & 58 \\
$\mathrm{H}(14)$ & 3091 & 2502 & 8081 & 39 \\
$\mathrm{H}(19)$ & -8 & 3435 & 7180 & 44 \\
$\mathrm{H}(20)$ & -3385 & 4422 & 6174 & 51 \\
$\mathrm{H}(21)$ & -5554 & 3888 & 5269 & 50 \\
$\mathrm{H}(22)$ & -4192 & 2370 & 5347 & 41 \\
$\mathrm{H}(23)$ & 5428 & -554 & 8180 & 42 \\
$\mathrm{H}(24)$ & 7623 & -94 & 9143 & 45 \\
& & & & \\
\hline
\end{tabular}


Table S10. Torsion angles $\left[{ }^{\circ}\right]$ for $\mathbf{1 q}$.

\begin{tabular}{|c|c|}
\hline $\mathrm{C}(12)-\mathrm{C}(1)-\mathrm{C}(2)-\mathrm{C}(3)$ & $-0.8(6)$ \\
\hline $\mathrm{Br}(1)-\mathrm{C}(1)-\mathrm{C}(2)-\mathrm{C}(3)$ & $178.5(3)$ \\
\hline $\mathrm{C}(1)-\mathrm{C}(2)-\mathrm{C}(3)-\mathrm{C}(4)$ & $1.0(5)$ \\
\hline C(1)-C(2)-C(3)-C(6) & $-178.1(3)$ \\
\hline $\mathrm{B}(1)-\mathrm{O}(1)-\mathrm{C}(4)-\mathrm{C}(11)$ & $-178.2(4)$ \\
\hline $\mathrm{B}(1)-\mathrm{O}(1)-\mathrm{C}(4)-\mathrm{C}(3)$ & $2.4(5)$ \\
\hline $\mathrm{C}(2)-\mathrm{C}(3)-\mathrm{C}(4)-\mathrm{C}(11)$ & $-0.5(6)$ \\
\hline $\mathrm{C}(6)-\mathrm{C}(3)-\mathrm{C}(4)-\mathrm{C}(11)$ & $178.6(4)$ \\
\hline $\mathrm{C}(2)-\mathrm{C}(3)-\mathrm{C}(4)-\mathrm{O}(1)$ & $178.9(3)$ \\
\hline $\mathrm{C}(6)-\mathrm{C}(3)-\mathrm{C}(4)-\mathrm{O}(1)$ & $-1.9(5)$ \\
\hline $\mathrm{C}(10)-\mathrm{C}(5)-\mathrm{C}(6)-\mathrm{C}(7)$ & $0.6(5)$ \\
\hline $\mathrm{B}(1)-\mathrm{C}(5)-\mathrm{C}(6)-\mathrm{C}(7)$ & $177.5(3)$ \\
\hline$C(10)-C(5)-C(6)-C(3)$ & $-178.6(3)$ \\
\hline $\mathrm{B}(1)-\mathrm{C}(5)-\mathrm{C}(6)-\mathrm{C}(3)$ & $-1.7(5)$ \\
\hline $\mathrm{C}(2)-\mathrm{C}(3)-\mathrm{C}(6)-\mathrm{C}(5)$ & $-179.2(3)$ \\
\hline $\mathrm{C}(4)-\mathrm{C}(3)-\mathrm{C}(6)-\mathrm{C}(5)$ & $1.7(5)$ \\
\hline $\mathrm{C}(2)-\mathrm{C}(3)-\mathrm{C}(6)-\mathrm{C}(7)$ & $1.6(5)$ \\
\hline $\mathrm{C}(4)-\mathrm{C}(3)-\mathrm{C}(6)-\mathrm{C}(7)$ & $-177.5(3)$ \\
\hline $\mathrm{C}(5)-\mathrm{C}(6)-\mathrm{C}(7)-\mathrm{C}(8)$ & $-0.9(5)$ \\
\hline $\mathrm{C}(3)-\mathrm{C}(6)-\mathrm{C}(7)-\mathrm{C}(8)$ & 178.2(3) \\
\hline $\mathrm{C}(6)-\mathrm{C}(7)-\mathrm{C}(8)-\mathrm{C}(9)$ & $0.7(6)$ \\
\hline$C(7)-C(8)-C(9)-C(10)$ & $-0.2(6)$ \\
\hline $\mathrm{C}(8)-\mathrm{C}(9)-\mathrm{C}(10)-\mathrm{C}(5)$ & $-0.2(6)$ \\
\hline $\mathrm{C}(6)-\mathrm{C}(5)-\mathrm{C}(10)-\mathrm{C}(9)$ & $-0.1(6)$ \\
\hline $\mathrm{B}(1)-\mathrm{C}(5)-\mathrm{C}(10)-\mathrm{C}(9)$ & $-176.7(4)$ \\
\hline $\mathrm{O}(1)-\mathrm{C}(4)-\mathrm{C}(11)-\mathrm{C}(12)$ & $-179.7(3)$ \\
\hline$C(3)-C(4)-C(11)-C(12)$ & $-0.2(6)$ \\
\hline $\mathrm{C}(2)-\mathrm{C}(1)-\mathrm{C}(12)-\mathrm{C}(11)$ & $0.0(6)$ \\
\hline $\mathrm{Br}(1)-\mathrm{C}(1)-\mathrm{C}(12)-\mathrm{C}(11)$ & $-179.3(3)$ \\
\hline $\mathrm{C}(4)-\mathrm{C}(11)-\mathrm{C}(12)-\mathrm{C}(1)$ & $0.5(6)$ \\
\hline $\mathrm{C}(4)-\mathrm{O}(1)-\mathrm{B}(1)-\mathrm{O}(2)$ & $177.6(3)$ \\
\hline $\mathrm{C}(4)-\mathrm{O}(1)-\mathrm{B}(1)-\mathrm{C}(5)$ & $-2.4(5)$ \\
\hline $\mathrm{C}(10)-\mathrm{C}(5)-\mathrm{B}(1)-\mathrm{O}(2)$ & $-1.2(6)$ \\
\hline $\mathrm{C}(6)-\mathrm{C}(5)-\mathrm{B}(1)-\mathrm{O}(2)$ & $-177.9(4)$ \\
\hline $\mathrm{C}(10)-\mathrm{C}(5)-\mathrm{B}(1)-\mathrm{O}(1)$ & $178.8(4)$ \\
\hline $\mathrm{C}(6)-\mathrm{C}(5)-\mathrm{B}(1)-\mathrm{O}(1)$ & $2.1(5)$ \\
\hline $\mathrm{C}(24)-\mathrm{C}(13)-\mathrm{C}(14)-\mathrm{C}(15)$ & $0.4(6)$ \\
\hline $\mathrm{Br}(2)-\mathrm{C}(13)-\mathrm{C}(14)-\mathrm{C}(15)$ & $-178.8(3)$ \\
\hline $\mathrm{C}(13)-\mathrm{C}(14)-\mathrm{C}(15)-\mathrm{C}(16)$ & $-0.5(6)$ \\
\hline $\mathrm{C}(13)-\mathrm{C}(14)-\mathrm{C}(15)-\mathrm{C}(18)$ & $179.8(3)$ \\
\hline $\mathrm{B}(2)-\mathrm{O}(3)-\mathrm{C}(16)-\mathrm{C}(23)$ & $179.9(3)$ \\
\hline $\mathrm{B}(2)-\mathrm{O}(3)-\mathrm{C}(16)-\mathrm{C}(15)$ & $-0.8(5)$ \\
\hline $\mathrm{C}(14)-\mathrm{C}(15)-\mathrm{C}(16)-\mathrm{O}(3)$ & $-179.1(3)$ \\
\hline $\mathrm{C}(18)-\mathrm{C}(15)-\mathrm{C}(16)-\mathrm{O}(3)$ & $0.6(6)$ \\
\hline $\mathrm{C}(14)-\mathrm{C}(15)-\mathrm{C}(16)-\mathrm{C}(23)$ & $0.2(6)$ \\
\hline
\end{tabular}




$\begin{array}{lc}\mathrm{C}(18)-\mathrm{C}(15)-\mathrm{C}(16)-\mathrm{C}(23) & 179.9(3) \\ \mathrm{C}(22)-\mathrm{C}(17)-\mathrm{C}(18)-\mathrm{C}(19) & 0.0(6) \\ \mathrm{B}(2)-\mathrm{C}(17)-\mathrm{C}(18)-\mathrm{C}(19) & 179.8(3) \\ \mathrm{C}(22)-\mathrm{C}(17)-\mathrm{C}(18)-\mathrm{C}(15) & 178.7(3) \\ \mathrm{B}(2)-\mathrm{C}(17)-\mathrm{C}(18)-\mathrm{C}(15) & -1.5(5) \\ \mathrm{C}(16)-\mathrm{C}(15)-\mathrm{C}(18)-\mathrm{C}(19) & 179.2(4) \\ \mathrm{C}(14)-\mathrm{C}(15)-\mathrm{C}(18)-\mathrm{C}(19) & -1.1(6) \\ \mathrm{C}(16)-\mathrm{C}(15)-\mathrm{C}(18)-\mathrm{C}(17) & 0.6(5) \\ \mathrm{C}(14)-\mathrm{C}(15)-\mathrm{C}(18)-\mathrm{C}(17) & -179.7(4) \\ \mathrm{C}(17)-\mathrm{C}(18)-\mathrm{C}(19)-\mathrm{C}(20) & -0.5(6) \\ \mathrm{C}(15)-\mathrm{C}(18)-\mathrm{C}(19)-\mathrm{C}(20) & -179.1(4) \\ \mathrm{C}(18)-\mathrm{C}(19)-\mathrm{C}(20)-\mathrm{C}(21) & 1.0(6) \\ \mathrm{C}(19)-\mathrm{C}(20)-\mathrm{C}(21)-\mathrm{C}(22) & -1.1(7) \\ \mathrm{C}(20)-\mathrm{C}(21)-\mathrm{C}(22)-\mathrm{C}(17) & 0.6(6) \\ \mathrm{C}(18)-\mathrm{C}(17)-\mathrm{C}(22)-\mathrm{C}(21) & -0.1(6) \\ \mathrm{B}(2)-\mathrm{C}(17)-\mathrm{C}(22)-\mathrm{C}(21) & -179.9(4) \\ \mathrm{O}(3)-\mathrm{C}(16)-\mathrm{C}(23)-\mathrm{C}(24) & 179.5(4) \\ \mathrm{C}(15)-\mathrm{C}(16)-\mathrm{C}(23)-\mathrm{C}(24) & 0.2(6) \\ \mathrm{C}(14)-\mathrm{C}(13)-\mathrm{C}(24)-\mathrm{C}(23) & 0.0(6) \\ \mathrm{Br}(2)-\mathrm{C}(13)-\mathrm{C}(24)-\mathrm{C}(23) & 179.2(3) \\ \mathrm{C}(16)-\mathrm{C}(23)-\mathrm{C}(24)-\mathrm{C}(13) & -0.3(6) \\ \mathrm{C}(16)-\mathrm{O}(3)-\mathrm{B}(2)-\mathrm{O}(4) & 179.5(3) \\ \mathrm{C}(16)-\mathrm{O}(3)-\mathrm{B}(2)-\mathrm{C}(17) & -0.3(5) \\ \mathrm{C}(22)-\mathrm{C}(17)-\mathrm{B}(2)-\mathrm{O}(4) & 1.4(6) \\ \mathrm{C}(18)-\mathrm{C}(17)-\mathrm{B}(2)-\mathrm{O}(4) & -178.3(4) \\ \mathrm{C}(22)-\mathrm{C}(17)-\mathrm{B}(2)-\mathrm{O}(3) & -178.8(3) \\ \mathrm{C}(18)-\mathrm{C}(17)-\mathrm{B}(2)-\mathrm{O}(3) & 1.4(6) \\ & \end{array}$

Symmetry transformations used to generate equivalent atoms: 


\section{${ }^{1} \mathrm{H},{ }^{13} \mathrm{C}$, and ${ }^{19} \mathrm{~F}$ NMR Spectra of Compounds}

${ }^{1} \mathrm{H}$ NMR $\left(\mathrm{CDCl}_{3}\right.$ and 1 drop of $\left.\mathrm{CD}_{3} \mathrm{OD}, 400 \mathrm{MHz}\right)$ and ${ }^{13} \mathrm{C} \mathrm{NMR}\left(\mathrm{CDCl}_{3}, 100 \mathrm{MHz}\right)$ spectra of 11
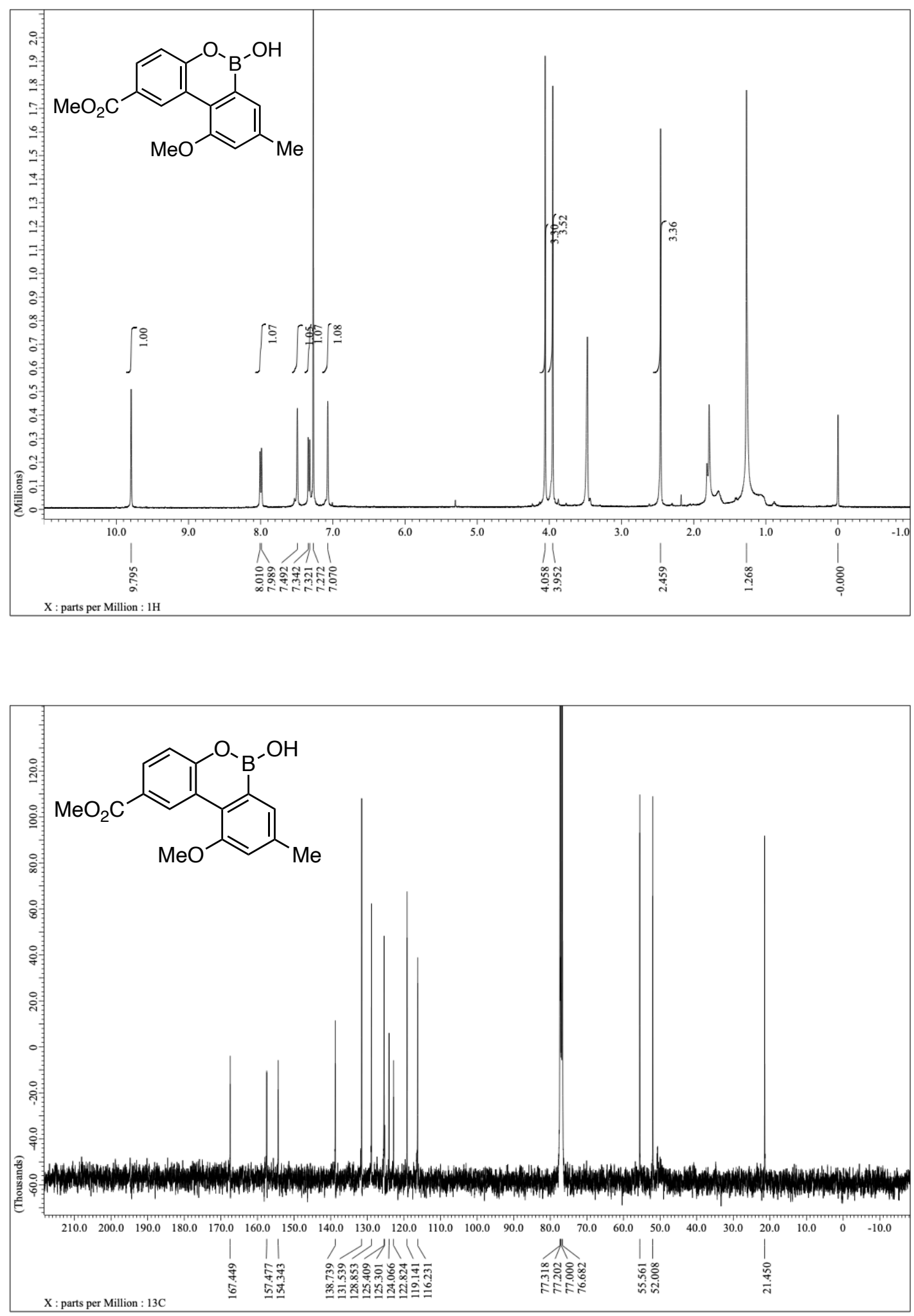
${ }^{1} \mathrm{H}$ NMR (400 MHz) and ${ }^{13} \mathrm{C}$ NMR $(100 \mathrm{MHz})$ spectra of $\mathbf{1 m}\left(\mathrm{CDCl}_{3}\right)$
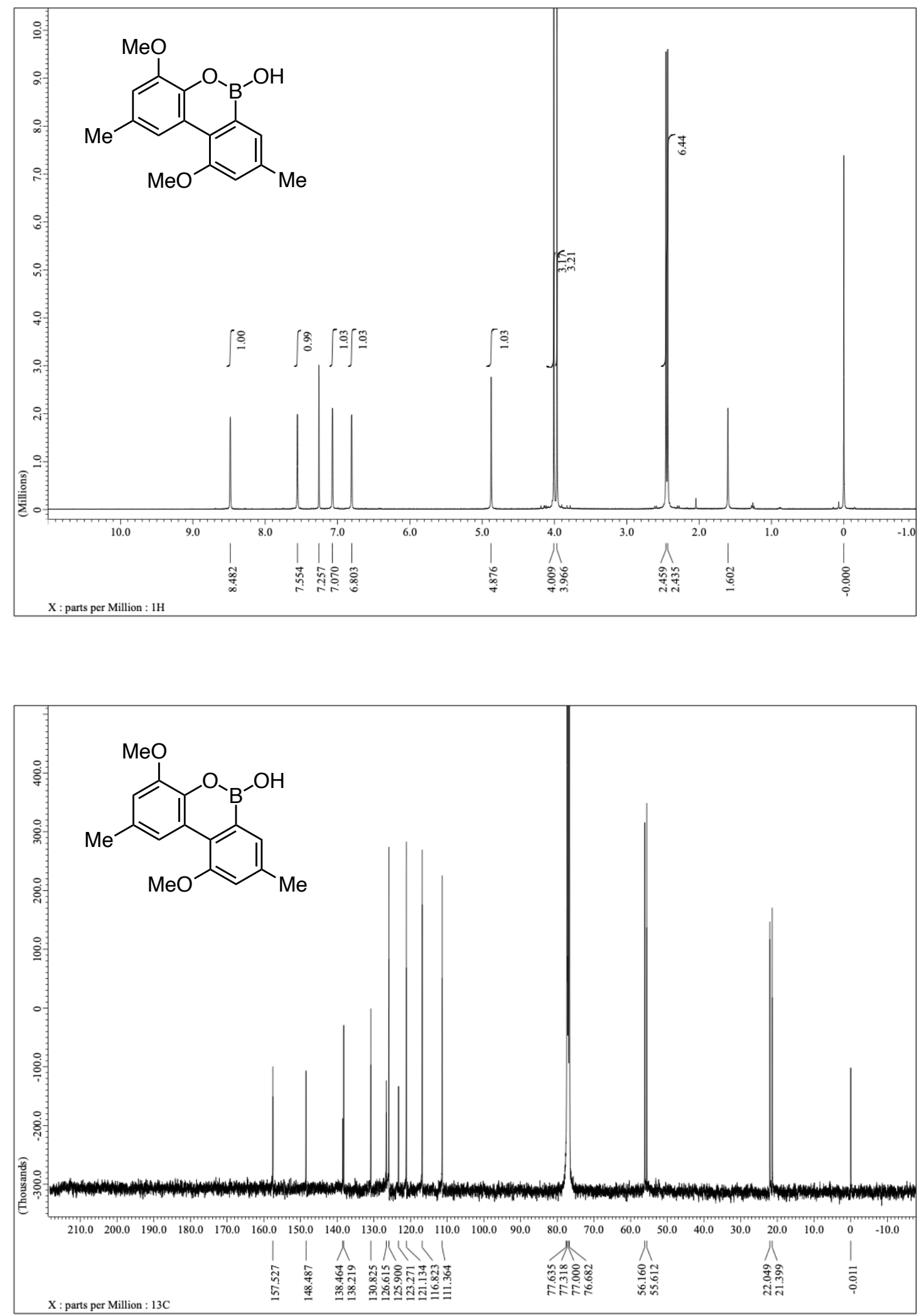
${ }^{1} \mathrm{H}$ NMR $(400 \mathrm{MHz})$ and ${ }^{13} \mathrm{C}$ NMR $(100 \mathrm{MHz})$ spectra of $\mathbf{2 a}\left(\mathrm{CDCl}_{3}\right)$
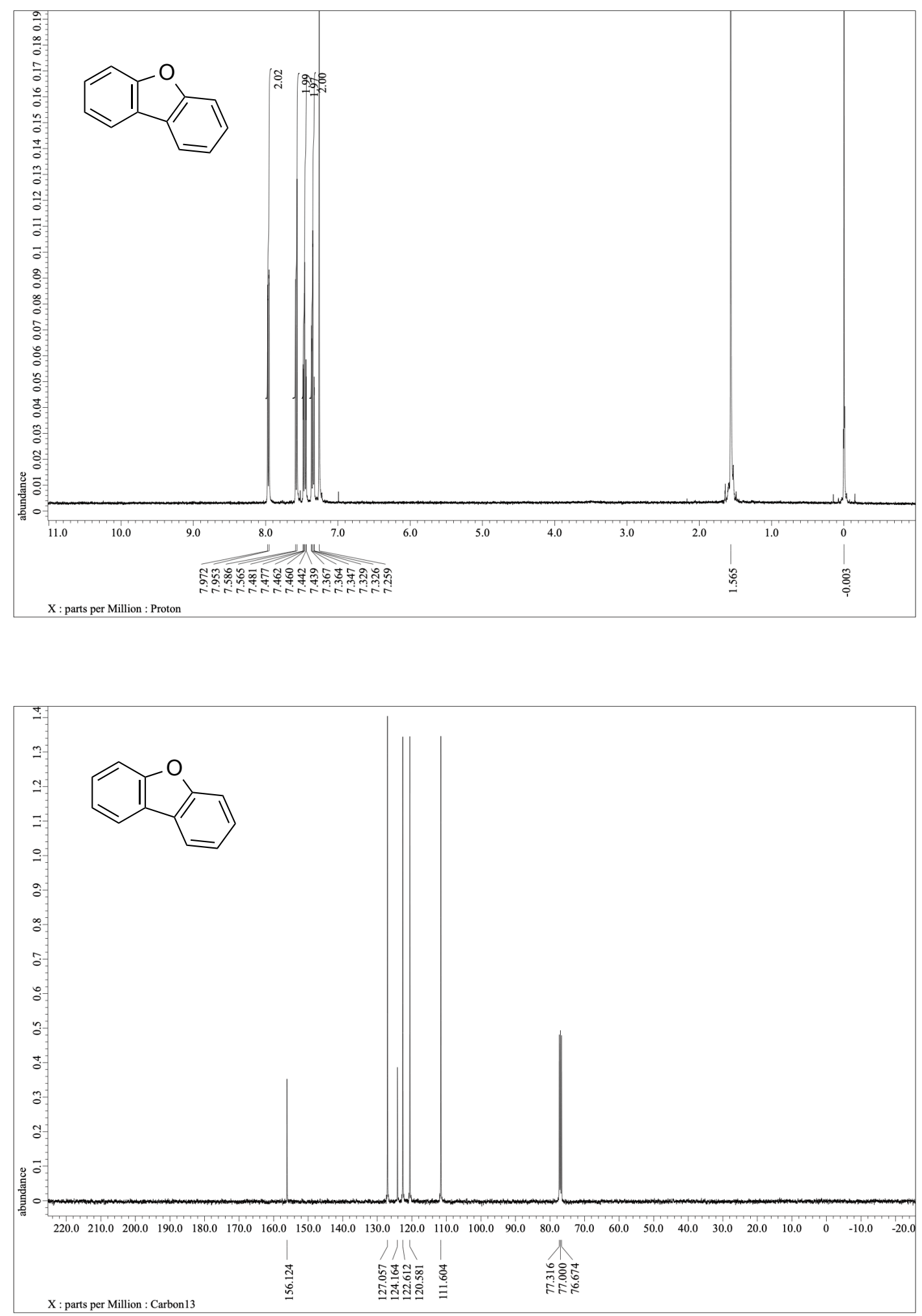
${ }^{1} \mathrm{H}$ NMR $(400 \mathrm{MHz})$ and ${ }^{13} \mathrm{C}$ NMR $(100 \mathrm{MHz})$ spectra of $\mathbf{2 b}(=\mathbf{2 h})\left(\mathrm{CDCl}_{3}\right)$
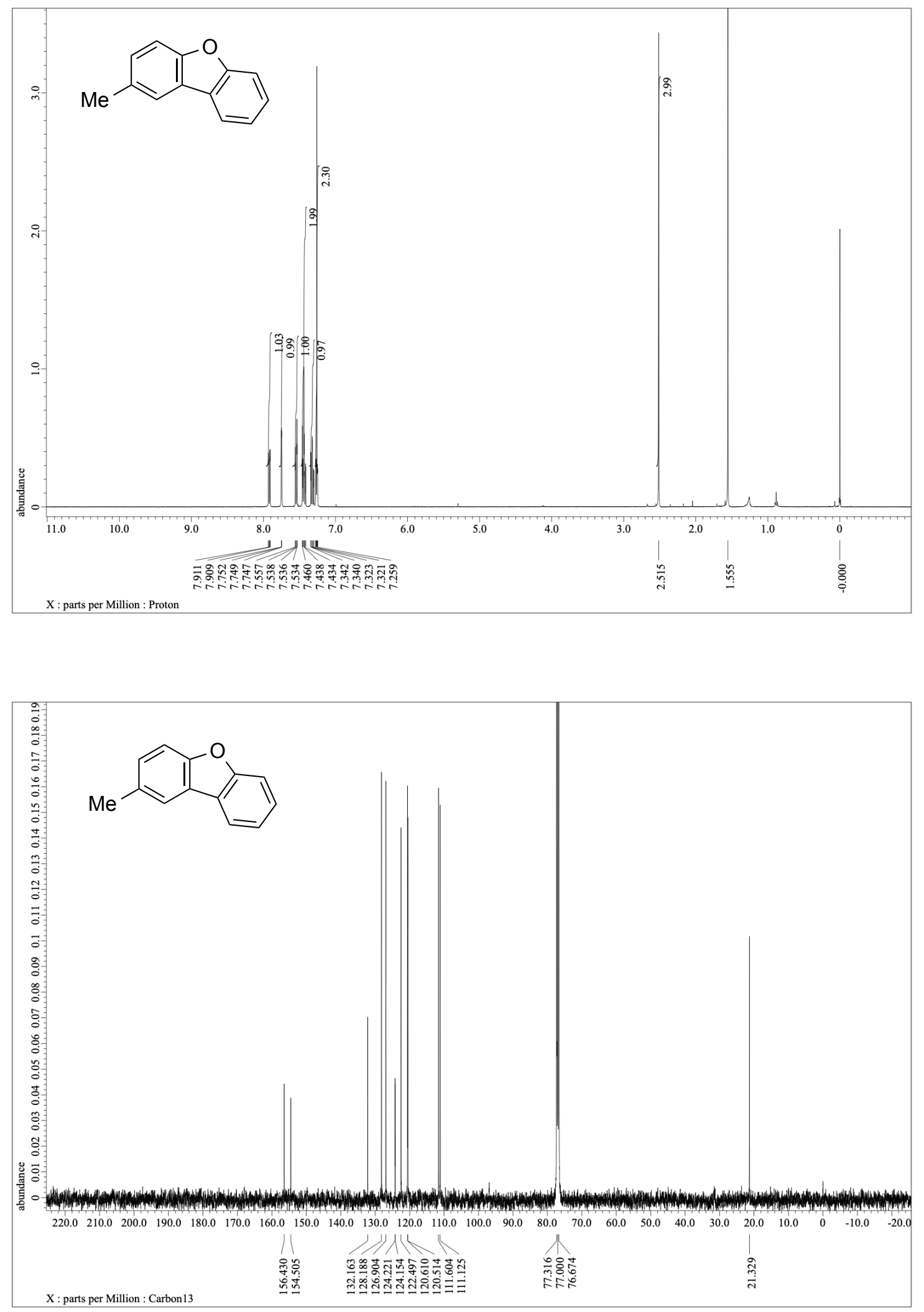
${ }^{1} \mathrm{H}$ NMR $(400 \mathrm{MHz})$ and ${ }^{13} \mathrm{C}$ NMR $(100 \mathrm{MHz})$ spectra of $2 \mathbf{c}\left(\mathrm{CDCl}_{3}\right)$
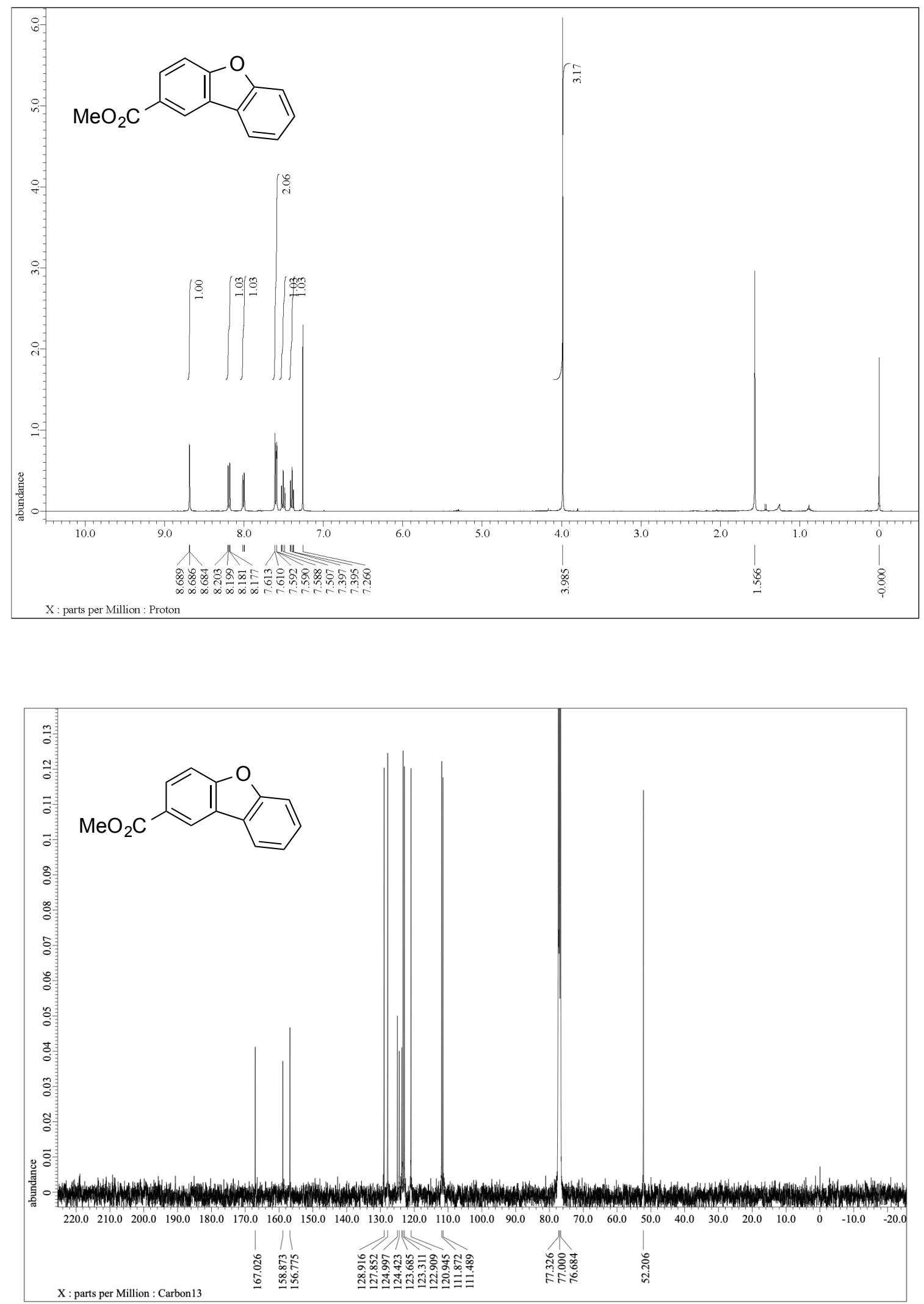
${ }^{1} \mathrm{H}$ NMR (400 MHz), ${ }^{13} \mathrm{C}$ NMR (100 MHz), and ${ }^{19} \mathrm{~F}$ NMR (372.5 MHz) spectra of $2 \mathbf{d}\left(\mathrm{CDCl}_{3}\right)$
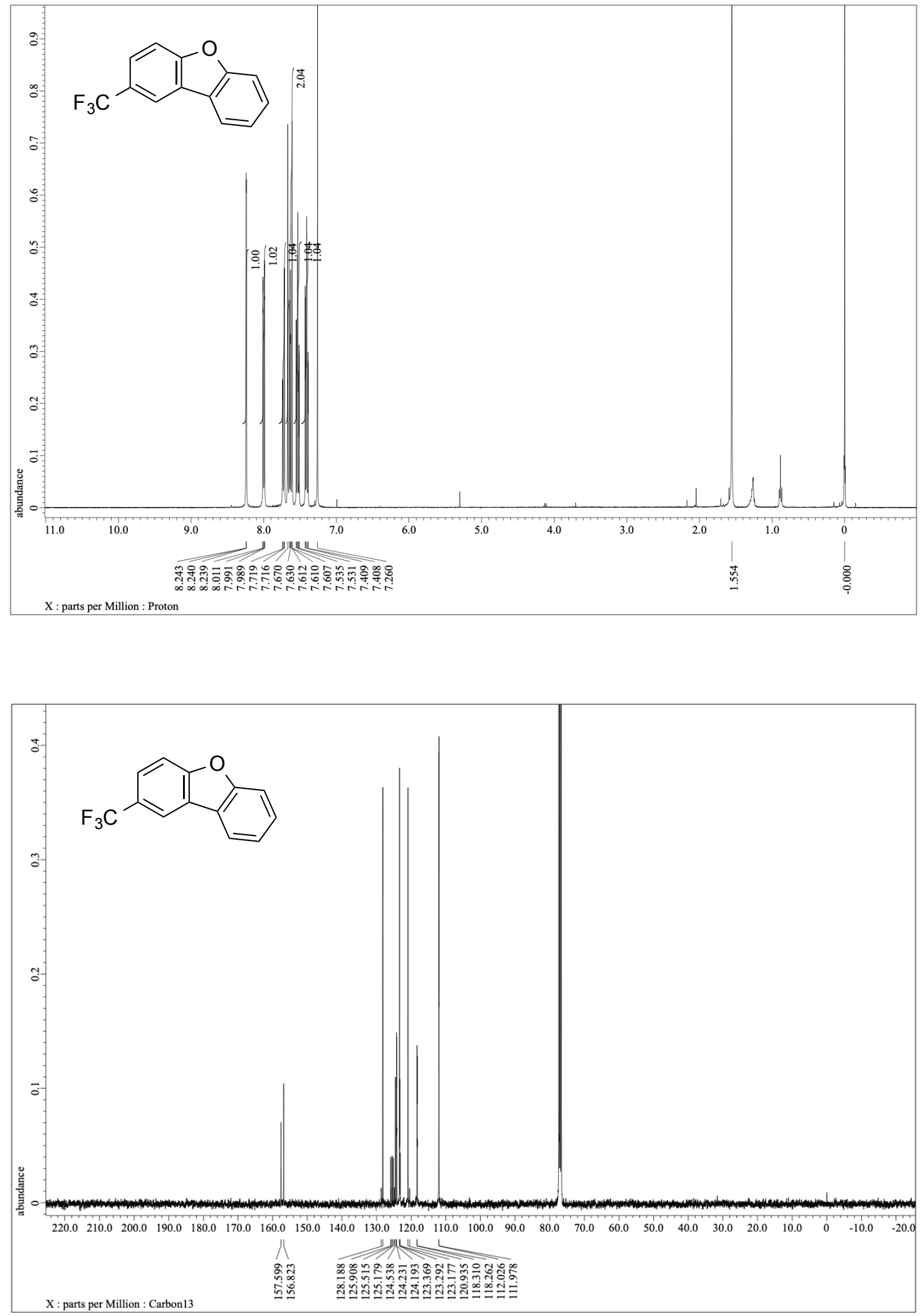


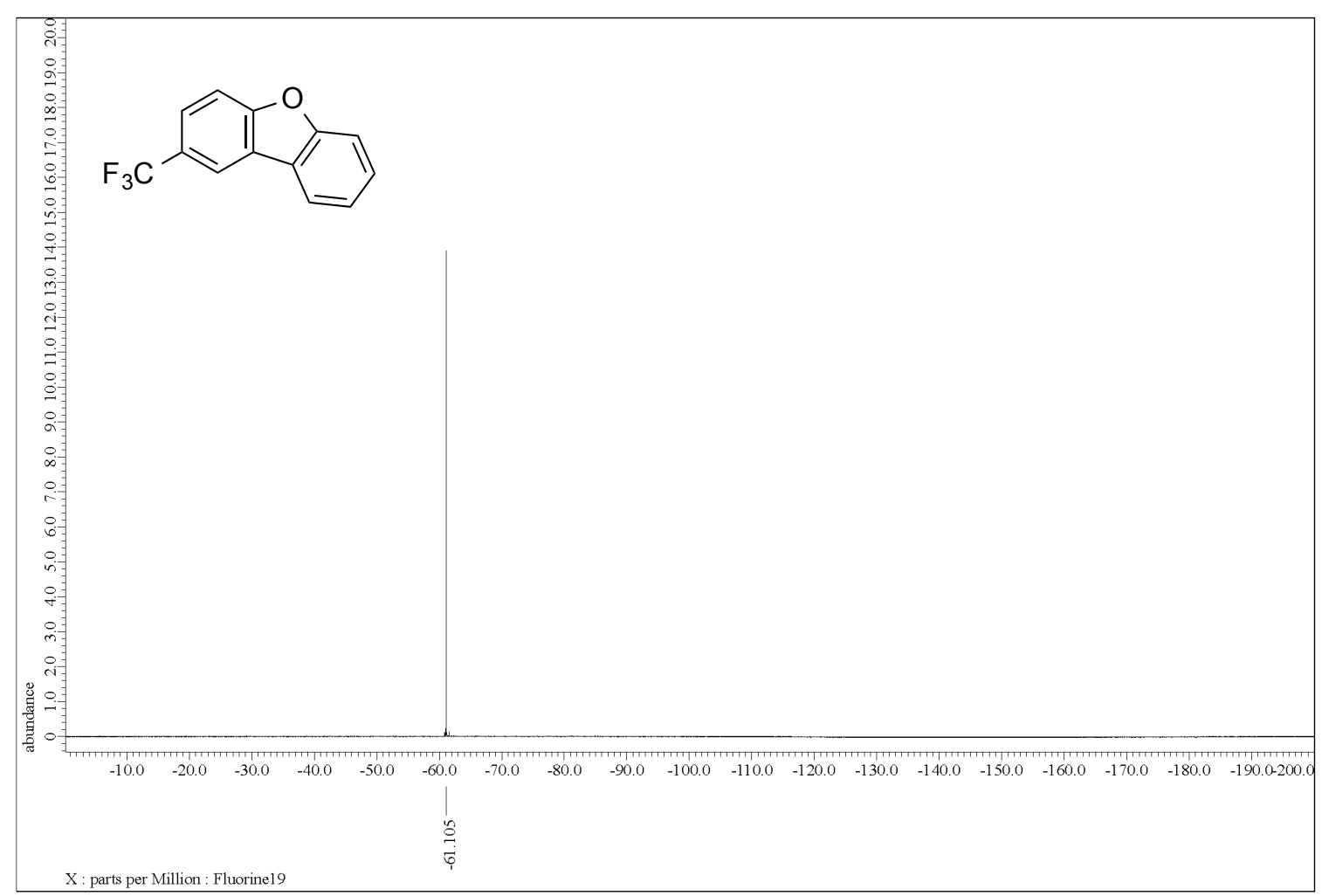


${ }^{1} \mathrm{H}$ NMR $(400 \mathrm{MHz})$ and ${ }^{13} \mathrm{C}$ NMR $(100 \mathrm{MHz})$ spectra of $\mathbf{2 e}\left(\mathrm{CDCl}_{3}\right)$
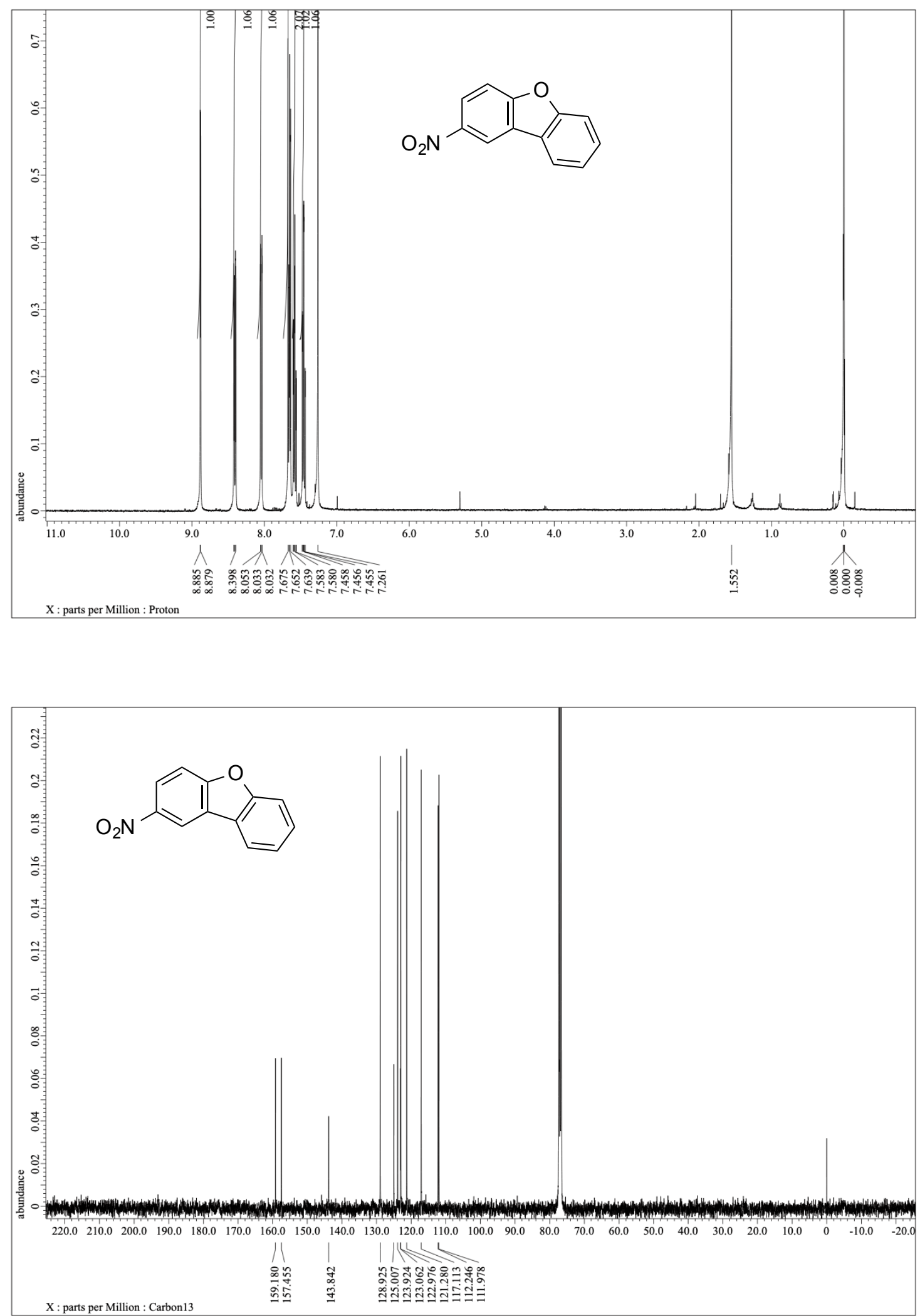
${ }^{1} \mathrm{H}$ NMR (400 MHz), ${ }^{13} \mathrm{C}$ NMR (100 MHz), and ${ }^{19} \mathrm{~F}$ NMR (372.5 MHz) spectra of $2 \mathbf{f}\left(\mathrm{CDCl}_{3}\right)$
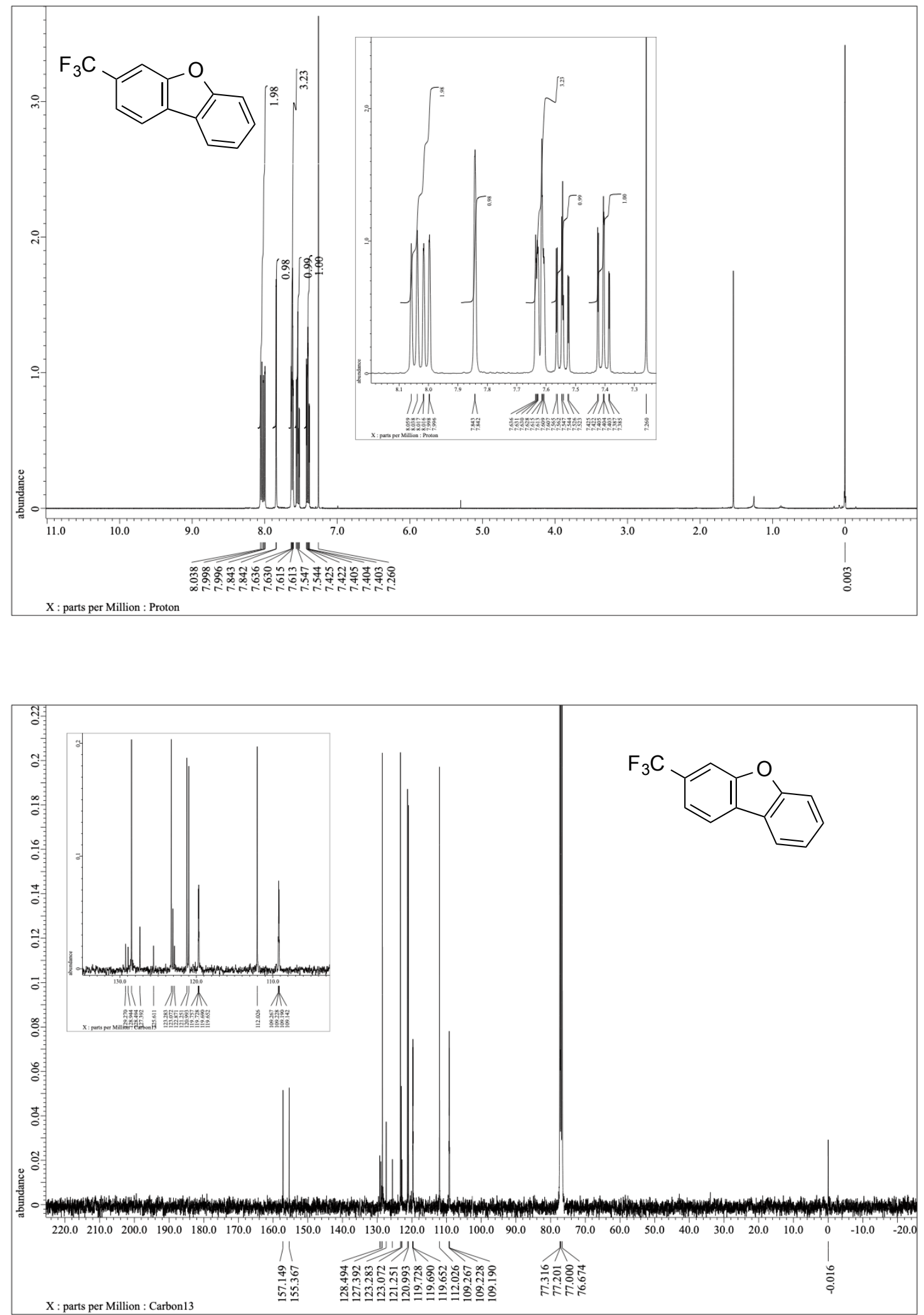


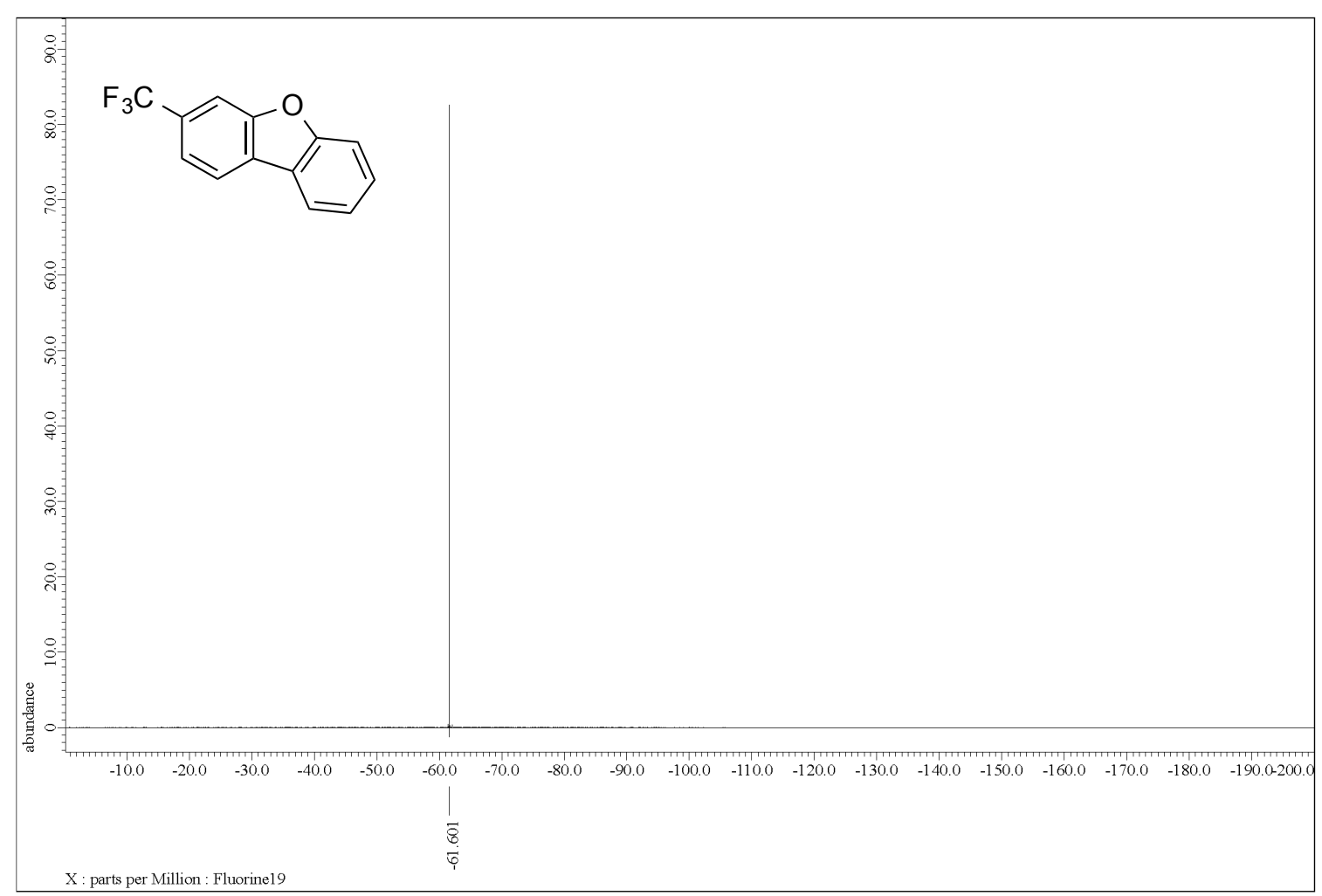


${ }^{1} \mathrm{H}$ NMR $(400 \mathrm{MHz})$ and ${ }^{13} \mathrm{C}$ NMR $(100 \mathrm{MHz})$ spectra of $\mathbf{2 g}\left(\mathrm{CDCl}_{3}\right)$
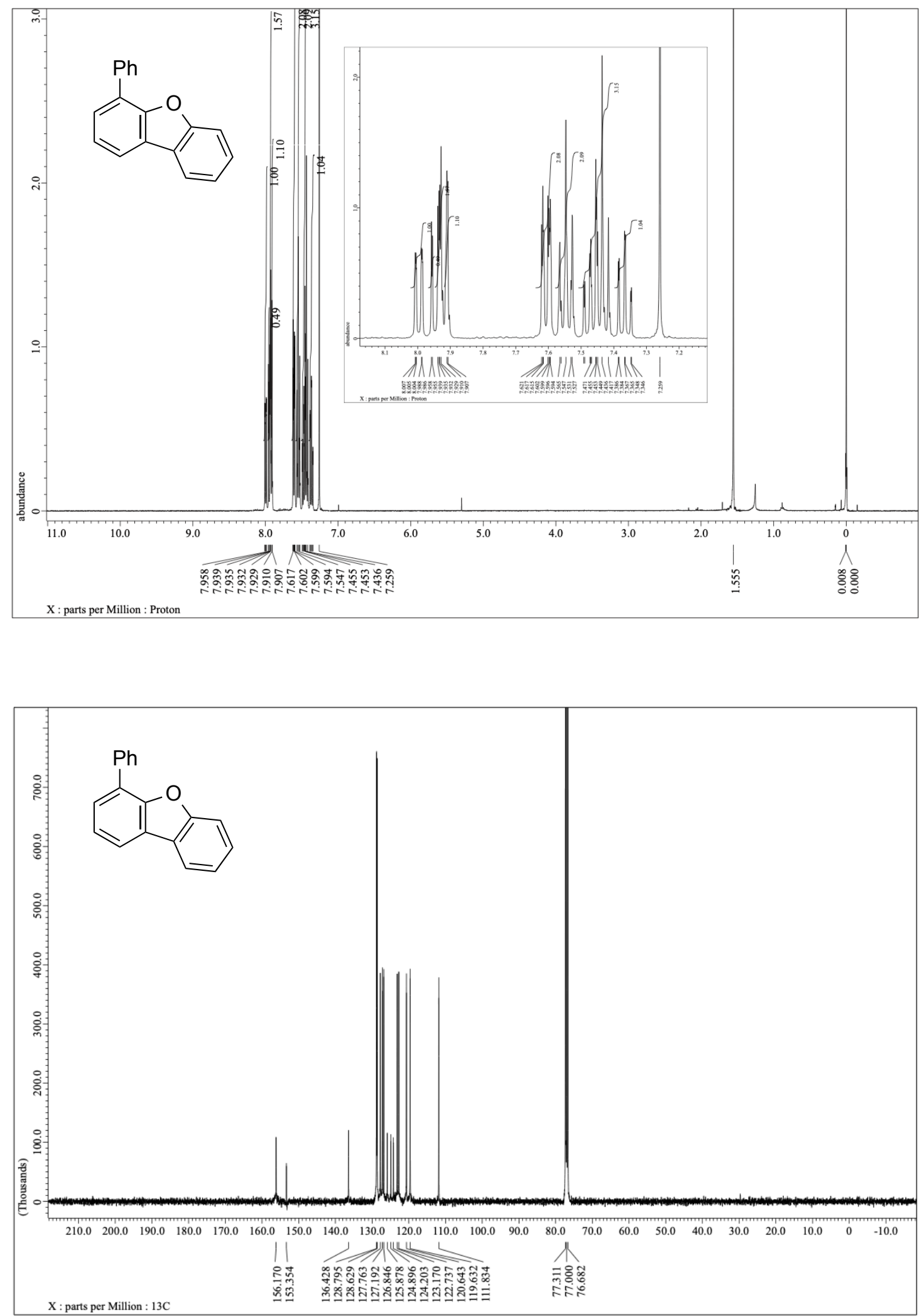
${ }^{1} \mathrm{H}$ NMR $(400 \mathrm{MHz})$ and ${ }^{13} \mathrm{C}$ NMR $(100 \mathrm{MHz})$ spectra of $2 \mathbf{i}\left(\mathrm{CDCl}_{3}\right)$
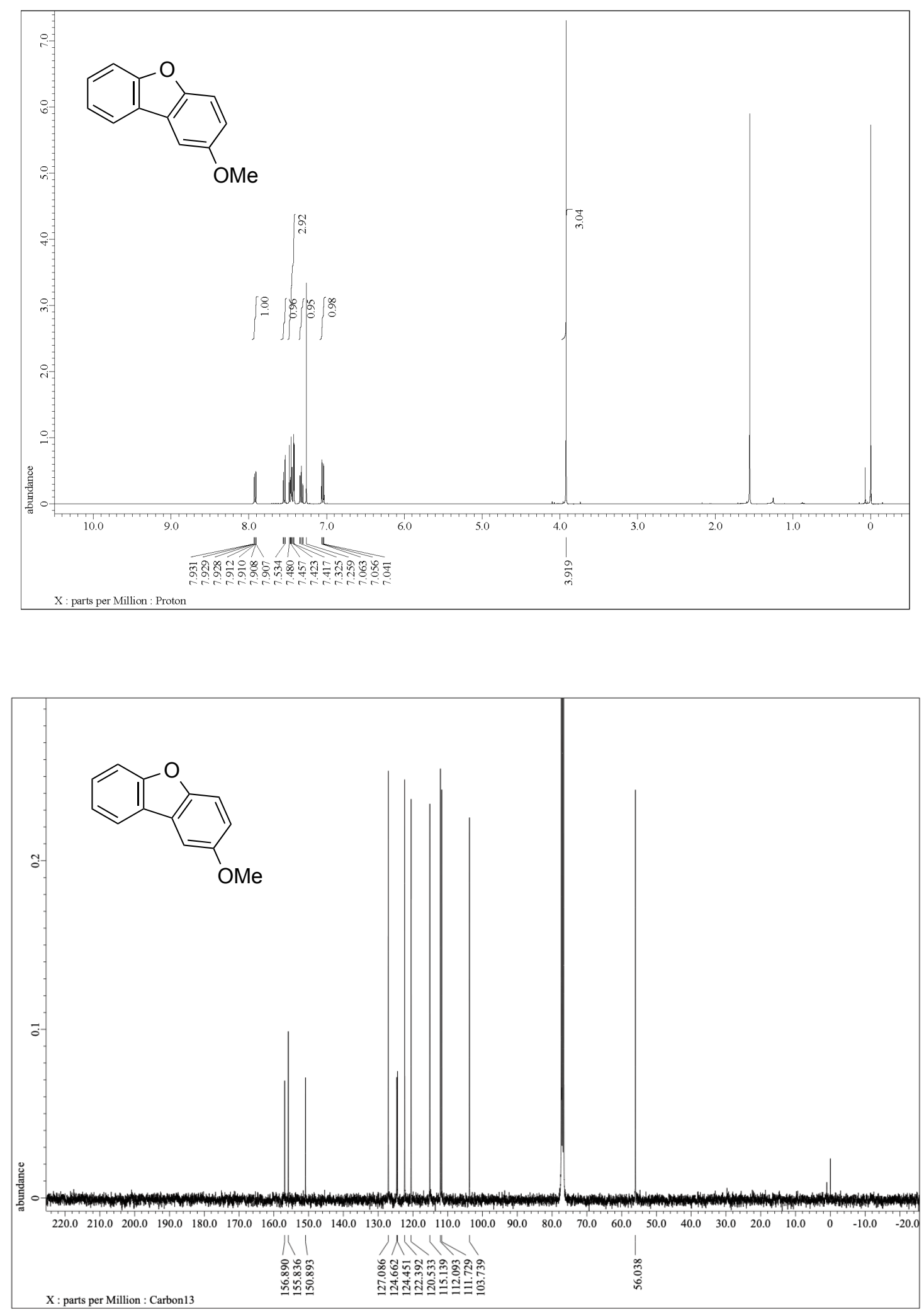
${ }^{1} \mathrm{H}$ NMR (400 MHz), ${ }^{13} \mathrm{C}$ NMR (100 MHz), and ${ }^{19} \mathrm{~F}$ NMR (372.5 MHz) spectra of $\mathbf{2} \mathbf{j}\left(\mathrm{CDCl}_{3}\right)$
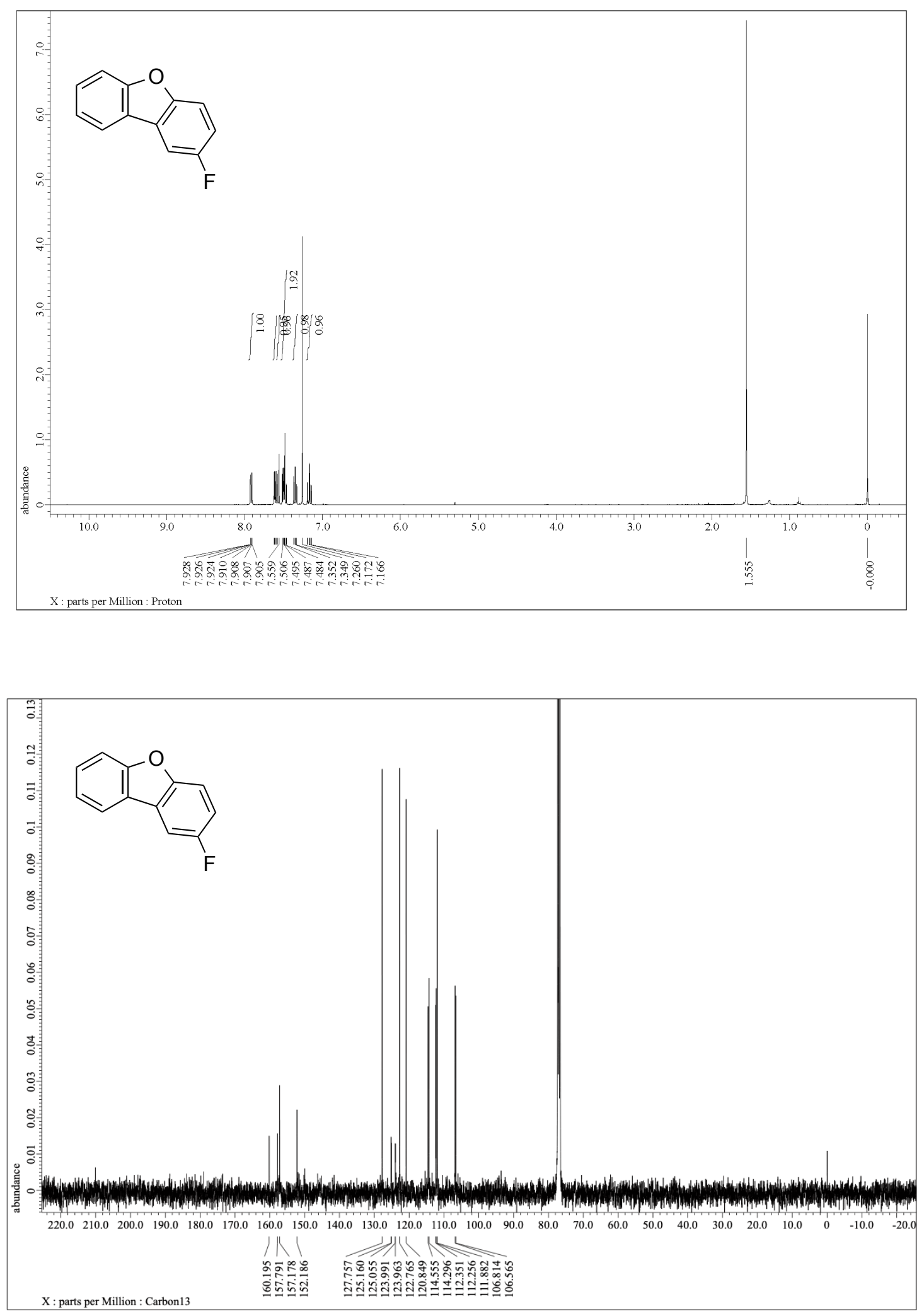

S49 


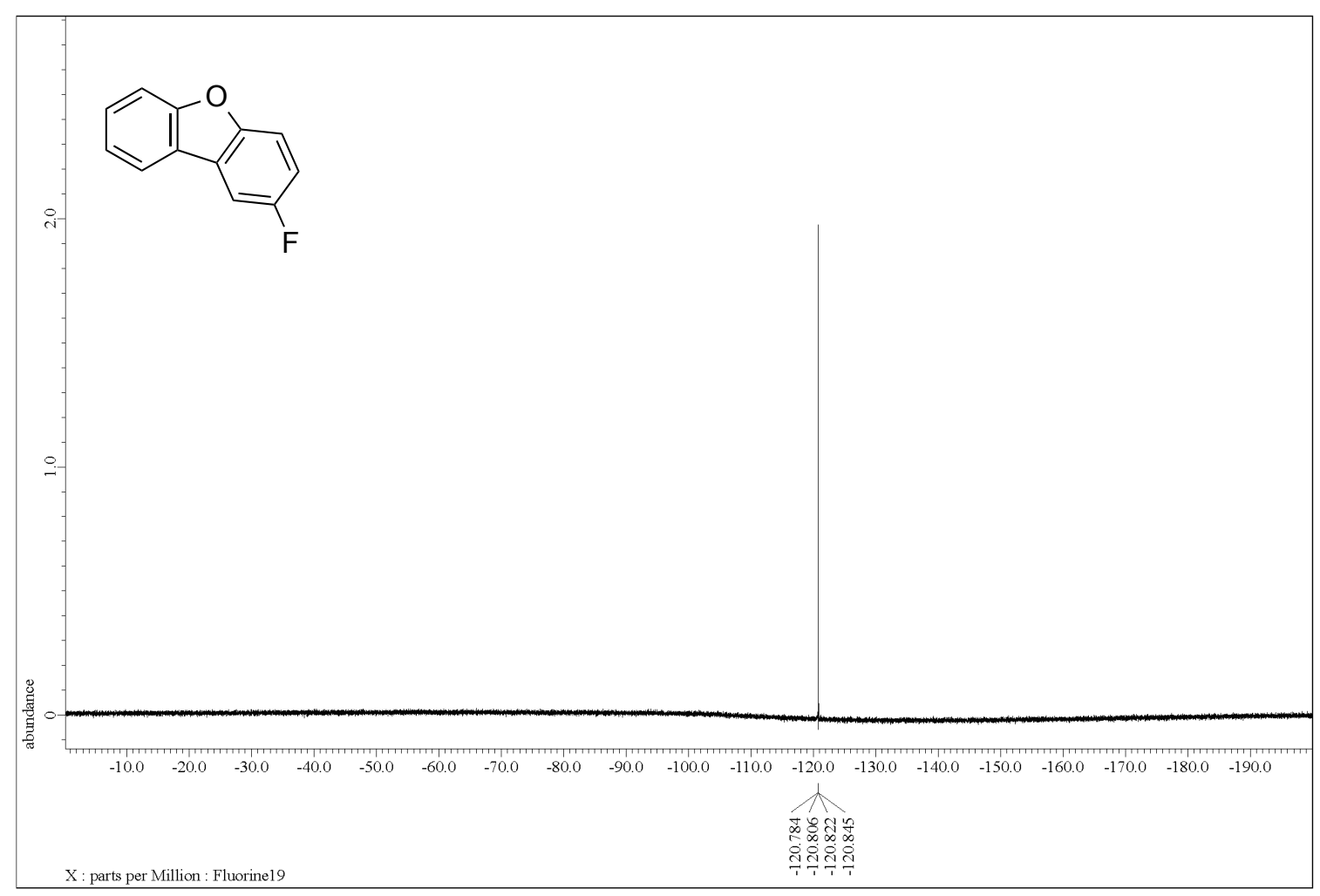


${ }^{1} \mathrm{H}$ NMR (400 MHz), ${ }^{13} \mathrm{C}$ NMR (100 MHz), and ${ }^{19} \mathrm{~F}$ NMR $(372.5 \mathrm{MHz})$ spectra of $2 \mathbf{k}\left(\mathrm{CDCl}_{3}\right)$
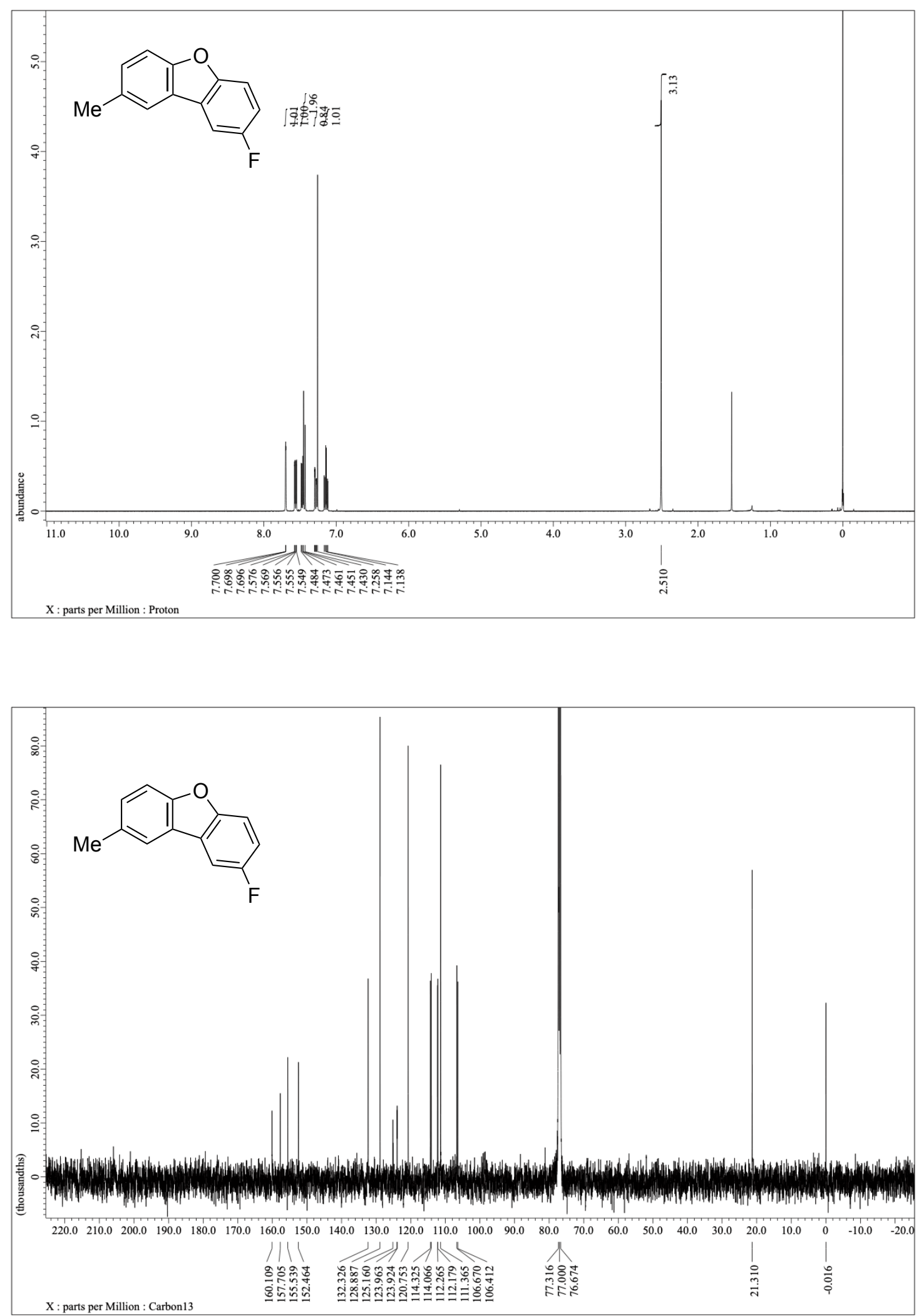


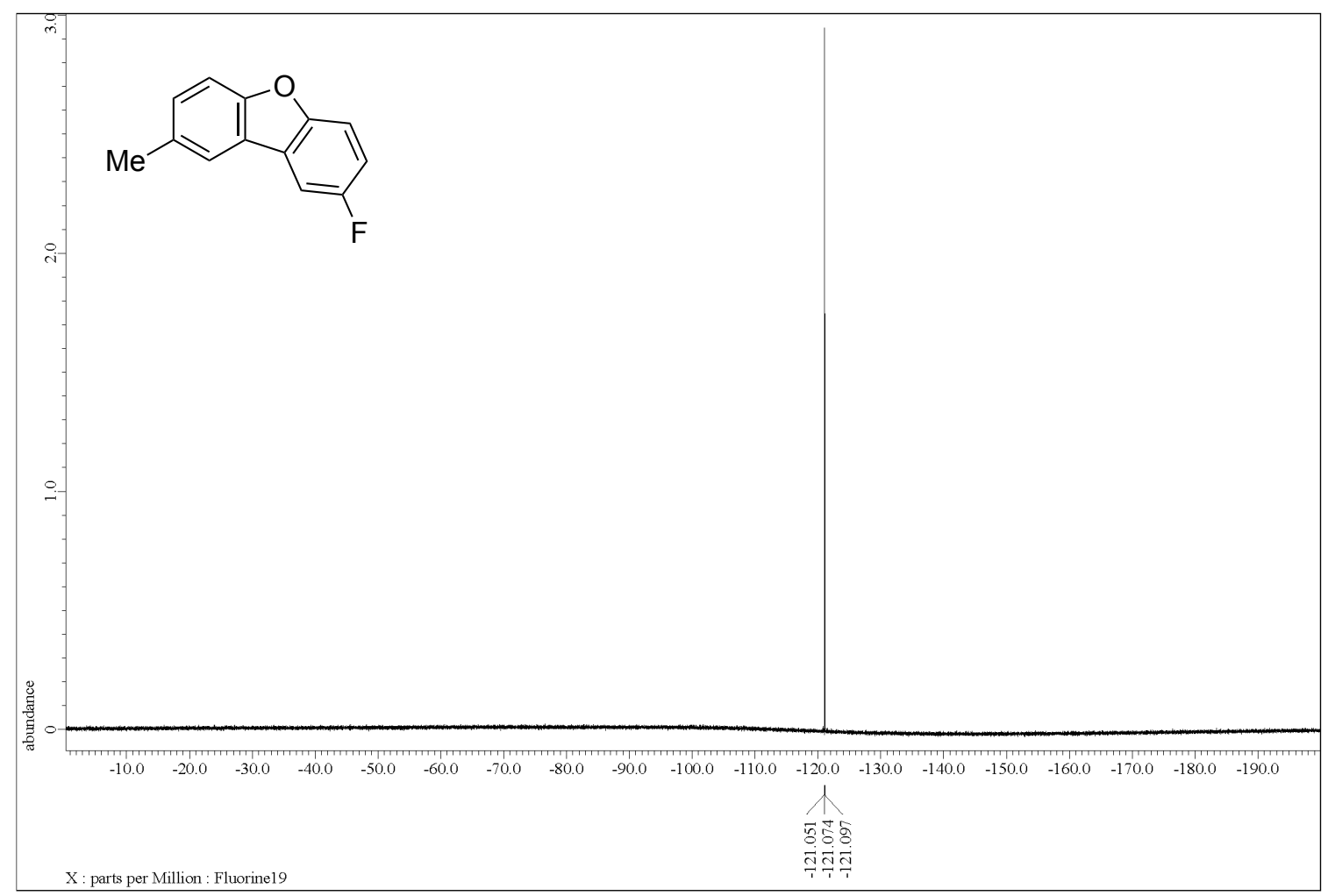


${ }^{1} \mathrm{H}$ NMR $(400 \mathrm{MHz})$ and ${ }^{13} \mathrm{C}$ NMR $(100 \mathrm{MHz})$ spectra of $2 \mathbf{l}\left(\mathrm{CDCl}_{3}\right)$
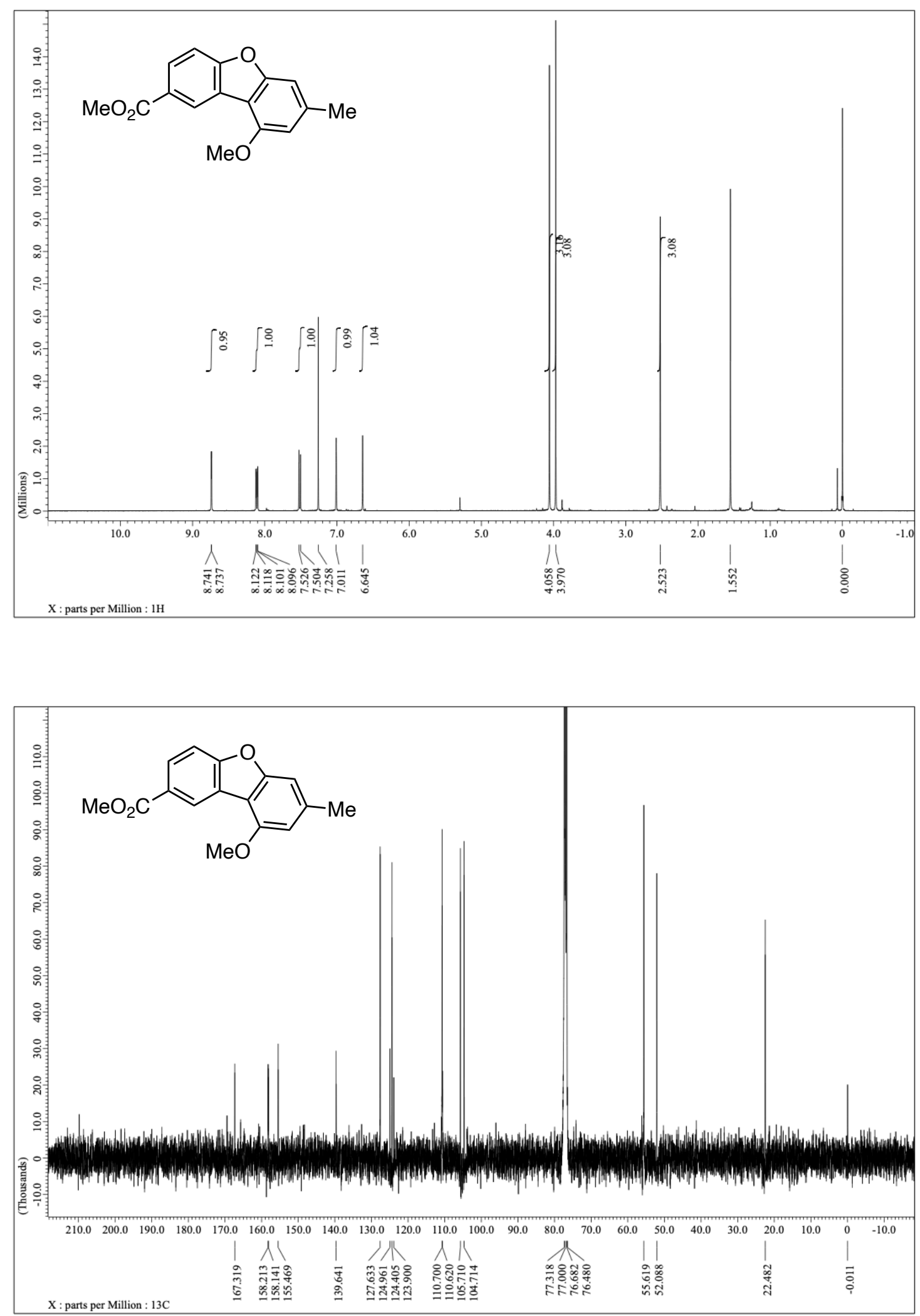
${ }^{1} \mathrm{H}$ NMR $(400 \mathrm{MHz})$ and ${ }^{13} \mathrm{C}$ NMR $(100 \mathrm{MHz})$ spectra of $\mathbf{2 m}\left(\mathrm{CDCl}_{3}\right)$
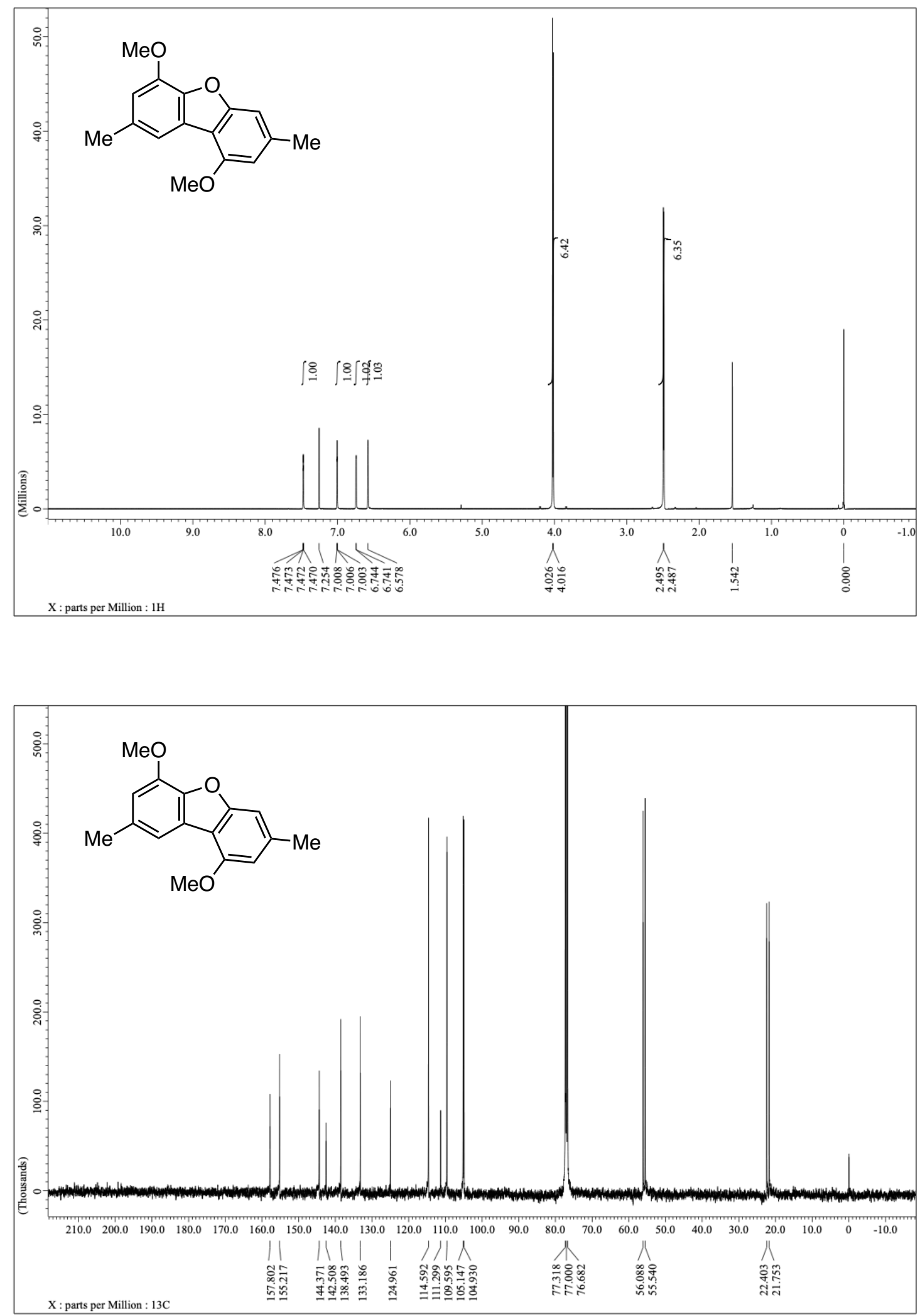
${ }^{1} \mathrm{H}$ NMR $(400 \mathrm{MHz})$ and ${ }^{13} \mathrm{C}$ NMR $(100 \mathrm{MHz})$ spectra of $\mathbf{2 n}\left(\mathrm{CDCl}_{3}\right)$
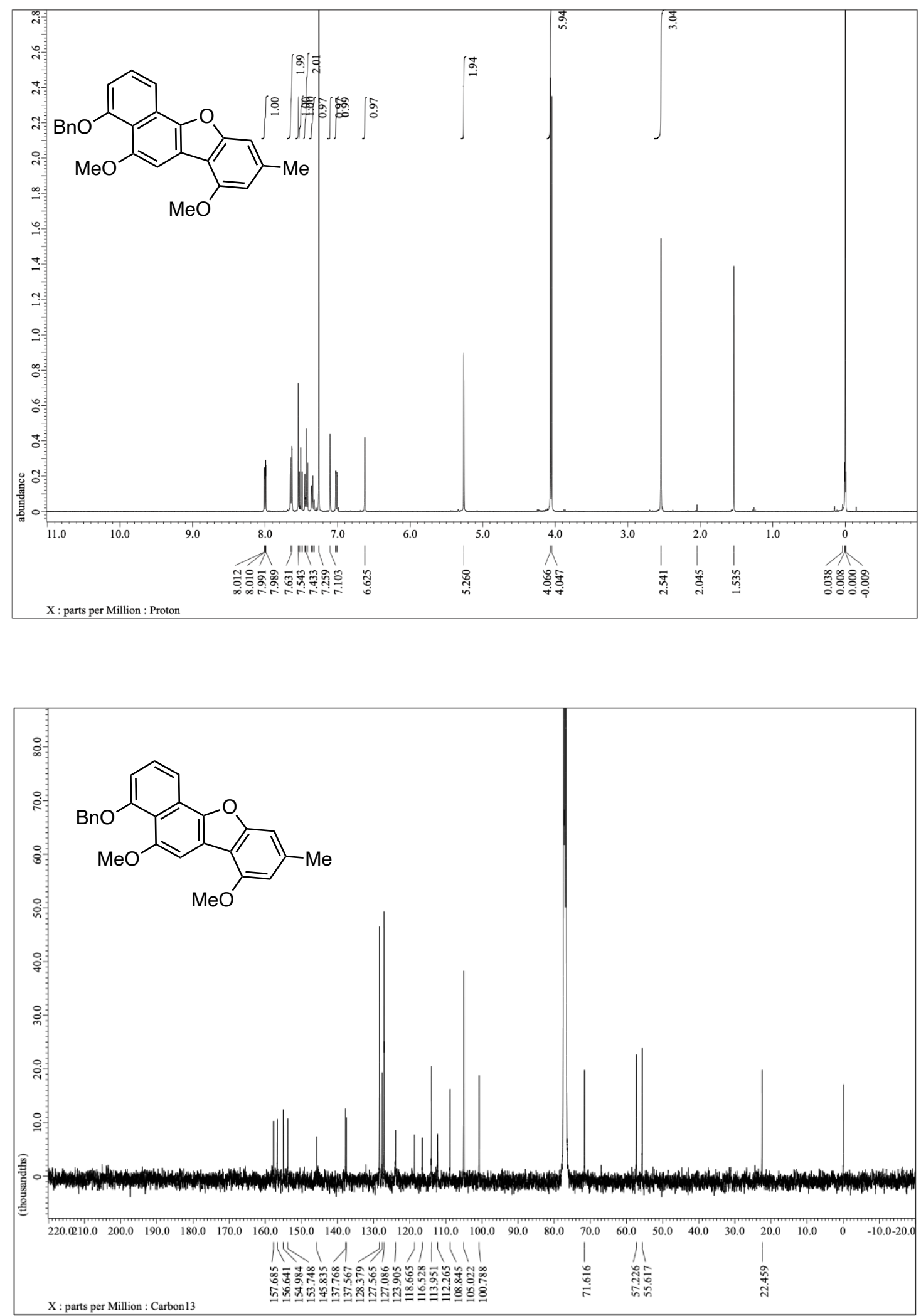
${ }^{1} \mathrm{H}$ NMR $(400 \mathrm{MHz})$ and ${ }^{13} \mathrm{C}$ NMR $(100 \mathrm{MHz})$ spectra of $20\left(\mathrm{CDCl}_{3}\right)$
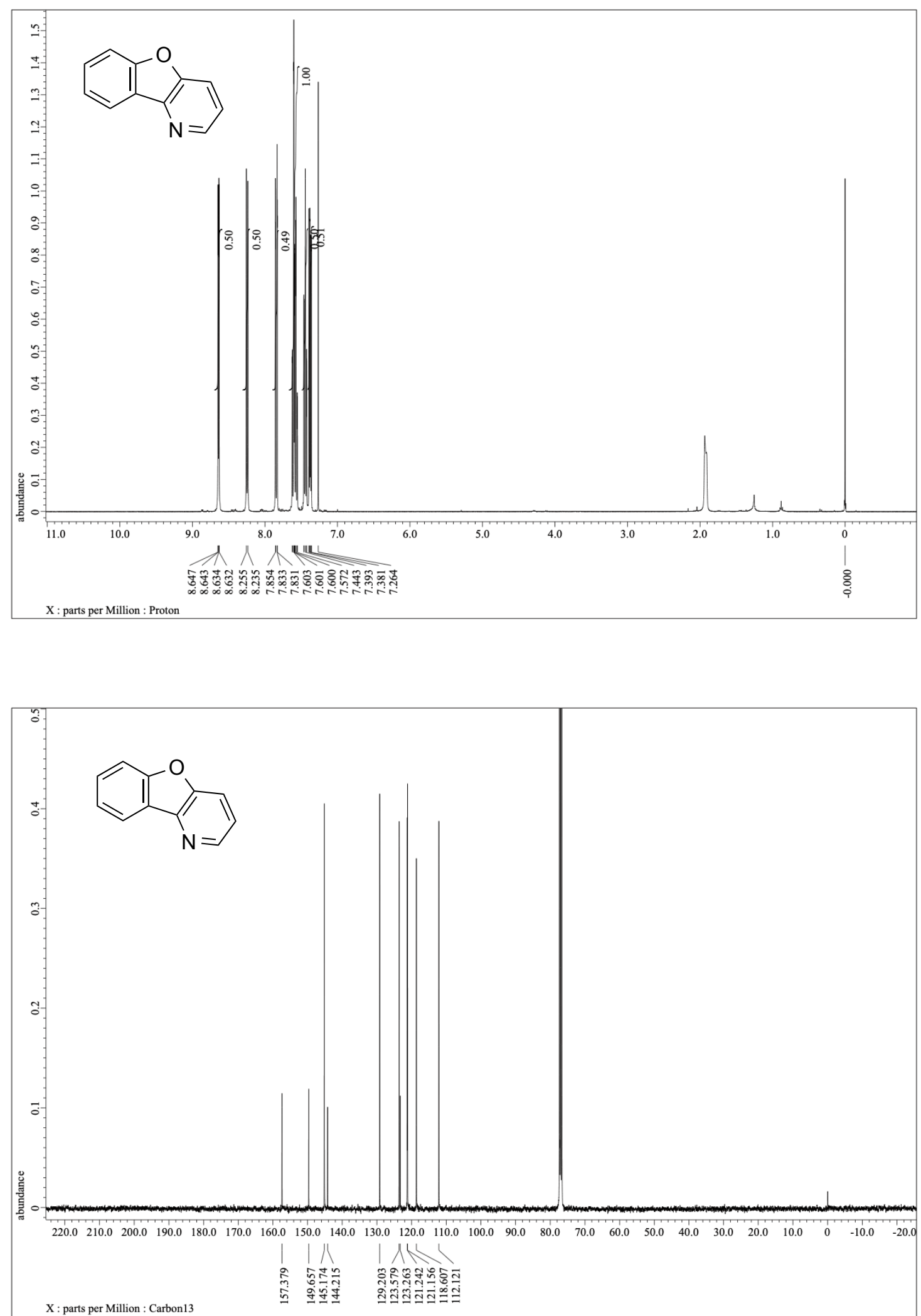
${ }^{1} \mathrm{H}$ NMR $(500 \mathrm{MHz})$ and ${ }^{13} \mathrm{C}$ NMR (126 MHz) spectra of 1q (acetone- $d_{6}$ )
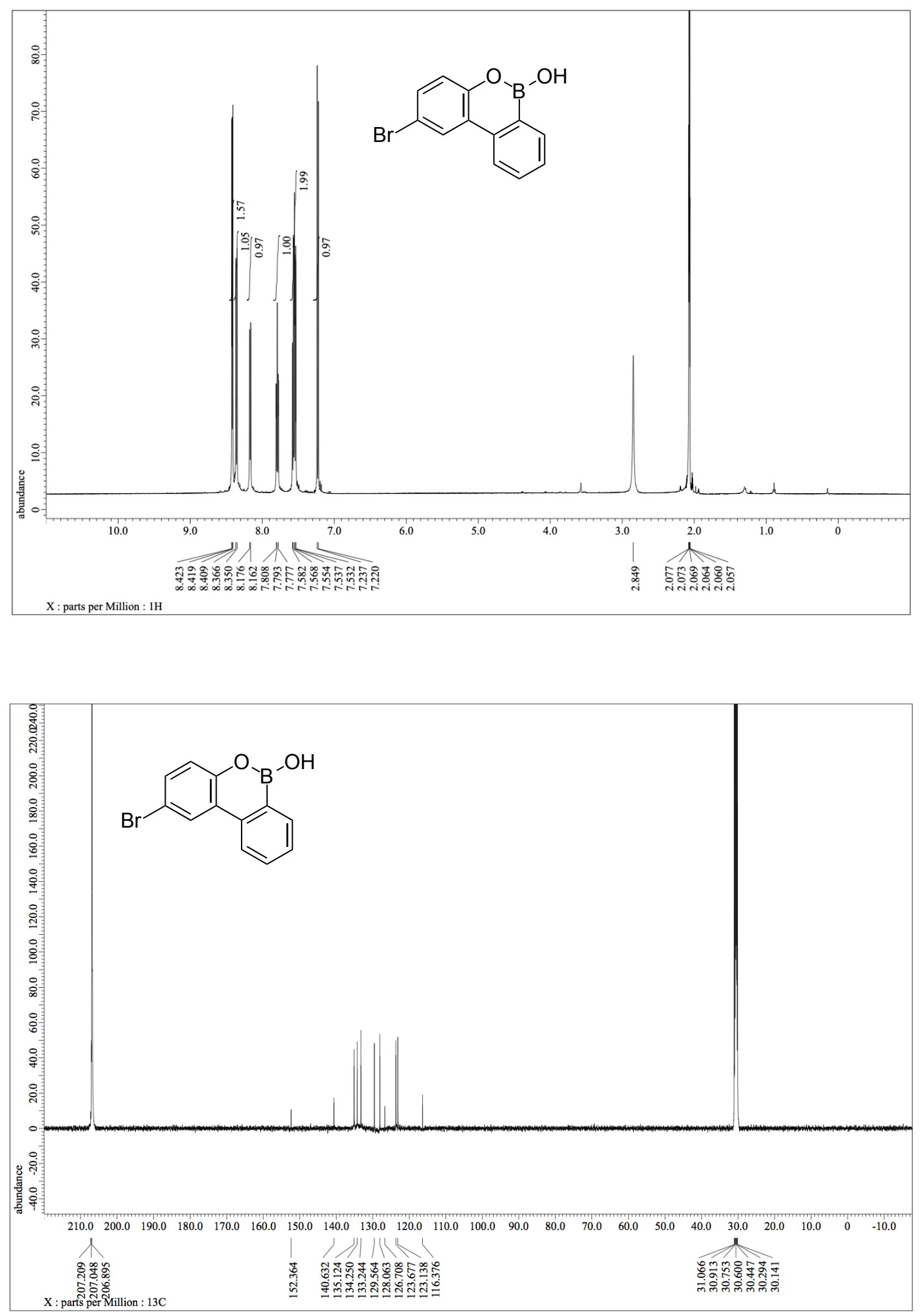
${ }^{1} \mathrm{H}$ NMR $(500 \mathrm{MHz})$ and ${ }^{13} \mathrm{C}$ NMR (126 MHz) spectra of $1 \mathbf{r}$ (acetone- $d_{6}$ )
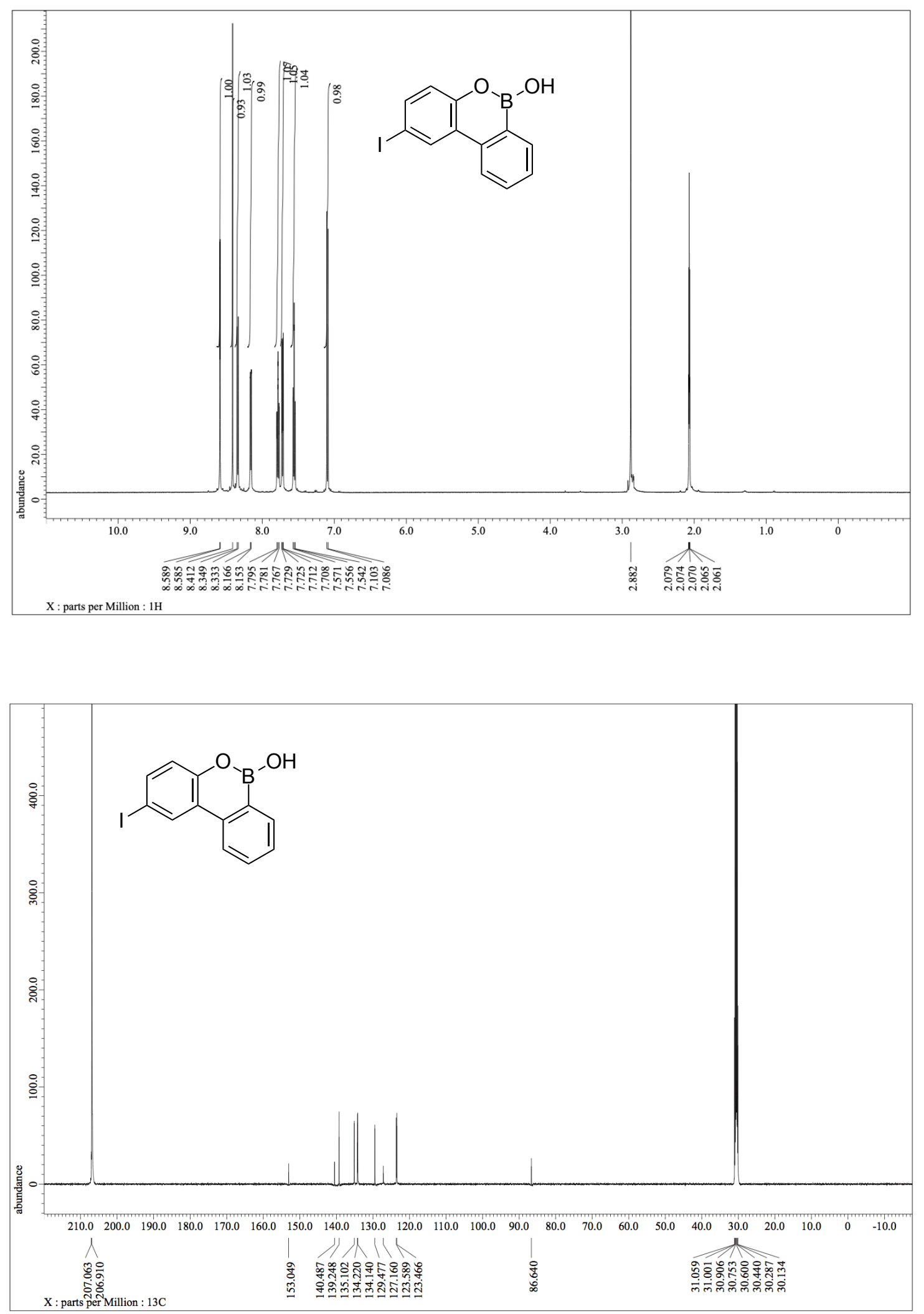
${ }^{1} \mathrm{H}$ NMR (500 MHz) and ${ }^{13} \mathrm{C}$ NMR (126 MHz) spectra of 1 s (acetone- $d_{6}$ )
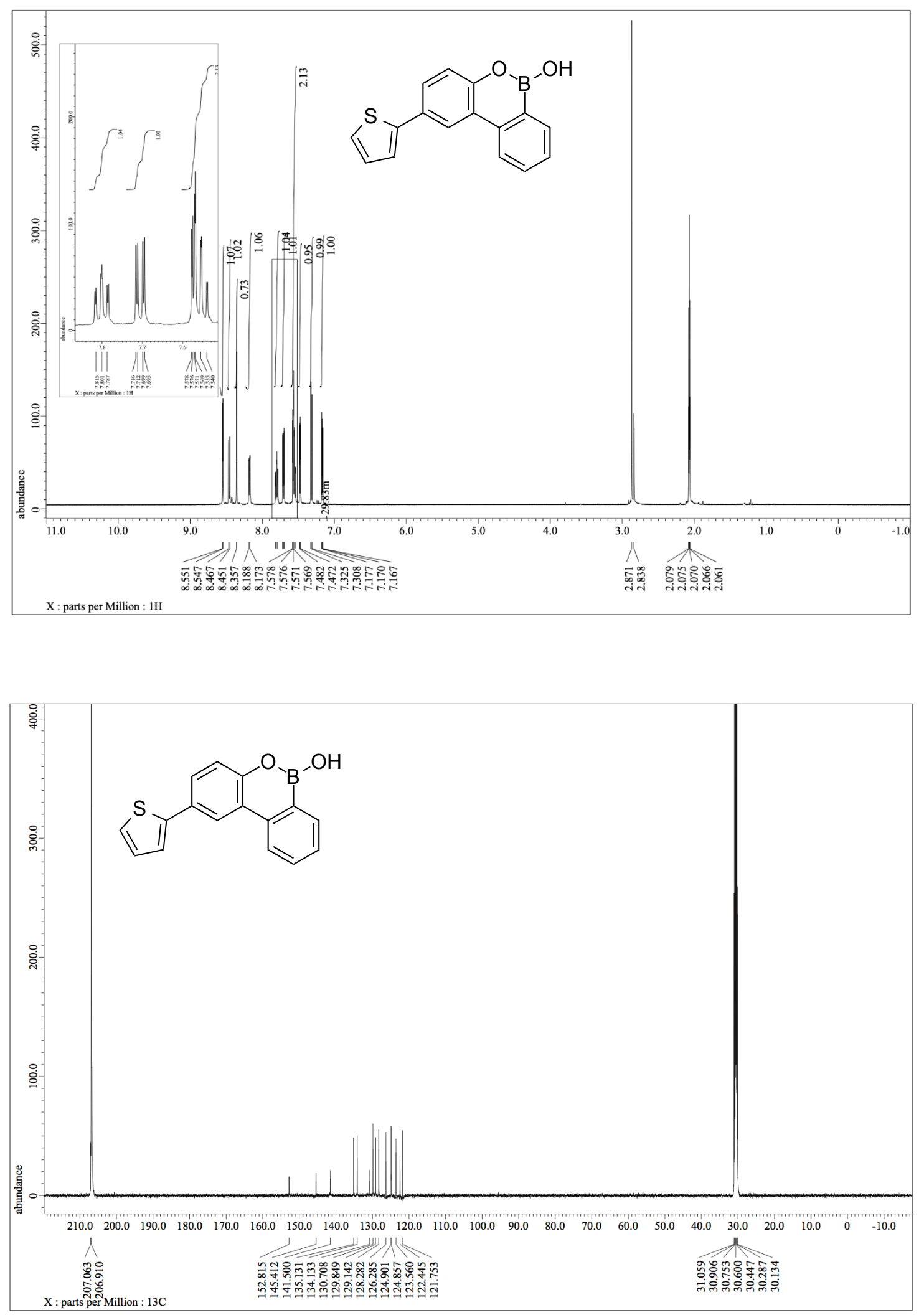
${ }^{1} \mathrm{H}$ NMR $(500 \mathrm{MHz})$ and ${ }^{13} \mathrm{C}$ NMR (126 MHz) spectra of $\mathbf{1 t}$ (acetone- $d_{6}$ )
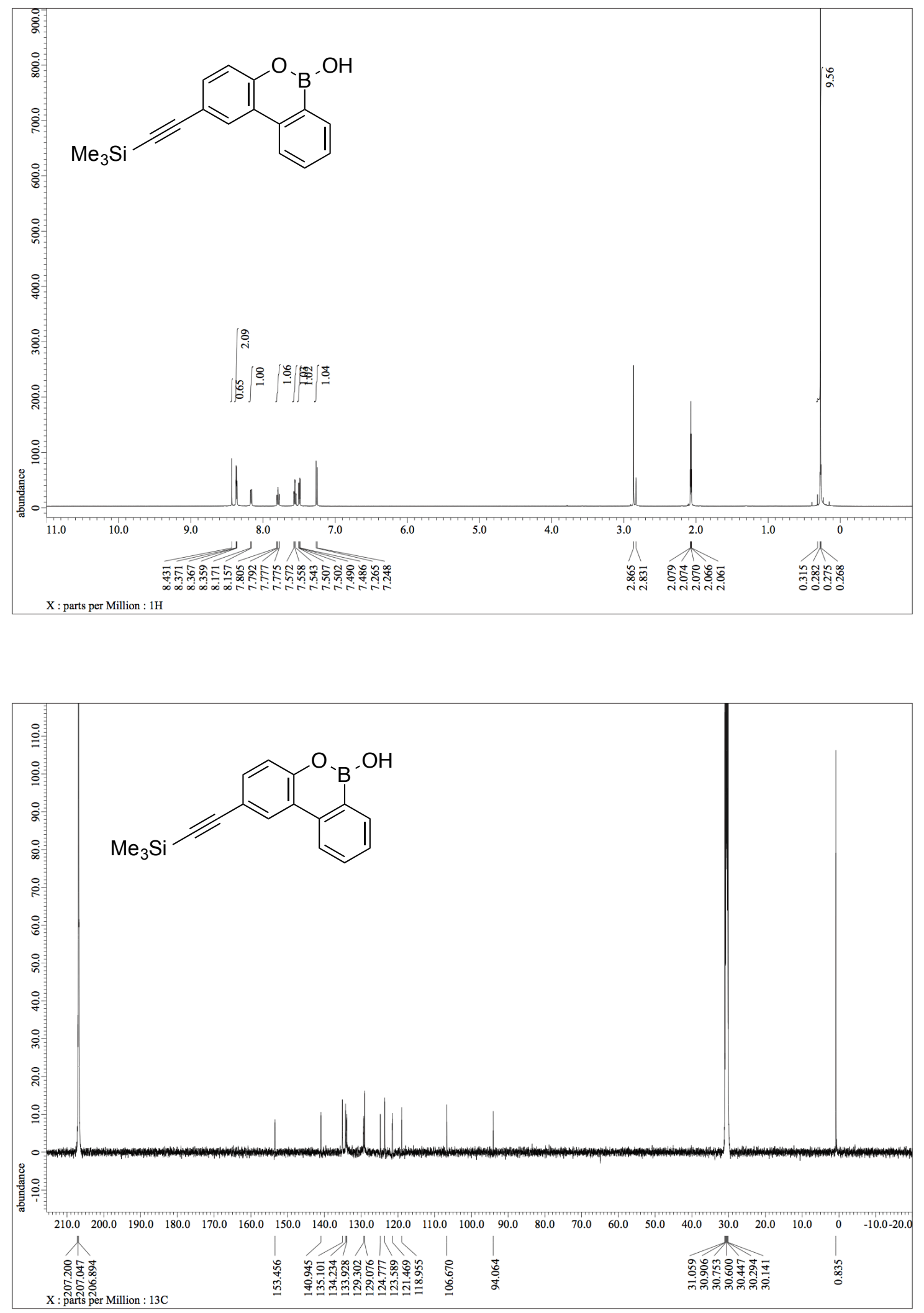
${ }^{1} \mathrm{H}$ NMR $(400 \mathrm{MHz})$ and ${ }^{13} \mathrm{C}$ NMR $(100 \mathrm{MHz})$ spectra of $\mathbf{2 q}\left(\mathrm{CDCl}_{3}\right)$
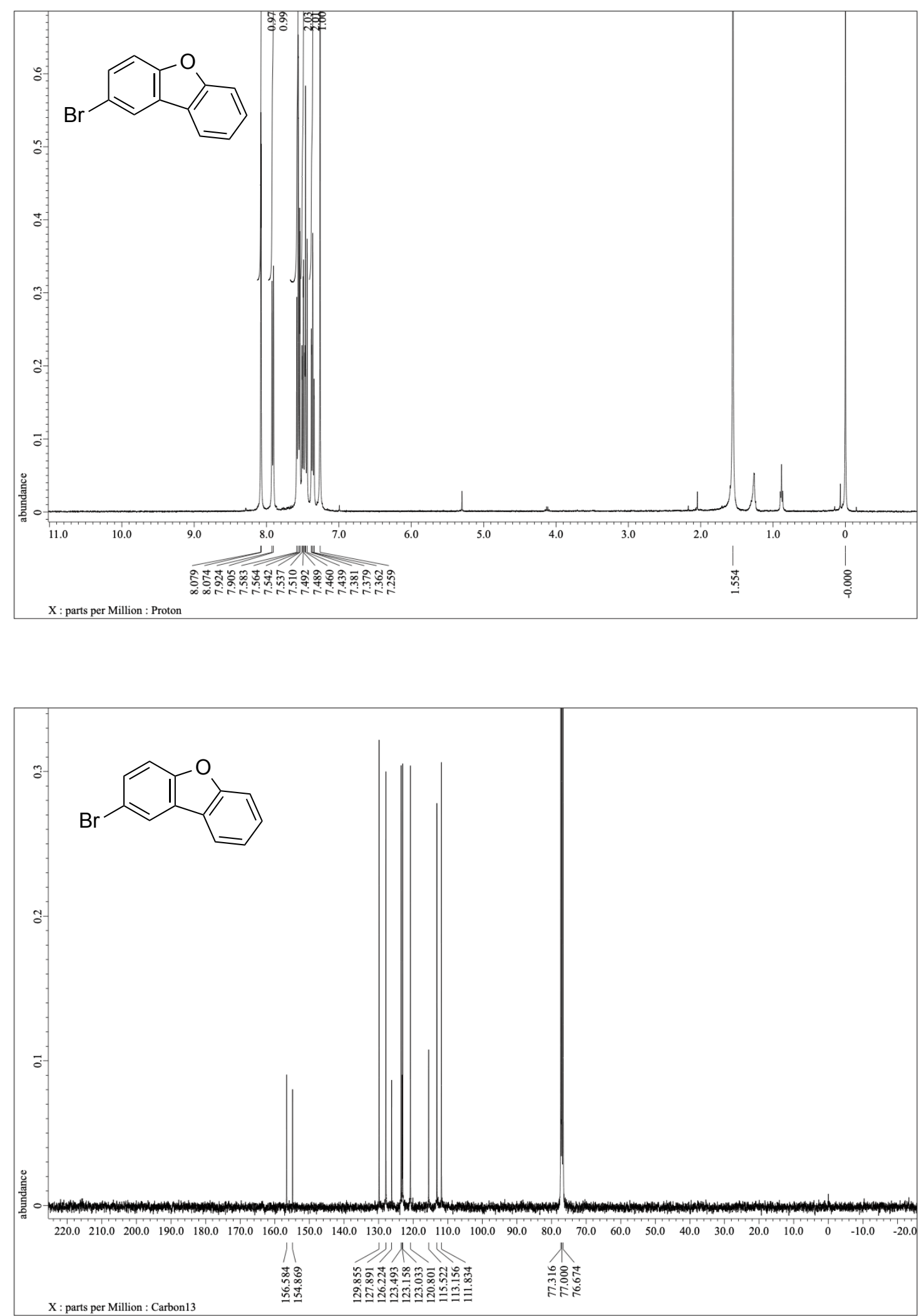
${ }^{1} \mathrm{H}$ NMR $(400 \mathrm{MHz})$ and ${ }^{13} \mathrm{C}$ NMR $(100 \mathrm{MHz})$ spectra of $\mathbf{2 r}\left(\mathrm{CDCl}_{3}\right)$
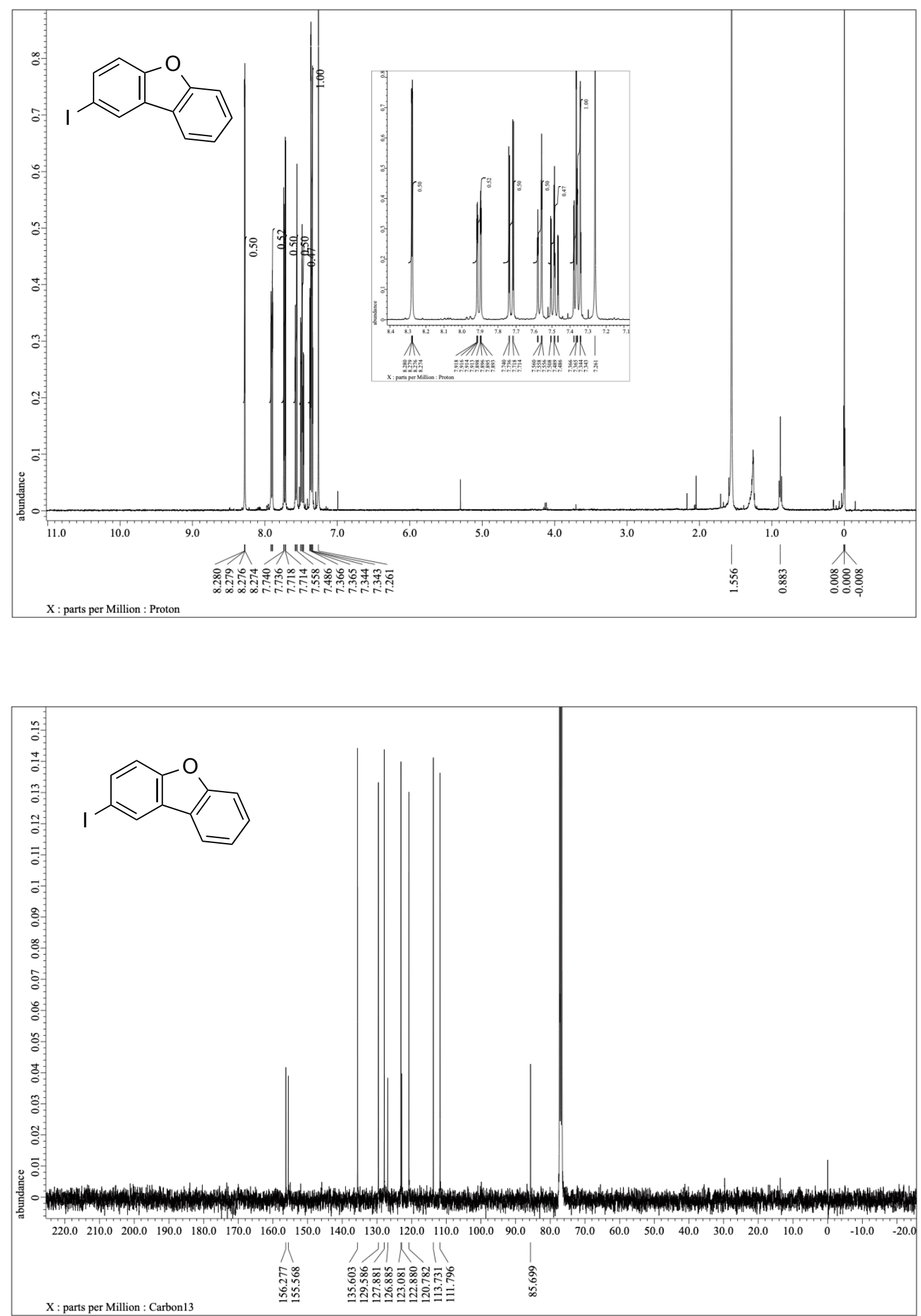
${ }^{1} \mathrm{H}$ NMR $(400 \mathrm{MHz})$ and ${ }^{13} \mathrm{C}$ NMR $(100 \mathrm{MHz})$ spectra of $2 \mathbf{s}\left(\mathrm{CDCl}_{3}\right)$
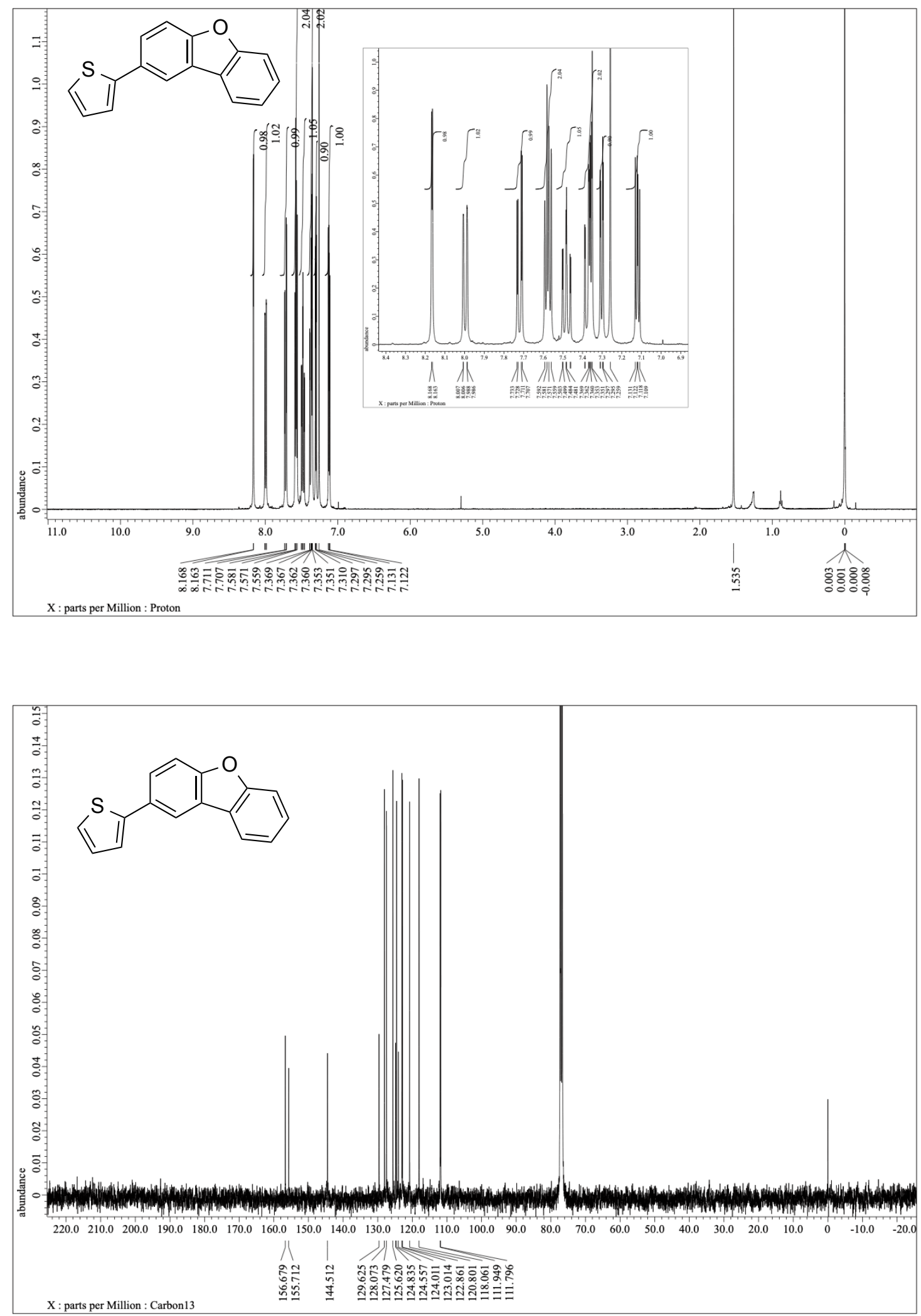
${ }^{1} \mathrm{H}$ NMR $(400 \mathrm{MHz})$ and ${ }^{13} \mathrm{C}$ NMR $(100 \mathrm{MHz})$ spectra of $\mathbf{2 t}\left(\mathrm{CDCl}_{3}\right)$
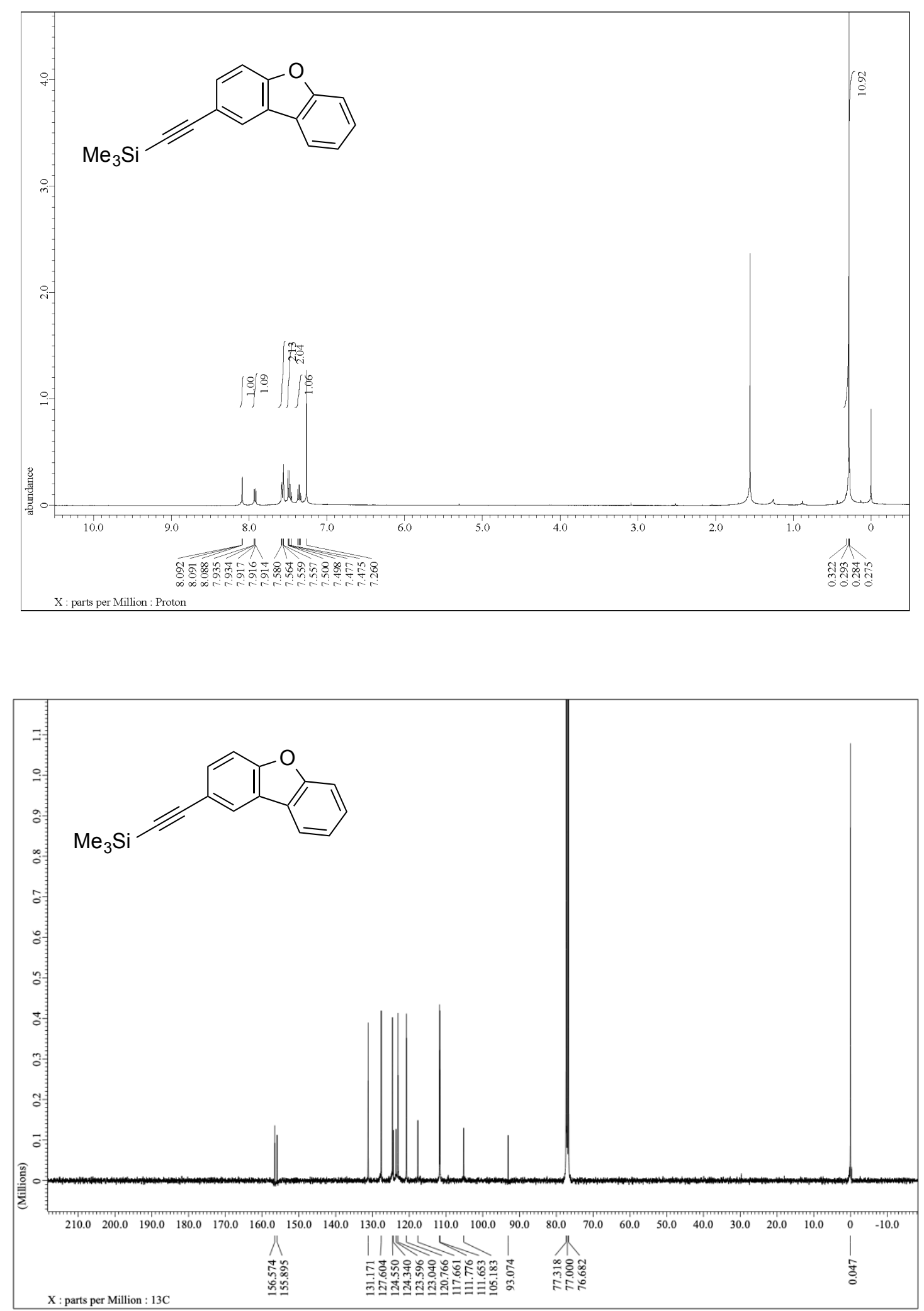
${ }^{1} \mathrm{H}$ NMR (400 MHz), ${ }^{13} \mathrm{C}$ NMR (100 MHz), and ${ }^{19} \mathrm{~F}$ NMR (372.5 MHz) spectra of $7\left(\mathrm{CDCl}_{3}\right)$
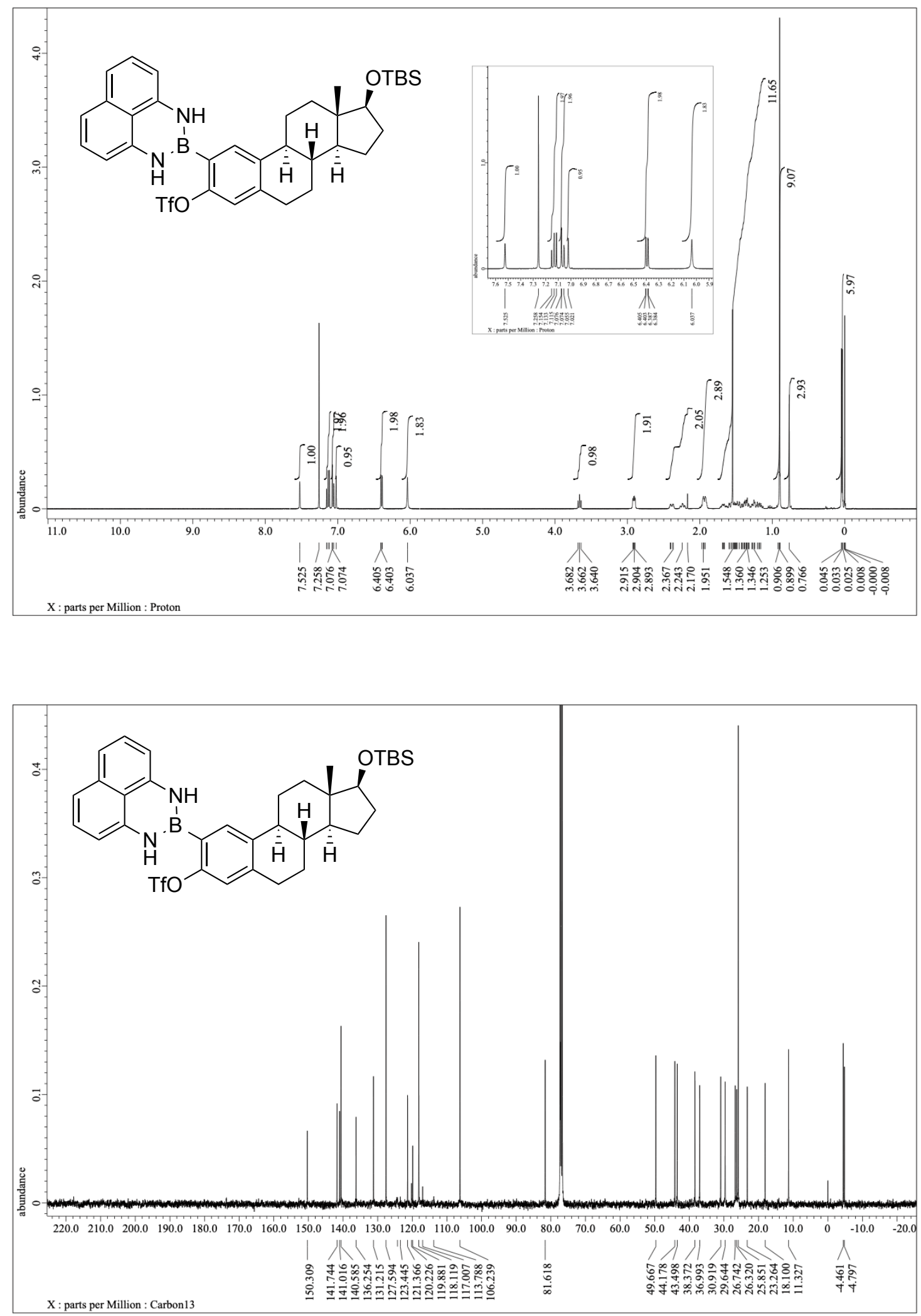


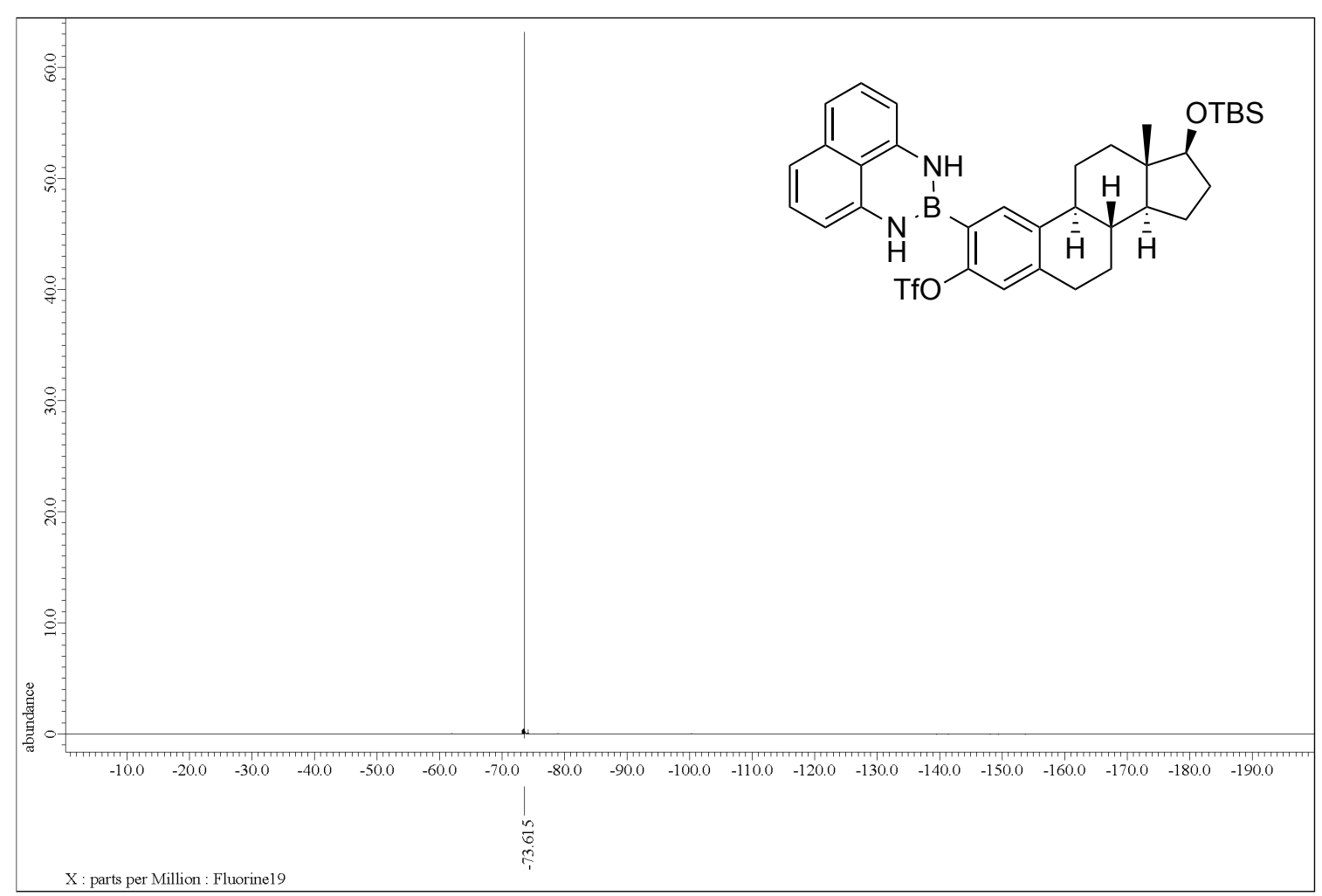


${ }^{1} \mathrm{H}$ NMR $(400 \mathrm{MHz})$ and ${ }^{13} \mathrm{C}$ NMR $(100 \mathrm{MHz})$ spectra of $\mathbf{1} \mathbf{u}\left(\mathrm{CDCl}_{3}\right)$
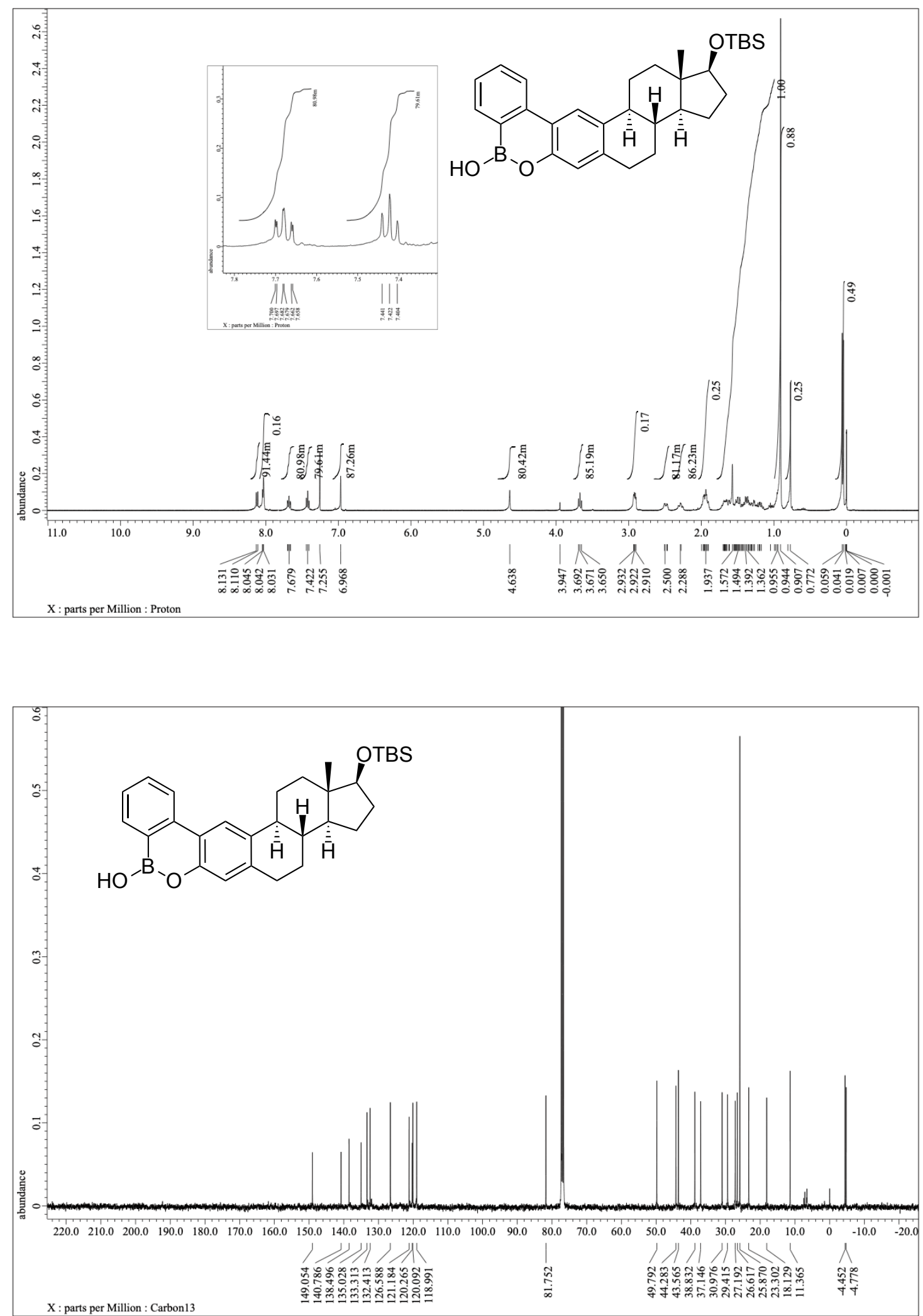
${ }^{1} \mathrm{H}$ NMR $(400 \mathrm{MHz})$ and ${ }^{13} \mathrm{C}$ NMR $(100 \mathrm{MHz})$ spectra of $\mathbf{1 v}\left(\mathrm{CDCl}_{3}\right)$
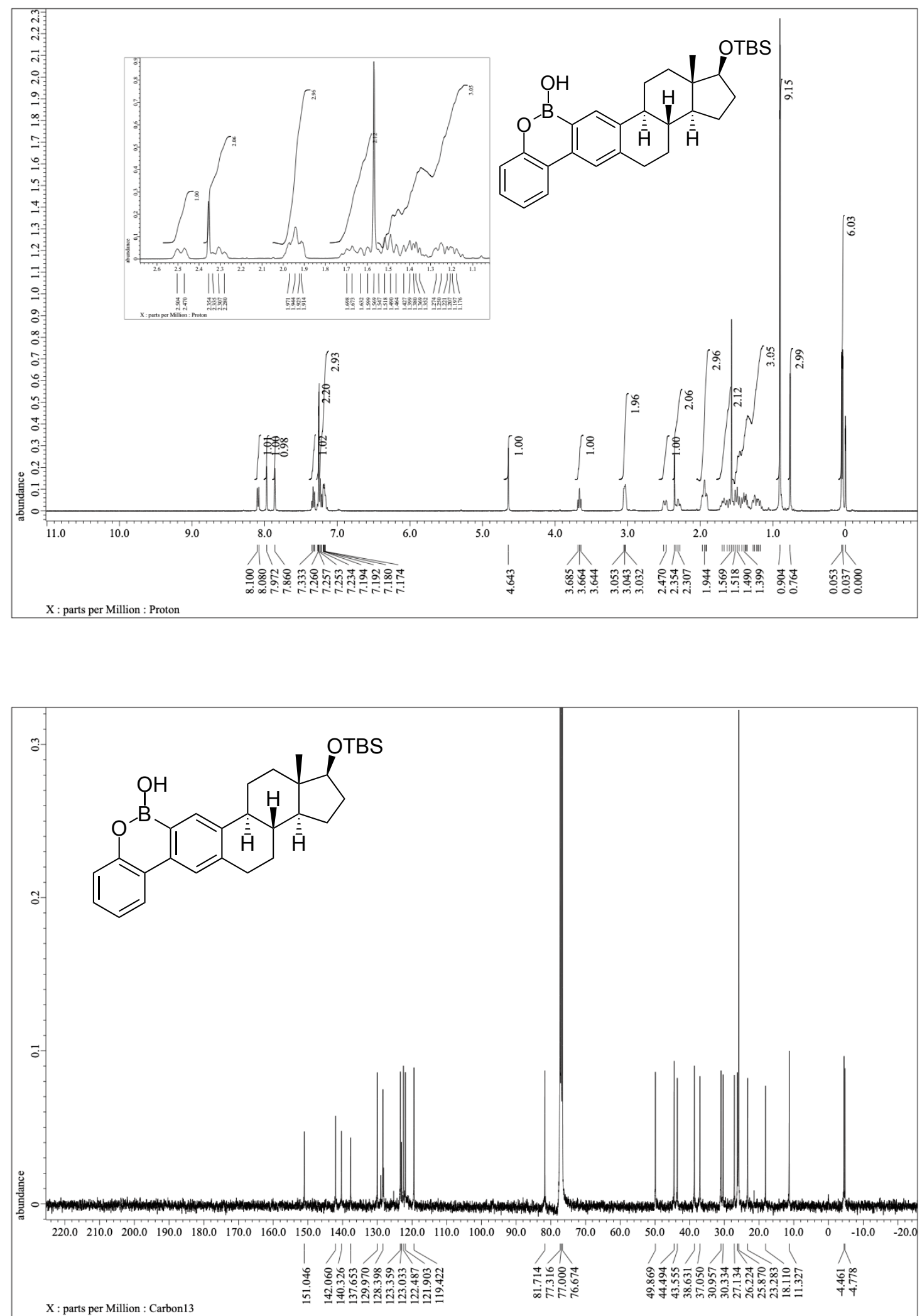
${ }^{1} \mathrm{H}$ NMR $(400 \mathrm{MHz})$ and ${ }^{13} \mathrm{C}$ NMR $(100 \mathrm{MHz})$ spectra of $\mathbf{2} \mathbf{u}\left(\mathrm{CDCl}_{3}\right)$
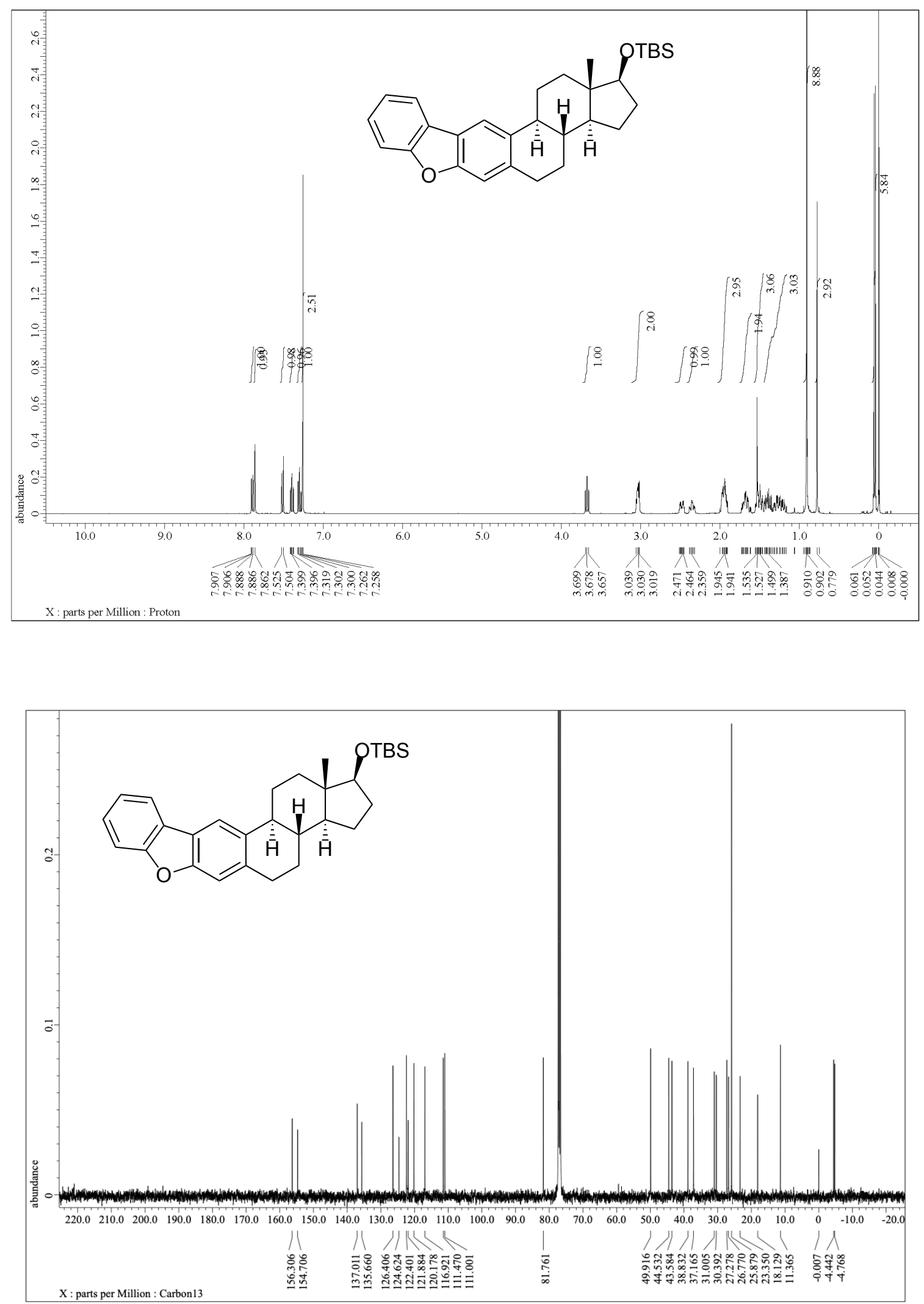
${ }^{1} \mathrm{H}$ NMR $(400 \mathrm{MHz})$ and ${ }^{13} \mathrm{C}$ NMR $(100 \mathrm{MHz})$ spectra of $\mathbf{2 v}\left(\mathrm{CDCl}_{3}\right)$
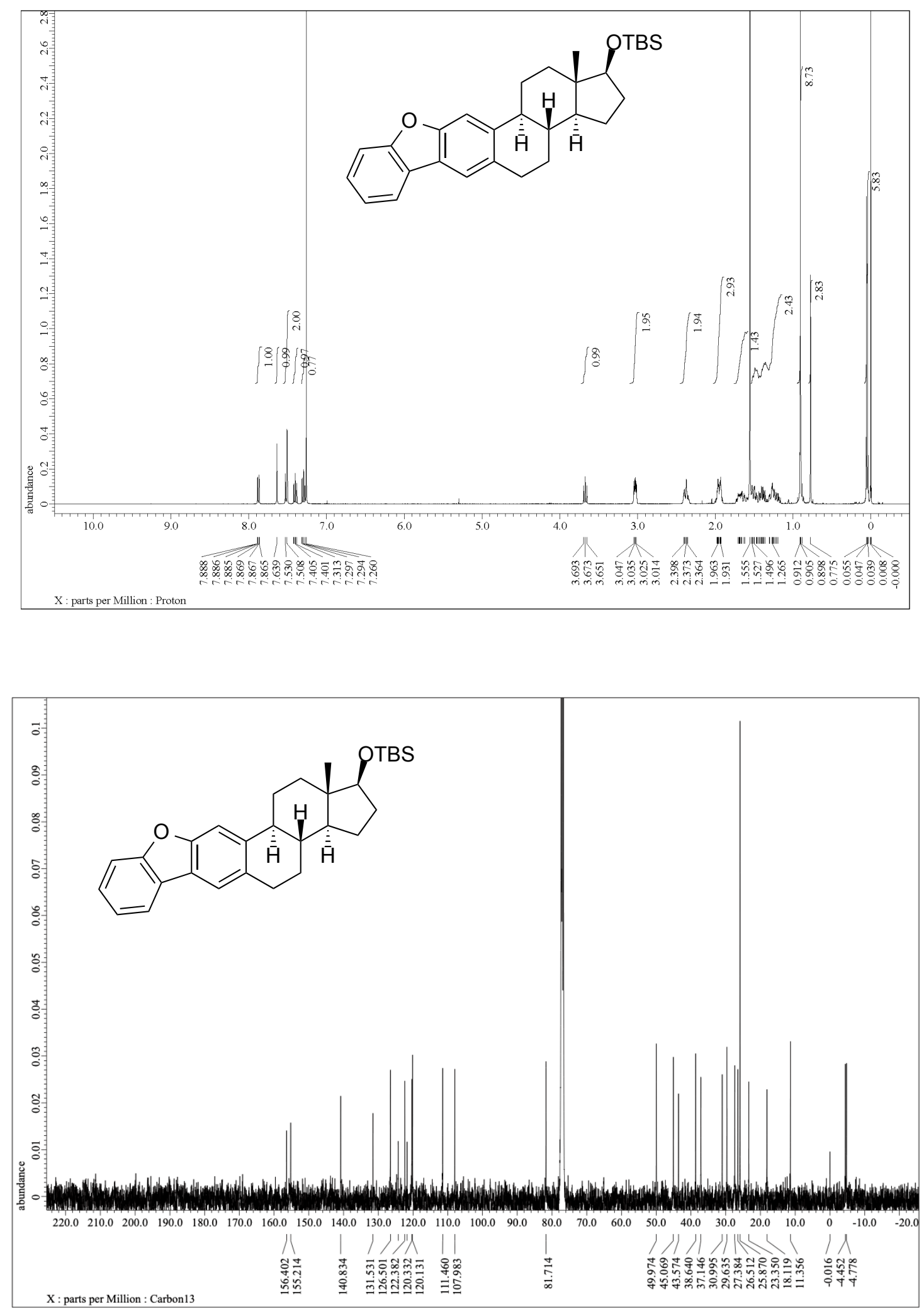